\title{
Behavior Genetics Association 50th Annual Meeting Abstracts
}

\section{Directional relationships between childhood psychopathology dimensions across development}

Andrea G. Allegrini ${ }^{1}$, Toos van Beijsterveldt ${ }^{2}$, Dorret Boomsma ${ }^{2}$, Kaili Rimfeld ${ }^{1}$, Jean-Baptiste Pingault ${ }^{1}$, Robert Plomin ${ }^{1}$, Meike Bartels², Michel Nivard ${ }^{2}$

${ }^{1}$ Social, Genetic and Developmental Psychiatry Centre, Institute of Psychiatry, Psychology and Neuroscience, King's College London, London, United Kingdom

${ }^{2}$ Department of Biological Psychology, Vrije Universiteit Amsterdam, Amsterdam, the Netherlands

Keywords Within-family, Network approach, Child psychopathology, Comorbidity, Sibling interactions

Disentangling trait-like between-person from state-like within-person processes of psychiatric traits across childhood is vital to understand causes of comorbidity, and to gain insights on developmental pathways underlying mental health problems. We present results of a preregistered study conducted in two large population-based cohorts, the Twin Early Developmental Study and the Netherlands Twin Register, where we investigated the longitudinal directional relationships between psychopathology-related traits from childhood to early adolescence, jointly estimating between-person and withinperson processes within a network model.

The contrast between trait-like individual differences and statelike within-person processes can be extended to the family. Members of a family, especially siblings, are known to behave alike and this can be attributed to shared genetic and environmental influences. However, there are obvious direct interactions between siblings, agespecific symptoms in one sibling, which could precipitate mental symptoms in the other sibling at a later age.

We developed an extension of the network model to family-level data by considering sibling pairs instead of unrelated individuals. Aim of this extension is to estimate reciprocal directional influences between siblings over time separating them from similarities between siblings that arise through shared (genetic or environmental) influences that exist in a family. Furthermore, this application can be used to parse out genetic and environmental components of variance at the level of time-invariant overarching stable traits, as well as agespecific effects. In practice, our approach takes the network model from an individual to a family level, while controlling for the fact that family members are related to each other.
Grant Support: This project has received funding from the European Union's Horizon 2020 research and innovation programme under the Marie Sklodowska-curie Grant Agreement No. 721567.

\section{Genetics of autistic traits in the general population reveals potential role for immune-related genes in autism spectrum disorder}

M. Arenelli ${ }^{1}$, G. Cadby ${ }^{2}$, W. de Witte ${ }^{1}$, R.M. Jones ${ }^{2}$, A.J.O. Whitehouse ${ }^{4}$, E.K. Moses ${ }^{2,5}$, A. Fornito ${ }^{6}$, M.A. Bellgrove ${ }^{6}$, J.K. Buitelaar ${ }^{7,8,9}$, L.A. Kiemeney ${ }^{10}$, G. Poelmans ${ }^{1}$, J. Bralten ${ }^{1,7}$

${ }^{1}$ Department of Human Genetics, Radboud University Medical Center Nijmegen, The Netherlands

${ }^{2}$ Centre for Genetic Origins of Health and Disease, School of Biomedical Sciences, University of Western Australia, Crawley, Australia

${ }^{3}$ School of Population and Global Health, University of Western Australia, Crawley, Australia

${ }^{4}$ Kids Institute, University of Western Australia, Perth, Australia

${ }^{5}$ Menzies Institute for Medical Research, University of Tasmania, Hobart, Australia

${ }^{6}$ Monash Clinical and Imaging Neuroscience, School of Psychological Science \& Turner Institute for Brain and Mental Health and Monash Biomedical Imaging, Monash University, Clayton, Victoria, Australia

${ }^{7}$ Donders Institute for Brain, Cognition and Behaviour, Radboud University, Nijmegen, The Netherlands

${ }^{8}$ Karakter Child and Adolescent Psychiatry University Centre, Nijmegen, The Netherlands

${ }^{9}$ Department of Cognitive Neuroscience, Radboud University Medical Center, Nijmegen, The Netherlands

${ }^{10}$ Radboud Institute for Health Sciences, Radboud University Medical Center, Nijmegen, The Netherlands

Keywords Autism spectrum disorder; Autistic-like traits; Immune system; Synaptic signalling

Autism spectrum disorders (ASDs), common complex and highly heritable neurodevelopmental disorders (Tick et al. 2016), represent the extreme manifestation of autistic-like traits (ALTs) measurable in the general population (Robinson et al. 2016). ASDs and ALTs are 
genetically correlated meaning that common genetic variants for ALTs also play a role in the clinical condition of ASDs (Bralten et al. 2018). In this regard, ALTs may constitute a more accessible and potentially less heterogeneous route to the identification of common risk variants for ASDs.

This study meta-analysed genome-wide association results for four ALTs-'attention-to-detail', 'imagination', 'rigidity', and 'socialskills' - of 4600 healthy individuals. The existence of a shared genetic aetiology between each ALT and ASDs was tested using summary statistics from the Psychiatric Genomic Consortium of ASDs $(n=46,350)$. Gene-wide and gene-set analyses were used to prioritize genes and investigate the underlying biology of the four ALTs independently. Gene co-expression analyses were performed to highlight enrichment of biological processes across a range of ASDrelated brain regions.

We found two novel genome-wide significant loci associated with 'attention-to-detail' (rs6125844, rs3731197). Significant shared genetic aetiology was observed between ASDs and 'rigidity'. A lookup of the top variants along with gene-wide analyses highlighted immune-related genes in ALTs, namely RNF114, CDKN2A, KAZN, SPATA2, and ZNF816A. Following gene co-expression network analyses confirmed a role of genes involved in the immune response, together with neuronal and synaptic signalling, differentially expressed ASD-related brain regions for multiple ALTs.

This study offers novel insights into the biology of autistic-like features and suggests a role of immune regulation in 'attention-todetail' and 'rigidity'. Also, our results confirm that ASDs and ALTs share genetic loci and processes and thereby encourage the use of population-based quantitative ALTs to address the complex genetics of ASDs.

\section{References}

Tick, B., Bolton, P., Happé, F., Rutter, M., \& Rijsdijk, F. (2016). Heritability of autism spectrum disorders: a meta-analysis of twin studies. Journal of Child Psychology and Psychiatry, 57(5), 585-595. Robinson, E. B., St Pourcain, B., Anttila, V., Kosmicki, J. A., BulikSullivan, B., Grove, J., .. \& M Martin, J. (2016). Genetic risk for autism spectrum disorders and neuropsychiatric variation in the general population. Nature genetics, 48(5), 552.

Bralten, J., Van Hulzen, K. J., Martens, M. B., Galesloot, T. E., Vasquez, A. A., Kiemeney, L. A., ... \& Poelmans, G. (2018). Autism spectrum disorders and autistic traits share genetics and biology. Molecular psychiatry, 23(5), 1205-1212.

\section{Two genetic analyses to elucidate causality in the associations between body mass index and psychological traits}

Kadri Arumäe ${ }^{1}$, Daniel Briley ${ }^{2}$, Lucía Colodro-Conde ${ }^{3}$, Erik Lykke Mortensen ${ }^{4}$, Kerry Jang ${ }^{5}$, Juko Ando ${ }^{6}$, Christian Kandler ${ }^{7}$, Thorkild I. A. Sørensen ${ }^{4}$, Alain Dagher ${ }^{8}$, René Mõttus ${ }^{9,1}$, Uku Vainik ${ }^{1,8}$

${ }^{1}$ Institute of Psychology, University of Tartu, Tartu, Estonia

${ }^{2}$ Department of Psychology, The University of Illinois at UrbanaChampaign, IL, USA

${ }^{3}$ QIMR Berghofer Medical Research Institute, Brisbane, Australia

${ }^{4}$ Department of Public Health, University of Copenhagen, Copenhagen, Denmark

${ }^{5}$ Division of Behavioural Sciences, Department of Psychiatry, The University of British Columbia, Vancouver, Canada

${ }^{6}$ Faculty of Letters, Keio University, Tokyo, Japan

${ }^{7}$ Department of Psychology, University of Bremen, Germany
${ }^{8}$ Montreal Neurological Institute, McGill University, Montreal, Canada

${ }^{9}$ Department of Psychology, University of Edinburgh, United Kingdom

Keywords Direction of causation, Allele Score, Personality, Mendelian randomization, Nuances

Many personality traits correlate with BMI, but the existence and direction of causal links between them have are unclear. If personality influences BMI, knowing causal direction could inform weight management strategies. If BMI instead influences personality, knowing this would contribute to a better understanding of the effects of weight change and the mechanisms of personality development.

We employed two genetically informed methods. In Mendelian randomization ( $\mathrm{N}=3541$ from Estonian Biobank), allele scores for personality traits Neuroticism, Worry, and Depressive Affect were set to predict BMI. Similarly, allele score for BMI was used to predict eating-specific and domain-general phenotypic personality traits. PPS-s are aggregate scores of NEO PI-R personality traits weighted by BMI. In Direction of Causation, twin data from five countries ( $\mathrm{N}=$ 5424) were used to assess the fit of alternative causal models: PPSs influencing BMI, BMI influencing PPSs, reciprocal causation, and no causation.

In Mendelian randomization, the allele score for BMI predicted domain-general $(\beta=0.04, P=.022)$ and eating-specific PPS-s $(\beta=$ $0.04, P=.012)$. In reverse, only the allele score for Worry predicted BMI ( $\beta=-0.07, P<.001)$. In Direction of Causation, BMI similarly predicted domain-general $(\beta=0.21, P<.001)$ and eating-specific PPS-s $(\beta=0.19, P<.001)$. In exploratory analyses, causal links between BMI and domain-general personality traits appeared reciprocal for higher-weight individuals (BMI $>\sim 25$ ).

Results suggest an influence of BMI on personality; influences of personality on BMI appear limited. Centering weight management interventions around personality may therefore not provide additional benefits, but maintaining or achieving normal-range weight may contribute to a more favorable personality profile ( https://osf.io/preprints/nutrixiv/q8ehr/).

Grant Support: Uku Vainik is supported by Estonian Research Council's Grant MOBTP94.

\section{Early manifestations of intellectual ability: Evidence that genetic effects on achievement are mediated through early childhood literacy}

Chloe Austerberry ${ }^{1}$, Pasco Fearon ${ }^{1}$, Angelica Ronald ${ }^{2}$, Leslie D. Leve $^{3}$, Jody M. Ganiban ${ }^{4}$, Misaki N. Natsuaki ${ }^{5}$, David Reiss ${ }^{6}$, Daniel S. Shaw ${ }^{7}$, Jenae M. Neiderhiser ${ }^{8}$

${ }^{1}$ Department of Clinical, Educational and Health Psychology, UCL, UK

${ }^{2}$ Department of Psychological Sciences, Birkbeck, University of London, UK

${ }^{3}$ Prevention Science Institute, University of Oregon, Eugene, OR, USA

${ }^{4}$ Department of Psychology, George Washington University, Washington, DC, USA

${ }^{5}$ Department of Psychology, University of California, Riverside, CA, USA

${ }^{6}$ Yale Child Study Center, Yale School of Medicine, New Haven, CT, USA

${ }^{7}$ Department of Psychology, University of Pittsburgh, Pittsburgh, PA, USA 
${ }^{8}$ Department of Psychology, The Pennsylvania State University, University Park, PA, USA

Keywords Literacy; Executive function; Achievement; Adoption; Genetic

Intellectual ability is one of the strongest predictors of lifelong success, health and longevity. Achievement in middle childhood is reliably linked to intellectual ability in adulthood and there is convincing evidence that genetic mechanisms underly this association. However, earlier indicators of intellectual ability are less well understood. While executive function (EF) and literacy are promising early markers, it remains unclear whether these abilities in infancy and early childhood have shared pathways with the development of later achievement or simply predict these outcomes. We used the powerful adoption design to examine the role of genetic factors, early $\mathrm{EF}$ and literacy in the development of later achievement, in 561 linked sets of adopted children and their birth and adoptive parents. Latent measures of birth parent intellectual ability were used as indicators of genetic influence on children's intellectual outcomes, and multiple observational measures and tests were used to index child EF, literacy, and achievement. We hypothesized that birth parent intellectual ability would predict child EF, literacy and achievement, and that early childhood EF and literacy would mediate the association between birth parent intellectual ability and child achievement at 7 years. The results suggested that genetic factors underlying birth parent intellectual ability may influence EF at 27 months but not later in childhood, literacy from 4.5 years, and achievement at 7 years. There was no evidence that genetic effects on achievement were mediated through EF at 27 months. However, literacy at 4.5 years mediated the effects of genetic influences on achievement at 7 years.

Grant Support: This project was supported by Grant R01 HD042608 from the Eunice Kennedy Shriver National Institute of Child Health and Human Development and the National Institute on Drug Abuse, NIH, U.S. PHS (PI Years 1-5: David Reiss, MD; PI Years 6-10: Leslie Leve, PhD), and by grant R01 DA020585 from the National Institute on Drug Abuse, the National Institute of Mental Health and OBSSR, NIH, U.S. PHS (PI: Jenae Neiderhiser, PhD). Chloe Austerberry's contribution to this work was supported by her ESRC funded, UBEL-DTP studentship.

\section{The nature of nurture revisited: estimating the effect of vertical transmission using structural equation modeling}

Jared V. Balbona ${ }^{1,2}$, Yongkang Kim ${ }^{1}$, and Matthew C. Keller ${ }^{1,2}$ ${ }^{1}$ Institute of Behavioral Genetics, University of Colorado Boulder, Boulder, Colorado, USA

${ }^{2}$ Department of Psychology and Neuroscience, University of Colorado Boulder, Boulder, Colorado, USA

Keywords Vertical transmission, Genetic nurture, Heritability estimation, Structural equation modeling

Although the role of parental effects-both genetic and non-geneticon offspring phenotypes has long been a primary focus of behavioral genetics research, family studies have struggled to disentangle variance due to shared genetic factors from variance due to shared environmental factors. As a result, much remains unknown about the extent to which parental phenotypes directly influence offspring phenotypes (a process known as "vertical transmission"). A recent paper by Kong et al. (2018) sought to address this gap by examining the effect of parents' non-transmitted alleles on their child's phenotypes. Here, we introduce a path analysis of Kong et al.'s basic idea that (1) Builds on Kong et al. (2018) by properly accounting for assortative mating at equilibrium; (2) Provides an unbiased estimate of phenotypic variance due to vertical transmission, even when a trait's polygenic risk score accounts for only a small fraction of total genetic variance; (3) Easily allows for extensions of the basic model, such as modeling phenotypic covariance between relative types and allowing unequal familial environmental effects between mothers and fathers. In utilizing principles of structural equation modeling, this method greatly simplifies the math used by Kong et al. (2018) and allows for direct comparisons between our model and other classic family models used in behavioral genetics research.

Grant Support: NIH R01 MH100141 (Keller).

\section{Investigating the specificity of the genetic associations between smoking behaviours and psychiatric traits and disorders with genomic structural equation modelling}

Wikus Barkhuizen $^{1,2}$; Frank Dudbridge ${ }^{3}$ and Angelica Ronald ${ }^{1}$ ${ }^{1}$ Centre for Brain and Cognitive Development, Department of Psychological Sciences, Birkbeck, University of London, UK

${ }^{2}$ Department of Clinical, Educational and Health Psychology, University College London, London, UK

${ }^{3}$ Department of Health Sciences, University of Leicester, UK

Keywords Psychotic experiences; Schizophrenia; Depression; Bipolar disorder; Genomic SEM

The phenotypic association between cigarette smoking and major psychiatric disorders is well established. Psychiatric traits in the general population such as psychotic experiences are also associated with smoking, even after accounting for co-occurring risks such as cannabis and alcohol use. We aimed to investigate whether genetic overlap between smoking and psychiatric traits and disorders exists beyond genetic influences shared with confounding variables.

Bivariate genetic correlations between smoking behaviours (cigarettes per day, smoking status, age of smoking initiation and smoking cessation) and psychiatric disorders (schizophrenia, major depression and bipolar disorder) and with psychotic experiences in adolescents and adults were computed in LD score regression. Fully saturated genomic multiple regression models were specified in Genomic SEM to estimate genetic associations between smoking and psychiatric traits and disorders conditional on genome-wide genetic influences on confounders (cannabis and alcohol use, risk taking and insomnia).

Significant bivariate genetic correlations were found between smoking and psychotic experiences in adulthood $\left(\mathrm{r}_{\mathrm{g}}=.33-.40\right)$ as well as between smoking and psychiatric disorders $\left(\mathrm{r}_{\mathrm{g}}=-.08-.38\right)$. Genomic SEM indicated that genetic associations (conditional on covarying genetic influences on the confounders) remained between most smoking behaviours and schizophrenia and depression $\left(\mathrm{b}_{\mathrm{g}}=\right.$ $.14-40$ ) but not between smoking and bipolar disorder or most psychotic experiences.

Our results suggest that pleiotropy between cigarette smoking and some psychiatric disorders likely reflect shared biological pathways whereas pleiotropy between smoking and psychotic experiences may be attributable to genetic influences of co-occurring risk factors.

Grant Support: This work was supported by the UK Medical Research Council (G1100559 to AR) and a Wellcome Trust ISSF grant (204770/Z/16/Z) and the Camara-Rijvers David Studentship to WB. 


\section{Genetic influence on Secure Attachment Depends on Socioeconomic Status}

Christopher R Beam ${ }^{1,2,3}$, Deborah Finkel ${ }^{4,5}$, Evan J. Giangrande ${ }^{6}$, Alyssa Morris ${ }^{1}$, Deborah W. Davis ${ }^{7}$

${ }^{1}$ Department of Psychology, University of Southern California, Los Angeles, California, USA

${ }^{2}$ School of Gerontology, University of Southern California, Los Angeles, California, USA

${ }^{3}$ Center for the Changing Family, University of Southern California, Los Angeles, California, USA

${ }^{4}$ Department of Psychology, Indiana University Southeast, New Albany, Indiana, USA

${ }^{5}$ Institute for Gerontology, Jönköping University, Småland, Sweden

${ }^{6}$ Department of Psychology, University of Virginia, Charlottesville, Virginia, USA

${ }^{7}$ Department of Pediatrics, University of Louisville, Louisville, Kentucky, USA

Keywords Attachment, SES, Gene-environment interaction, Louisville twin study

Attachment behavioral systems are formed in childhood, persist over the lifespan, and correlate with socioeconomic status (SES; van Ijzendoorn and Bakermans-Kranenburg 2013). While attachment can be attributed, in part, to genetic causes of variation (Matheny and Finkel 2000), no twin studies have considered whether SES moderates heritability of attachment systems. Understanding how attachment heritability is moderated by SES may provide insight in maximizing children's genetic potential for secure attachment. We predicted greater heritability in children from high SES families than in low SES families, as would be predicted by social enhancement models. Data were drawn from the Louisville Twin Study and included 161 pairs of 19 - and 25-month-old twin pairs $(\mathrm{MZ}=71, \mathrm{DZ}$ =90). Trained observers coded infants' attachment behavior (secure vs. insecure) in a modified Strange Situation paradigm. SES scores were created using head of household occupation (range: 0-100) with higher scores indicating higher job prestige. Gene-environment interaction models were fit to the data to test differences in heritability of attachment style across SES. Heritability of attachment was greater in children from high SES families than low SES families. Results suggest that improvement in SES may increase children's genetic expression of their attachment styles.

\section{References}

Finkel D \& Matheny AP. (2000). Genetic and environmental influences on a measure of infant attachment security. Twin Res, 0, $242-250$.

van Ijzendoorn MH \& Bakermans-Kranenburg MJ. (2010). Invariance of adult attachment across gender, age, culture, and socioeconomic status? J Soc Pers Rel, 27(2), 200-208.

Grant Support: Funding was supported by National Institute on Aging (US) Grant No. R03AG048850-01 and National Institute on Aging Grant No. R01 AG063949-01.

\section{On the etiology of the intensity of visual aesthetic appraisal and preferences}

Giacomo Bignardi ${ }^{1}$, Dirk JA Smit ${ }^{2}$, Luca F Ticini ${ }^{3}$, MacKenzie D Trupp $^{4}$ and Tinca JC Polderman ${ }^{5,6}$

${ }^{1}$ Laboratory of Neurobiology, Department of Cell and Developmental Biology, University College London, UK
${ }^{2}$ Department of Psychiatry, Amsterdam University Medical Centers, University of Amsterdam, Amsterdam Neuroscience, Amsterdam, The Netherlands

${ }^{3}$ Division of Neuroscience and Experimental Psychology, School of Biological Sciences, The University of Manchester, UK

${ }^{4}$ Faculty of Psychology, Department of Cognition, Emotion, and Methods in Psychology, University of Vienna, Vienna, Austria

${ }^{5}$ Complex Trait Genetics, Center for Neuroscience and Cognitive Research, Vrije Universiteit Amsterdam, Amsterdam, The Netherlands

${ }^{6}$ Department of Child and Adolescent Psychiatry, Amsterdam UMC, Amsterdam, The Netherlands

Keywords Aesthetic, Hedonic, Twin, Faces, Genetic

Visual aesthetic appraisal is a shared yet diversified human experience. While the process of how and why certain visual stimuli acquire value has been the focus of recent extensive research, the contribution of genetic and environmental factors to variation has rarely been examined. Here, we analyzed freely available data from the Australian Twin Registry obtained by Germine et al. (2015, N 1115 identical and 432 fraternal twins), and Sutherland et al. (Sutherland et al. 2020, N 825 identical and 418 fraternal twins) on the variation of perceived attractiveness and pleasantness for faces, sceneries, and abstract images.

We first examined at the phenotypic level to what extent evaluations were shared or unique for each set of stimuli. We then partitioned the amount of unique variance into the individual intensity and preference of visual aesthetic appraisal. Finally, we estimated twin correlations and the contribution of genetic and environmental influences to variation in the three stimuli types.

At the phenotypic level we found that intensity of appraisal for faces, sceneries, and abstract images were moderately correlated across stimuli, while only weakly correlated for preferences. Finally, we found that genetic factors explain part of the variance, $\sim 30 \%$ for the intensity of appraisal and $\sim 20 \%$ for preferences, in all stimuli types and traits except individual preferences for abstract images.

Thus, our study shows that two aspects of human aesthetic evaluation, namely intensity of appraisal and preferences, can be quantified and that, as for several other psychological traits, genetic influences contribute to variation.

\section{References}

Germine, L., Russell, R., Bronstad, P. M., Blokland, G. A. M., Smoller, J. W., Kwok, H., Anthony, S. E., Nakayama, K., Rhodes, G., \& Wilmer, J. B. (2015). Individual Aesthetic Preferences for Faces Are Shaped Mostly by Environments, Not Genes. Current Biology: $C B, 25(20), 2684-2689$. https://doi.org/10.1016/j.cub.2015.08.048

Sutherland, C. A. M., Burton, N. S., Wilmer, J. B., Blokland, G. A. M., Germine, L., Palermo, R., Collova, J. R., \& Rhodes, G. (2020). Individual differences in trust evaluations are shaped mostly by environments, not genes. Proceedings of the National Academy of Sciences, 117(19), 10218-10224. https://doi.org/10.10 73/pnas.1920131117

\section{Alcohol related behaviors in mice selectively bred for high and low activity}

Winona C Booher ${ }^{1,2}$, Aimee L Thomas ${ }^{2}$, Lucy A Hall ${ }^{2}$, Guillermo J Reyes $^{2}$, Marissa A Ehringer ${ }^{1,2}$

${ }^{1}$ Institute for Behavioral Genetics, University of Colorado, Boulder, CO, USA

${ }^{2}$ Department of Integrative Physiology, University of Colorado, Boulder, CO, USA 
Keywords Open-field activity, Anxiety, Alcohol, Mitochondria, Oxidative phosphorylation

Open-field activity (OFA) is widely viewed as a proxy for measuring anxiety-like behavior in mice. DeFries et al. (1978) reported a bidirectional selection experiment that resulted in High and Low Activity (low and high anxiety-like behavior, respectively) strains of mice with 7.8-20 fold differences in OFA. These High and Low Activity strains of mice also exhibit correlated differences in other anxiety-related behaviors including light-dark box, elevated plus maze, mirror chamber test, elevated square maze, and novel object exploration (Henderson et al. 2004). Additionally, due to the high correlation of anxiety and alcohol use disorders (AUDs), we have begun to characterize these strains for several alcohol phenotypes. Using a twobottle choice $(2 \mathrm{BC})$ paradigm, it was revealed that male Low mice consume significantly more alcohol than the male High mice. However, female High mice consume significantly more alcohol than the female Low mice, highlighting a sex difference in $2 \mathrm{BC}$ behaviors. To evaluate acute sensitivity to alcohol, we tested these strains for the loss of righting reflex. Both male and female High mice regain their righting reflex significantly faster than the male and female Low mice, respectively. Additionally, we are testing these strains for acute alcohol induced locomotor sensitization and alcohol metabolism. Finally, because the hippocampus may play a role in both anxiety and AUDs, we have collected alcohol naive brains for hippocampal RNA sequencing, revealing dramatic strain and sex differences in genes associated with mitochondrial function, i.e., up-regulation of several gene ontology terms related to oxidative phosphorylation in female Low mice.

Grant Support: Supported by the University of Colorado internal funds.

\section{Twinning as a phenotype: GWAS and EWAS meta- analyses}

Dorret I. Boomsma ${ }^{1}$, Hamdi Mbarek ${ }^{1,2}$, Jenny van Dongen ${ }^{1}$, Nick G. Martin, the Twinning Genetics Consortium ${ }^{4}$

${ }^{1}$ Netherlands Twin Register, Department of Biological Psychology, Vrije Universiteit Amsterdam, Amsterdam Development and Reproduction Institute, The Netherlands

${ }^{2}$ Qatar Genome Program, DOHA, Qatar

${ }^{3}$ Queensland Institute Medical Research, Brisbane, Australia

${ }^{4}$ http://www.twinningconsortium.org/

Keywords Twinning, Zygosity, Gene-Finding, GWAMA, Epigenetics, EWAS

The classical twin design has been referred to as a perfect 'natural experiment', or the 'working horse' of behavior genetics and continues to be of enormous value to explore total trait heritability, the aetiology of comorbidity and model causality. Twinning as the phenotype of interest in genetic analyses is more rare. We have performed genome-wide association meta-analyses (GWAMA) of spontaneous dizgygotic (DZ) twinning in mothers of twins (MoDZT) and of the proxy phenotype "Are you a DZ twin" and meta-analyzed these results together.

DZ spontaneous twinning is a complex polygenic trait, for which we reported the first 2 replicated genes, FSHB and SMAD3 a few years ago (Mbarek et al. 2016). FSHB had been hypothesised (but never shown) to be implicated in DZ twinning while SMAD3, which regulates the response of the ovaries to $F S H$, was not implicated in twinning before. We have now extended the discovery set for MoDZT and will present the meta-analysis results, combined with the proxy phenotype "being a DZ twin".
In contrast to DZ twinning whose familial / genetic aetiology is well established in family and pedigree studies, the aetiology of monozygotic (MZ) twinning is much more unclear. We also performed genome-wide association studies of "being a MZ twin", obtaining only one genome wide significant finding. However, the first epigenome-wide association study of MZ twinning has generated a plethora of results. We detected a MZ twin-specific DNA methylation signature in whole blood samples, which showed marked replication in 4 independent twin cohorts and in samples from a different cellular lineage.

\section{References}

Mbarek H, Steinberg S, Nyholt DR, Gordon SD, Miller MB, McRae AF, Hottenga JJ, Day FR, Willemsen G, de Geus EJ, Davies GE, Martin HC, Penninx BW, Jansen R, McAloney K, Vink JM, Kaprio J, Plomin R, Spector TD, Magnusson PK, Reversade B, Harris RA, Aagaard K, Kristjansson RP, Olafsson I, Eyjolfsson GI, Sigurdardottir O, Iacono WG, Lambalk CB, Montgomery GW, McGue M, Ong KK, Perry JRB, Martin NG, Stefánsson H, Stefánsson K, Boomsma DI. Identification of Common Genetic Variants Influencing Spontaneous Dizygotic Twinning and Female Fertility. Am J Hum Genet. 2016; 98(5):898-908. doi: 10.1016/j.ajhg.2016.03.008

Grant Support: KNAW Academy Professor Award (PAH/6635) to DIB.

\section{Long-term effects of polygenic propensity for educational attainment on educational attainment mediated by inhibitory control and variation by a family-based intervention}

Giulia A. Borriello ${ }^{1}$, Kit K. Elam ${ }^{2}$, Gianna Rea-Sandin ${ }^{3}$, Kathryn Lemery-Chalfant $^{3}$, Fazil Aliev ${ }^{4,5}$, Danielle Dick ${ }^{5}$, and Thao $\mathrm{Ha}^{3}$ ${ }^{1}$ Department of Psychological and Behavioral Sciences, Indiana University Bloomington, IN, USA

${ }^{2}$ Department of Applied Health Sciences, Indiana University Bloomington, IN, USA

${ }^{3}$ Department of Psychology, Arizona State University, Tempe, AZ, USA

${ }^{4}$ Faculty of Business, Karabuk University, Turkey Department of Psychology, ${ }^{5}$ Virginia Commonwealth University, Richmond, VA, USA

Keywords Educational attainment; Continuous shrinkage; Polygenic Risk Score; Inhibitory control; Adolescence

The majority of studies on educational attainment (EA) focus on direct genetic effects rather than on how etiological influences unfold across development. The current study investigates genetic influences in developmental pathways to EA over a 12-year period in a sample of at-risk youth who participated in a family-based intervention program. We tested for direct effects of a polygenic score (PS) for EA on adulthood EA, mediated by adolescent inhibitory control, and for differences in effects based on intervention status.

Data were from the Project Alliance 1 study (PAL1), a longitudinal randomized trial of 999 adolescents (51\% male) and their families. Participants were randomly assigned to intervention or control conditions of the Family Check-Up (FCU), designed to reduce adolescent problem behavior. Genetic propensity for EA was captured using a continuous shrinkage PS based on a recent GWAS of EA. Adolescent inhibitory control was assessed at age 17 and EA at age 27. All analyses controlled for parents' EA, ethnicity, age, and the first two ancestry principal components. 
The PS directly predicted EA $(B=.15, S E=.04, P<.001)$. In the mediated model the PS predicted inhibitory control $(B=.14, S E=$ $.05, P=002)$, inhibitory control predicted EA $(B=.21, S E=.04, P<$ .001 ; indirect effect $B=.03, S E=.01, P=.02$ ), and a significant direct effect remained $(B=.12, S E=.04, P=.001)$. The association between the PS and EA was significantly different $\left[\chi^{2 \text { diff }}(1)=5.68\right.$, $P=.017]$ in intervention $(B=.04, S E=.06, P=.56)$ and control conditions $(B=.20, S E=.06, P<.001)$. Results highlight individual and FCU mechanisms in pathways to EA.

Grant Support: R01 DA007031, R01 DA036832, Grant DA07031 from the National Institute on Drug Abuse, Grant AA022071 from the National Institute on Alcoholism and Alcohol Abuse.

\section{Turning knowledge of a genetic cause into preventive behavioral interventions: Proactive speech and language therapy for infants with classic galactosemia continues to show signs of effectiveness}

Laurel Bruce ${ }^{1}$, Linda Eng ${ }^{1}$, Sarah Cotter $^{1}$, Hanako Yokoyama ${ }^{1}$, Jacklyn Schur $^{1}$, Inbal Donenfeld-Peled ${ }^{2}$, Beate Peter ${ }^{1,3}$

${ }^{1}$ College of Health Solutions, Arizona State University, Tempe, Arizona, USA

${ }^{2}$ Department of Speech, Language, and Hearing Sciences, Purdue University, West Lafayette, IN

${ }^{3}$ Department of Communication Sciences and Disorders, Saint Louis University, Saint Louis, USA

Keywords Classic galactosemia, Speech sound disorder, Developmental language disorder, Prevention, Babble Boot Camp $\odot$

Speech and language disorders cannot be diagnosed behaviorally until children are old enough to show signs, age 2 to 3 years. In an ongoing clinical trial, we are addressing the question whether these disorders can be mitigated or even prevented if the risk is predictable at birth. Classic galactosemia $(\mathrm{CG})$ is an inborn error of metabolism that is associated with these communication disorders. Infants with $\mathrm{CG}$ have a high (60-80\%) risk for developing these disorders. Because they are identified via newborn screening, this risk is predictable at birth. We created a proactive bundle of routines and activities designed to support typical speech and language development. Infants enter the program before age 5 months and complete the intervention at age 2 years. The Babble Boot Camp (c) is implemented by a speech-language pathologist via parent training. The goals are to shape dyadic interactions, stimulate early vocalizations (coo, babble), support emergence of first words and sentences, and foster vocabulary and syntax growth, all at ages before conventional therapy is available. Here, we report on the progress of 9 children with CG who completed the intervention and one control child with CG. Results show that the treated children produced babble sounds and words with higher articulatory complexity than the control child. Seven children in the treatment group had typical expressive vocabulary sizes whereas the control child and two children in the treatment group had low vocabulary sizes. These results are consistent with beneficial effects of the behavioral intervention on traits of genetic etiology.

Grant Support: 1 R01 HD098253-02.

\section{Current Polygenic Scores Fail to Capture Resilience}

Brianna A. Bucknor ${ }^{1}$, Jaime Derringer ${ }^{1}$
${ }^{1}$ Department of Psychology, University of Illinois at Urbana Champaign, Champaign, Illinois, USA

Keywords Resilience; Psychopathology; Polygenic Scores; Stress; Negative Affect

Stress is a well-known risk factor for the development of psychopathology. However, not everyone who is exposed to stress responds in the same way. Individual differences in resilience to stress reflect dynamic interpersonal and intrapersonal factors. Although resilience has been identified to be moderately heritable, little is known about the genetic variants that may explain this heritability. While there has not yet been a robust genome-wide association study (GWAS) of resilience, existing GWAS of related phenotypes (proxies; e.g., post-traumatic stress disorder), may provide a starting point for developing our understanding of the molecular genetic underpinnings of the observed heritability of resilience. In the HRS dataset, we examined the extent to which 32 polygenic scores (PGS) explained the variance in resilience. PGS were precomputed separately within HRS's pre-defined subsamples of African Americans (N $=1518)$ and European Americans $(\mathrm{N}=7962)$. We then compared the utility of these existing genetic predictors to commonly examined demographic and psychological characteristics, such as socioeconomic status and social support. We found that the psychological correlates consistently and substantially outperformed existing polygenic scores. While psychological variables yielded substantial correlations with resilience, only four of the 32 polygenic scores reached significance but with relatively small effects. Our results indicate that existing polygenic scores are not sufficient to inform our understanding of resilience, nor would they be suitable predictors of individual differences in resilience outcomes. We conclude that a large-scale GWAS of resilience will be necessary to identify the specific genetics underlying the observed heritability of resilience.

Grant Support: HRS is sponsored by the National Institute on Aging (NIA U01AG009740) and is conducted by researchers at the University of Michigan. All data used in this project are publicly available at https://hrs.isr.umich.edu.

\section{Genetic, environmental influences, and brain volume mediation from traumatic stress to PTSD symptoms in children}

Daniel Bustamante ${ }^{1,2}$, Ananda B. Amstadter ${ }^{1,3}$, Michael C. Neale ${ }^{1,3}$

${ }^{1}$ Virginia Institute for Psychiatric and Behavioral Genetics

${ }^{2}$ Integrative Life Sciences Doctoral Program, Virginia Commonwealth University, Richmond, VA, USA

${ }^{3}$ Department of Psychiatry, School of Medicine, Virginia Commonwealth University, Richmond, VA, USA

Keywords Volume, Brain, Mediation, Genetic, PTSD

Volumes of subcortical brain regions of interest (ROIs) have been linked to posttraumatic stress disorder (PTSD). The variation for these phenotypes is heritable; although, most of prior research have assessed adults. Using $\mathrm{ABCD}$ data $\left(\mathrm{N}=11,875, \mathrm{M}_{\mathrm{age}}=9.92\right)$ we extend this work to children to test if subcortical and cortical brain ROIs mediate the relationship between traumatic events (TEs) and PTSD symptoms (PTSDsx), and to determine the genetic and environmental variation for these phenotypes. Volumes of 42 subcortical and 258 cortical ROIs were modeled using machine learning methods to evaluate their role as mediators with an agnostic approach, and to select more parsimonious models. Most of the variance of volumes in subcortical (64.92-89.26\%) and two cortical mediators $(82.80-84.97 \%)$ was explained by additive genetic factors. Whereas, 
environmental factors explained most of the variability in TEs $(\mathrm{C}=$ $.62, \mathrm{E}=.15)$, PTSDsx $(\mathrm{resC}=.19$, resE $=.22)$ and the remainder cortical mediators $(\mathrm{E}=52.98-71.54 \%)$. The direct effects of TEs on PTSDsx were high (.92). However, the indirect effects through the volumes of brain ROIs with the highest mediation effects (e.g., caudate, anterior-cingulate gyrus and sulcus in the left hemisphere, anteromedialtemporal cortex in the right hemisphere) were small to non-influential (subcort: 0.0003-0.0007; cort: 0.0004-0.0011). Genetic and environmental factors influence differently subcortical and cortical volumes, and PTSD phenotypes in children. Longitudinal data will give more information about the effects and direction of causation in this model. The proposed hybrid mediation model is well suited for analyzing the neuroimaging and PTSD phenotypic trajectories, their heritability and environmental contributions with an agnostic approach.

\section{Genetic and environmental predictors of self-worth in young children}

Minhnguyen Cao, MA ${ }^{1}$, Jody M. Ganiban, $\mathrm{PhD}^{1}$, Chang Liu, $\mathrm{PhD}^{1}$, Daniel Shaw, $\mathrm{PhD}^{2}$, David Reiss, $\mathrm{MD}^{3}$, Misaki Natsuaki, $\mathrm{PhD}^{4}$, Leslie Leve, $\mathrm{PhD}^{5}$, Jenae Neiderhiser, $\mathrm{PhD}^{6}$

${ }^{1}$ Department of Psychological \& Brain Sciences, The George Washington University, Washington, DC, USA

${ }^{2}$ Department of Psychology, University of Pittsburgh, Pittsburgh, PA, USA

${ }^{3}$ Child Study Center, School of Medicine, Yale University, New Haven, CT, USA

${ }^{4}$ Department of Psychology, University of California - Riverside, Riverside, CA, USA

${ }^{5}$ Prevention Science Institute, University of Oregon, Eugene, OR, USA

${ }^{6}$ Department of Psychology, Pennsylvania State University, University Park, PA, USA

Keywords Temperament, Self-Worth, Parenting, Surgency, Involvement

Previous research indicates that parental involvement predicts higher self-worth in children, and that self-worth is genetically influenced during childhood. However, few studies consider transactional influences of genetic and environmental (parenting) influences on selfworth. The current study used an adoption design to assess independent and interactive contributions of parental warmth involvement, child surgency, and heritable factors to two domains of early childhood social self-worth: perceived peer acceptance and maternal acceptance. We hypothesized that: (1) child surgency, which captures sociability and positive affectivity, is positively related to children's social self-worth; (2) child surgency mediates associations between heritable tendencies to seek and form positive social relationships (i.e. birth mother agreeableness, behavioral activation) and social self-worth; and (3) adoptive mother involvement and child surgency interact to predict children's social self-worth. Participants included 561 adopted children and their birth and adoptive families from the Early Growth and Development study. When children were 54-months-old, their adoptive mothers reported on their own parenting (maternal involvement); adoptive fathers reported on children's surgency. Children rated their perceived peer and maternal acceptance at age 72 months. Birth mothers reported on their own agreeableness and behavioral activation. Birth mother characteristics predicted children's perceived maternal acceptance, however these effects were not mediated by child surgency. Surgency was positively related to perceived maternal acceptance and peer acceptance, but only when children experienced average to high levels of maternal involvement. This study reaffirmed heritable influences on self-worth, and highlights that child temperament and parenting jointly affect emergent self-worth.

Grant Support: National Institutes of Health, Project ID: 5UH3OD023389-04.

\section{Understanding the effects of neighborhood disadvantage on youth psychopathology}

Sarah L. Carroll ${ }^{1}$, Kelly L. Klump ${ }^{1}$, and S. Alexandra Burt ${ }^{1}$ ${ }^{1}$ Department of Psychology, Michigan State University, East Lansing, Michigan, USA

Keywords Disadvantage, Psychopathology, Genotype-Environment Interplay

In 1942, Shaw and McKay reported that disadvantaged neighborhoods predict youth psychopathology. In the decades since, dozens of papers have confirmed and extended these early results, convincingly demonstrating that disadvantaged neighborhoods predict elevated rates of both internalizing and externalizing disorders, and that they do so across childhood and adolescence. It is not yet clear, however, how neighborhood disadvantage increases psychopathology. The current study sought to fill this gap in the literature by examining the Area Deprivation Index (ADI), a composite measure of census tract disadvantage, as an etiologic moderator of several common forms of psychopathology in two samples of school-aged twins (Total $\mathrm{N}=$ 11,130 twins in 5515 pairs), one of which was enriched for neighborhood disadvantage. Results indicated that, across both samples, additive genetic influences on attention-deficit hyperactivity problems were accentuated in the presence of marked disadvantage, while nonshared environmental contributions to callous-unemotional symptoms increased with increasing disadvantage. However, neighborhood disadvantage had little in the way of moderating effects on the etiology of internalizing symptoms. Such findings point to widespread influences of neighborhood disadvantage on psychiatric and psychological outcomes in childhood, while also suggesting that these effects are phenotype-specific. Implications and future research directions are discussed.

Shaw, C. R., \& McKay, H. D. (1942). Juvenile delinquency and urban areas.

Grant Support: F31HD102094.

\section{Genetic associations between non-cognitive skills and educational outcomes: The role of parental environment}

Perline Demange ${ }^{1,2,3}$, Jouke Jan Hottenga ${ }^{1}$, Abdel Abdellaoui ${ }^{4}$, Margherita Malanchini ${ }^{5,6}$, Ben Domingue ${ }^{7,8}$, Eveline de Zeeuw ${ }^{1,3}$, Kaili Rimfeld ${ }^{5}$, Elsje van Bergen ${ }^{1,3}$, Thalia Eley ${ }^{5,9}$, Robert Plomin', Dorret Boomsma ${ }^{1}$, Gerome Breen ${ }^{5,9}$, Michel Nivard ${ }^{1}$, Rosa Cheesman $^{5}$

${ }^{1}$ Department of Biological Psychology, Vrije Universiteit Amsterdam, The Netherlands

${ }^{2}$ Amsterdam Public Health Research Institute, Amsterdam University Medical Centers, Amsterdam, The Netherlands

${ }^{3}$ Research Institute LEARN!,Vrije Universiteit Amsterdam, The Netherlands

${ }^{4}$ Department of Psychiatry, Amsterdam UMC, University of Amsterdam, Amsterdam, the Netherlands 
${ }^{5}$ Social Genetic \& Developmental Psychiatry Centre, Institute of Psychiatry, Psychology \& Neuroscience, King's College London, UK ${ }^{6}$ Queen Mary University of London

${ }^{7}$ Graduate School of Education, Stanford University

${ }^{8}$ Center for Population Health Sciences, Stanford University

${ }^{9}$ NIHR Maudsley Biomedical Research Centre; South London and Maudsley NHS Trust, UK

Keywords Education, Polygenic scores, Indirect genetic effects, Noncognitive skills, Parenting

Accumulating evidence suggests that parents' genetic variation indirectly influences offspring education via the home environment. No research has assessed contributions of parental genetic variation linked to non-cognitive versus cognitive skills, or compared indirect parental genetic effects across outcome measures, cohorts and analytical methods.

We define the non-cognitive and cognitive heritable contributions to educational attainment using GWAS-by-subtraction, and construct non-cognitive and cognitive skills polygenic scores in British and Dutch cohorts (UK Biobank, TEDS and NTR). We estimate indirect effects of the polygenic scores on educational outcomes using: siblings (all cohorts; $\mathrm{N}=47,459)$, adoptees (UKB; $\mathrm{N}=6407$ ) and parentoffspring trios (NTR; $\mathrm{N}=2534)$. Outcomes include achievement (teacher reports and standardized test scores) at ages 12 and 16, and adult attainment.

Indirect effects of non-cognitive and cognitive skills genetics on offspring education are similar, explaining $\sim 40 \%$ of the total effect of each polygenic score on average. Childhood standardised tests show less influence of parents' non-cognitive genetics than other outcomes (e.g. NTR: $\beta=0.01$ for CITO scores, $\beta=0.13$ for adult attainment). The sibling method gives higher estimates of indirect genetic effects than other approaches, particularly for the non-cognitive polygenic score (e.g. UKB: adoption $\beta=0.02$; sibling $\beta=$ $0.16)$. This likely reflects population stratification and assortative mating.

Heritable non-cognitive and cognitive skills are both involved in how parents create environments that shape offspring education. Estimates of indirect genetic effects are somewhat sensitive to choice of outcome measure, and to patterns of bias affecting particular methods.

Grant Support: RC is supported by an ESRC studentship. PD is supported by the Grant 531003014 from The Netherlands Organisation for Health Research and Development (ZonMW). This research has been conducted using the UK Biobank Resource under Application Number 40310. The Netherlands Twin Registry is supported by NWO Groot (480-15-001/674): Netherlands Twin Registry Repository: researching the interplay between genome and environment and the Avera Institute for Human Genetics, Sioux Falls, South Dakota (USA) for genotyping. We gratefully acknowledge the research program 'Consortium on Individual Development (CID)' which is funded through the Gravitation program of the Dutch Ministry of Education, Culture and Science and the Netherlands Organization for Scientific Research (NWO: 0240-001-003). We gratefully acknowledge 'Open Data Infrastructure for Social Science and Economic Innovations (ODISSEI) (NWO: NRGWI.obrug.2018.008)'. R.P. is supported by a Medical Research Council Professorship award (G19/ 2 ). The authors gratefully acknowledge the ongoing contribution of the participants in the Twins Early Development Study (TEDS) and their families. TEDS is supported by a Programme Grant to R.P. from the UK Medical Research Council (MR/M021475/1 and previously G0901245), with additional support from the US National Institutes of Health (AG046938) and the European Commission (602768; 295366). Data for this study came from the Twins Early Development Study (TEDS). Researchers can apply for access: https://www.teds.ac.uk/researchers/teds-data-access-policy.

\section{Heritable, environmental, and timing effects on the intergenerational transmission of anxiety and depressive symptoms in early adolescence}

Tong Chen ${ }^{1}$, Chang Liu $^{2}$, Leslie D. Leve ${ }^{3}$, Jody M. Ganiban ${ }^{2}$, Misaki N. Natsuaki ${ }^{4}$, David Reiss ${ }^{5}$, Daniel S. Shaw ${ }^{6}$, Jenae M. Neiderhiser ${ }^{1}$ ${ }^{1}$ Department of Psychology, The Pennsylvania State University, University Park, PA, USA

${ }^{2}$ Department of Psychology, George Washington University, Washington, DC, USA

${ }^{3}$ Prevention Science Institute, University of Oregon, Eugene, OR, USA

${ }^{4}$ Department of Psychology, University of California, Riverside, CA, USA

${ }^{5}$ Yale Child Study Center, Yale School of Medicine, New Haven, CT, USA

${ }^{6}$ Department of Psychology, University of Pittsburgh, Pittsburgh, PA, USA

Keywords Anxiety and depressive symptoms; Timing; Sensitive periods; Adoption; Adolescence

Parental anxiety and depressive symptoms are associated with similar problems in children and adolescents; understanding the mechanisms of this transmission could help with prevention of anxiety and depressive symptoms. The present study examined heritable, prenatal and postnatal environmental influences on early adolescent anxiety and depressive symptoms, with a focus on whether different timing of exposure to parent symptoms confers different levels of risk. Participants included 561 families from the Early Growth and Development Study, a prospective parent-offspring adoption design. Heritable risk was indexed by birth mother and father lifetime internalizing problems, prenatal risk was measured by birth mother internalizing problems during pregnancy. Adoptive parent anxiety and depressive symptoms were assessed from child ages 9 months to 11 years and were modeled by the trait-state-occasion model to separate time-invariant and time-varying components. Adolescent anxiety and depressive symptoms were assessed at 11 years; child anxiety and depressive symptoms at age 4.5 were included as controls. Results suggested that maternal anxiety and depressive symptoms at 9 months indirectly influenced adolescent symptoms through early childhood symptoms. Maternal anxiety and depressive symptoms at 11 years was associated with adolescent symptoms concurrently. The timeinvariant component of maternal symptoms had no influence on offspring symptoms. Neither heritable nor prenatal risk was associated with offspring symptoms. Paternal anxiety and depressive symptoms were not associated with offspring symptoms. Results support a sensitive period model for exposure to maternal anxiety and depressive symptoms and provide implications for prevention to focus on within-person changes of maternal symptoms during these sensitive periods.

Grant Support: This project was supported by R01 HD042608, NICHD, NIDA and OBSSR, NIH, U.S. PHS (PI Years 1-5: David Reiss; PI Years 6-10: Leslie Leve), R01 DA020585 NIDA, NIMH and OBSSR, NIH, U.S. PHS (PI: Jenae Neiderhiser), R01 MH092118, NIMH, NIH, U.S. PHS (PIs: Jenae Neiderhiser and Leslie Leve), R01 DK090264, NIDDK, NIH, U.S. PHS (PI: Jody Ganiban), R01 DA035062, NIDA, NIH, U.S. PHS (PI: Leslie Leve), R56 HD042608, NICHD, NIH, U.S. PHS (PI: Leslie Leve), and UG3/UH3 OD023389, Office of the Director, NIH, U.S. PHS (PIs: Leslie Leve, Jenae Neiderhiser and Jody Ganiban). The content is solely the responsibility of the authors and does not necessarily represent the official views of the Eunice Kennedy Shriver National Institute of Child Health \& Human Development, the Office of the Director, or the National Institutes of Health. Environmental influences on Child Health Outcomes (ECHO) 
is a nationwide research program supported by the National Institutes of Health (NIH), Office of the Director to enhance child health.

\section{Differential shared genetic influences of anxiety with problematic alcohol use compared to alcohol consumption}

Sarah Mary Carlton Colbert ${ }^{1,2, *}$, B.A., Scott Funkhouser ${ }^{1}$, Ph.D., Emma C. Johnson ${ }^{3}$, Ph.D., Marissa Ehringer ${ }^{1,4}$, Ph.D., Luke Evans ${ }^{1,2, *}$, Ph.D.

${ }^{1}$ The Institute for Behavioral Genetics, University of Colorado Boulder, Boulder, CO, USA

${ }^{2}$ Department of Ecology and Evolutionary Biology, University of Colorado Boulder, Boulder, CO, USA

${ }^{3}$ Department of Psychiatry, Washington University School of Medicine, St. Louis, MO, USA

${ }^{4}$ Department of Integrative Physiology, University of Colorado Boulder, Boulder, CO, USA

Keywords Anxiety disorders, Alcohol use disorders, Problematic alcohol use, Genetic correlation

Anxiety disorders and alcohol use disorders are common psychiatric disorders. Comorbidity of the two disorders can have tremendous effects on treatment on one or both of the disorders, as well as an individual's social, economic and physical well-being. We estimated genome-wide genetic correlations of anxiety and alcohol use with linkage disequilibrium score regression (LDSC), and found strong and positive correlations with problem use, but not with most consumption measures. Sex-stratified genome-wide genetic correlation analysis found strong positive correlations between males and females for all traits but suggested genetic correlation between alcohol use and anxiety might differ for males and females. Analyses of partitioned heritability using cell type and brain region-specific expression annotations revealed distinct patterns between problematic alcohol use (PAU) and consumption, with consumption traits demonstrating more significant, positive enrichment correlations with anxiety disorders than PAU traits. Partitioning the genetic covariance between traits also identified the amygdala, caudate basal ganglia and frontal cortex as contributing significantly to positive genetic covariance between anxiety and PAU phenotypes. Finally, estimates of local genetic covariance demonstrated divergent genetic covariance profiles of PAU and consumption with anxiety phenotypes, and localized 12 specific regions that likely contribute to both anxiety and alcohol use. This study also serves as a framework for an approach to be used in future analyses of the genetics of comorbid disorders.

\section{Genome-wide association analysis on breastfeeding duration}

Lucía Colodro-Conde ${ }^{1}$, Corinne Carland ${ }^{2,3}$, Sheeva Rajaei ${ }^{2}$, Lavinia Paternoster $^{4}$, Juan F. Sánchez Romera ${ }^{5}$, Juan R. Ordoñana ${ }^{5}$, Michelle Lupton $^{1}$, Themistocles L Assimes ${ }^{2}$, Nicholas G Martin ${ }^{1}$, Sarah E Medland $^{1}$

${ }^{1}$ Department Genetics and Computational Biology, QIMR Berghofer Medical Research Institute, Brisbane, Australia

${ }^{2}$ Department of Medicine, Stanford University School of Medicine, California, USA

${ }^{3}$ Tufts University School of Medicine, Boston, Massachusetts, USA ${ }^{4}$ Medical Research Council Integrative Epidemiology Unit, University of Bristol, Bristol, UK
${ }^{5}$ Department of Psychobiology and Human Anatomy, University of Murcia, Murcia, Spain

Keywords Breastfeeding, GWAS, Women's health

Breastfeeding duration (BFD) is heritable. The first twin study on breastfeeding (Colodro-Conde et al. 2013) showed heritabilities of $49 \%$ for the initiation of breastfeeding in the first child, $44 \%$ for BFD in the first child and 54\% for the average BFD to all children. The heritability was of $53 \%$ for the average BFD in a replication study with an Australian twin sample (Colodro-Conde, et al. 2015). Using an extended twin-family design, Merjonen et al. (2015) showed a heritability estimate of $70 \%$ for the initiation of breastfeeding in Dutch women.

A previous genome-wide scan on BFD found no genetic variants reaching genome-wide significance (Colodro-Conde et al. 2015), although the sample was underpowered $(\mathrm{N}=1521)$. The aim of this project is to identify novel genes that influence breastfeeding duration by carrying out large scale genome-wide association studies (GWAS).

The first analyses focused on the breastfeeding the first-born child and controlled for age at survey time, age at childbirth, twin pregnancy (if known), four first principal components of genetic ancestry, and other covariates relevant to the imputation chips. A GWAS conducted in QIMR samples $(\mathrm{N} \sim 7683)$ did not show any genomewide significant variant associated with the trait. The top ten variants (including the top variant $r s 140939911, p=8.43 \mathrm{E}-08$ ) were located in chromosomes 2,6 , and 12 . These variants did not replicate in the ASLPAC cohort (UK, $\mathrm{N}=3338$ ).

Future work will conduct meta-analyses and will explore genetic correlations with related traits (e.g. breast cancer).

\section{References}

Colodro-Conde, L., et al (2013). Heritability of initiation and duration of breastfeeding behavior. Twin Research and Human Genetics, 16. Colodro-Conde, L., et al (2015). A Twin Study of Breastfeeding With a Preliminary Genome-Wide Association Scan. Twin Research and Human Genetics, 18.

Merjonen, P., et al Does Breastfeeding Behavior Run in Families? Evidence From Twins, Their Sisters and Their Mothers in the Netherlands. Twin Research and Human Genetics, CJO2015

Grant Support: L.C.-C. is supported by a QIMR Berghofer fellowship.

\section{The role of arginine-vasopressin receptor gene polymorphisms in the development of anger-related traits}

Y. Davydova ${ }^{1}$, A. Kazantseva ${ }^{1}$, R. Enikeeva ${ }^{1}$, S. Malykh ${ }^{2}$, M. Lobaskova ${ }^{2}$, E. Khusnutdinova ${ }^{1}$

${ }^{1}$ Institute of Biochemistry and Genetics - Subdivision of the Ufa Federal Research Centre of the Russian Academy of Sciences, Ufa, Russia;

${ }^{2}$ Psychological Institute of the Russian Academy of Education, Moscow, Russia

Keywords Anger, Arginine-vasopressin, Polymorphisms, $\mathrm{G} \times \mathrm{E}$

Anger is an emotional reaction to threat, frustration or social provocation, which modulates an individual's risk for aggression. Molecular-genetic studies demonstrated the involvement of argininevasopressin system in affective psychopathology. However, many of them lack the analysis of $\mathrm{G} \times$ E-interactions thus providing controversial findings. The present study aimed to estimate the main effect of AVPR1A (rs1042615, rs3803107) and AVPR1B (rs28632197, 
$r s 33911258$ ) gene polymorphisms, as well as $\mathrm{G} \times \mathrm{E}$ effects on angerrelated traits in healthy individuals.

The study involved 623 mentally healthy individuals from the Russian Federation ( $81.11 \%$ women; mean age $19.53 \pm 1.75$ years). All participants were of Caucasian origin of several ethnic groups: Russians $(\mathrm{N}=225)$, Udmurts $(\mathrm{N}=218)$ and Tatars $(\mathrm{N}=180)$. Angerrelated traits were assessed using Anger Scale of the Buss Perry Aggression Questionnaire (BPAQ-29). SNPs genotyping was performed used Real-Time PCR. Statistical analysis was conducted with PLINK v.1.9 followed by FDR correction for multiple testing.

Statistical analysis revealed an association of AVPR1A*rs3803107 $T$-allele $\left(\mathrm{P}_{\mathrm{FDR}}=0.03 ; \mathrm{r}^{2}=0.01\right)$ and $A V P R 1 B * r s 33911258 G$-allele $\left(\mathrm{P}_{\mathrm{FDR}}=0.03 ; \mathrm{r}^{2}=0.01\right)$ and decreased anger. Multiple regression analysis demonstrated that smoking $\left(\mathrm{P}_{\mathrm{FDR}}=0.01 ; \mathrm{r}^{2}=0.16\right)$, birth season $\left(\mathrm{P}_{\mathrm{FDR}}=0.04 ; \mathrm{r}^{2}=0.04\right)$ and place of residence $\left(\mathrm{P}_{\mathrm{FDR}}=0.04 ; \mathrm{r}^{2}\right.$ $=0.02)$ significantly modulated association of $A V P R 1 B * r s 33911258$ and anger, while birth season $\left(\mathrm{P}_{\mathrm{FDR}}=0.03 ; \mathrm{r}^{2}=0.05\right)$ significantly modulated association on the AVPR1A*rs3803107 and anger in total sample.

Our findings provide evidence for specific effect of several environmental factors (smoking, birth season and place of residence) on the associations AVPRIA and AVPRIB gene polymorphisms and anger-related traits.

Grant Support: This study was supported by the grant of the Bashkortostan Republic to young scientists in 2020 (State Contract No. 9GR) and by the grant of the Russian Foundation for Basic Research (Project No. 17-29-02195 ofi_m).

\section{Systematic review: How the ADHD polygenic risk scores add to our understanding of ADHD and other traits}

Nora de Bode, Angelica Ronald, Tinca JC Polderman

Department of Psychological Sciences, Birkbeck University of London, London, United Kingdom

Complex Trait Genetics, Center for Neuroscience and Cognitive Research, Vrije Universiteit Amsterdam, Amsterdam, The Netherlands

Department of Child and Adolescent Psychiatry, Amsterdam UMC, Amsterdam, The Netherlands

Keywords ADHD, Polygenic score, Review, Prediction, Psychiatry

The latest genome-wide association study (GWAS) offers the clearest picture yet of the common genetic architecture underlying ADHD. As such, a fast-growing literature on how ADHD polygenic risk impacts child development is emerging. Our systematic review provides a comprehensive overview of studies using the ADHD polygenic risk score (PRS) to predict ADHD and other traits. Pubmed, Embase and PsychoInfo were systematically searched. A quality assessment of all studies was conducted. Studies were excluded when a) the predictor was not the latest ADHD PRS; b) the PRS was not based on genomewide results; or c) the study was a review. Initially, 138 studies were retrieved [dd. Feb 22nd, 2020] in our initial search resulting in 35 eligible studies; a second search [dd June 6th 2020) retrieved a further 8 studies, of which four were eligible. Thus, we identified 39 studies that met our inclusion criteria. The quality assessment, conducted by two of the authors, revealed good to excellent quality for the majority of studies. There was a diverse array of outcome measures including clinical ADHD status, ADHD symptoms, cooccurring psychopathology and bullying, addictive behaviors, executive functioning and intelligence, brain measurements, DNA methylation and study participation, of which the majority showed significant association with the ADHD PRS. In conclusion, a wealth of new literature on the ADHD PRS has recently emerged. Our review demonstrates that many studies of high quality show that polygenic risk underlying ADHD predicts a range of phenotypes.

\section{Genetic evidence for the overlap and bidirectional effects between resilience and well-being.}

L.P. de Vries ${ }^{1,2}$, B.M.L. Baselmans ${ }^{3}$, J.J.Luykx ${ }^{4}$, E. de Zeeuw ${ }^{1}$, C. Minică $^{1}$, E.J.C. de Geus ${ }^{1}$, C.H. Vinkers ${ }^{5,6}$, \& M. Bartels ${ }^{1,2}$

${ }^{1}$ Department of Biological Psychology, Vrije Universiteit Amsterdam, The Netherlands

${ }^{2}$ Amsterdam Public Health Research Institute, Amsterdam University Medical Centres, Amsterdam, The Netherlands

${ }^{3}$ Institute for Molecular Bioscience, The University of Queensland, Brisbane, QLD, Australia

${ }^{4}$ Department of Psychiatry, UMC Utrecht, Department of Translational Neuroscience, UMC Utrecht, The Netherlands

${ }^{5}$ Department of Psychiatry, Amsterdam UMC, Location VUmc, The Netherlands

${ }^{6}$ Department of Anatomy and Neurosciences, Amsterdam UMC, Location VUmc, The Netherlands

Keywords Resilience, Well-being, Twin-sibling model, polygenic scores, MR-DoC model

Resilience and well-being are strongly related, happier people are known to show more resilience after stressful life events or trauma and vice versa. Less is known about the underlying sources of overlap and causality between the constructs. In a sample of 11.304 twins and 2.572 siblings from the Netherlands Twin Register, we investigated the overlap and possible direction of causation between resilience (operationalized as: not developing psychiatric symptoms despite negative life events) and well-being in multiple ways using longitudinal data, twin-sibling models, polygenic risk scores, and the Mendelian Randomization Direction of Causality (MR-DoC) model. We defined resilience as the difference between the actual level of anxious-depressed symptoms and the predicted level based on the number of life events experienced. Well-being was measured using the Satisfaction with Life Scale. The family-based genetic modelling showed strong phenotypic ( .50), genetic (.71), and environmental (.93) correlations between resilience and well-being and a large genetic $(51 \%)$ contribution to the covariance. The causality and MRDoC results are in line with a bidirectional causal relation. In summary, there is a large overlap between well-being and resilience, but the traits also appear to be different constructs that influence each other bidirectionally. As resilience and well-being are both negatively related to psychopathology, the high overlap and bidirectionality can have important implications for interventions to prevent or lower vulnerability for psychopathology.

Grant Support: This work is supported by an ERC Consolidation Grant (WELL-BEING 771057, M. Bartels), NWO Large Investment Grant (NTR: 480-15-001/674)) and ZonMW Addiction Program (31160008)

\section{Parental social class, genes and luck: a cohort analysis of equality of opportunity in education}

\author{
Rita Dias Pereira ${ }^{1,2}$ \\ ${ }^{1}$ Erasmus School of Economics, Erasmus University Rotterdam \\ ${ }^{2}$ Tinbergen Institute
}


Keywords Equality of Opportunity, Education, Parental Social Cass, Genes, Luck

This paper evaluates fairness in education across cohorts in the U.S through the lens of the equality of opportunity framework. It is the first study to add an explicit measure of genetic circumstances- the educational attainment polygenic score (EA PGS) - to the literature of equality of opportunity. Two ethical viewpoints are considered: one where genetic endowments a standard circumstance. The second one where genetic endowments are also perceived as circumstance, but one for which we do not wish to equalize or de-correlate educational outcomes. Testable empirical conditions of both viewpoints are derived. Results suggest a clear increase of equality of opportunity for younger cohorts (1948-1953) as compared to older cohorts (1926-1930) with respect to secondary education, irrespective of the ethical framework considered. Results with respect to higher education are nuanced; if the EA PGS is defined as a circumstance, the equality of opportunity has decreased, with the total share of explained variance by circumstances increasing from $19.7 \%$ to $24.1 \%$. However, the conclusions differ if one considers that differences in educational outcomes due to differences in the EA PGS are fair: the explained variance of circumstances falls from $14,2 \%$ to $8,1 \%$. Further, simply relying on the explained variance of childhood SES alone might be misleading. In particular, the seeming increase in the explained variance of childhood SES is explained by the increasing correlation between the two circumstances; younger cohorts with higher childhood SES are also more likely to have a higher EA PGS.

Grant Support: Norface-Dial (Research project: Gene-Environment interplay in the generation of health and education inequalities).

\section{Genomic analysis of $\sim 1.5$ million people uncovers genes associated with externalizing}

Richard Karlsson Linnér ${ }^{1, \dagger, *}$, Travis T. Mallard ${ }^{2, \dagger}$, Peter B. Barr ${ }^{3, \wedge}$, Sandra Sanchez-Roige ${ }^{4, \wedge}$, Irwin Waldman ${ }^{5,+}$, Abraham A. Palmer ${ }^{4, *}$, K. Paige Harden ${ }^{2, *}$, Philipp D. Koellinger ${ }^{1,+}$, \& Danielle M. Dick ${ }^{3, *}$ ${ }^{1}$ Department of Economics, Vrije University Amsterdam, Amsterdam, Netherlands

${ }^{2}$ Department of Psychology, The University of Texas at Austin, Austin, Texas, USA

${ }^{3}$ Department of Psychology, Virginia Commonwealth University, Richmond, Virginia, USA

${ }^{4}$ Department of Psychiatry, University of California San Diego, La Jolla, California, USA

${ }^{5}$ Department of Psychology, Emory University, Atlanta, Georgia, USA

†Joint first authors.

Joint second authors.

*Joint senior authors.

${ }^{+}$Substantive contributions to design of the project.

Keywords Externalizing, Impulsivity, GWAS, Substance use disorders, Childhood behavior disorders

Externalizing refers to behaviors and disorders related to self-regulation, such as substance use disorders, childhood behavior problems, and risky sexual behavior, which have profound individual and societal costs. Previous twin-family studies have indicated that there is a strong heritable component shared across behaviors and disorders characterized by behavioral undercontrol. In this project, we apply multivariate genomic structural equation modeling to study the underlying genetic liability that influences a spectrum of externalizing disorders and behaviors. Our results identify 579 independent loci associated with externalizing liability. The identified genes are largely expressed in the brain and involved in neurodevelopment. The polygenic risk score explained $10 \%$ of the variance in externalizing in independent samples and was also significantly associated with a wide range of behaviors and disorders on the externalizing spectrum, including multiple substance use and psychiatric disorders, childhood and adult antisocial behavior, risky sexual behaviors, and suicide attempts; economic and social correlates of externalizing, including criminal justice system involvement and employment history; and a wide range of biomedical outcomes related to behavioral undercontrol, including HIV infection, diabetes and obesity, cirrhosis of liver, and lung cancer. These analyses demonstrate the wide-reaching effects of a genetic liability for externalizing.

Grant Support: The Externalizing Consortium has been funded in part by the National Institute of Alcohol Abuse and Alcoholism through R01AA015146, P50AA0022527, and K02AA018755 to DMD, and a European Research Council Consolidator Grant (647648 EdGe) to PK.

\section{Preschool pretend play and memory development: twins versus singletons}

Lisabeth F. DiLalla ${ }^{1}$, Matthew R. Jamnik ${ }^{2}$

${ }^{1}$ Department of Family and Community Medicine, Southern Illinois University School of Medicine, Carbondale, Illinois, USA

${ }^{2}$ Department of Psychology, Southern Illinois University, Carbondale, Illinois USA

Keywords Pretend play; Preschoolers; Memory; Household chaos; Twins

Household chaos and family structure are related to cognitive outcomes in preschoolers, but less is known about how they affect play behaviors. We hypothesized that: (1) twins are more cognitively at risk than singletons because of increased likelihood of birth complications, chaotic home environment, and shared (therefore decreased) parental attention; (2) twins have more advanced play skills because of their 'built-in' peer; (3) play skills will moderate the relationship between family chaos and children's cognitive skills.

Twins and singletons from the Southern Illinois Twins/Triplets and Siblings Study (DiLalla and Jamnik 2020) were tested at 3 years on a pretense play task and at ages 3 and 4 years on the Stanford-Binet Memory for Sentences subtest. Parents of twins (but not singletons) completed a measure of home chaos at age 3 .

Twins scored significantly higher than singletons on play and significantly lower on memory. Additionally, play and memory were both significantly heritable; however, this did not appear to be the result of shared genes. Multilevel modeling showed a significant interaction between home chaos and play scores predicting change in memory scores; as household chaos increased, twins with poorer pretend play skills showed significantly less improvement in memory over time.

Thus, twins may overcome their 'risks' via having a 'built-in' peer for play skills; however, if their play skills are poorer, those in chaotic homes are at greatest cognitive risk.

\section{References}

DiLalla, L.F. \& Jamnik, M.R. (2020). The Southern Illinois Twins/ Triplets and Siblings Study (SITSS): A longitudinal study of early child development. Twin Research and Human Genetics.

Grant Support: Funding for this study came from an SIU Special Research Project Grant and a grant from the Central Research Committee of the SIU School of Medicine to LFD. 


\section{Linking cancer and twin registry data to evaluate cancer risk in a United States sample}

Elizabeth K Do ${ }^{1,2}$, Hermine H Maes ${ }^{2,3}$, John M Quillin ${ }^{2,3}$, Kandace P McGuire $^{2}$, David C Wheeler ${ }^{2,4}$, Bassam Dahman ${ }^{1,2}$, Anne Morris ${ }^{5}$, Emily C Lilley ${ }^{5}$, Renolda Gelzinis ${ }^{5}$, and Bernard F Fuemmeler ${ }^{1,2}$ ${ }^{1}$ Department of Health Behavior \& Policy, Virginia Commonwealth University, Richmond, Virginia, USA; ${ }^{2}$ VCU Massey Cancer Center, Virginia Commonwealth University, Richmond, Virginia, USA; ${ }^{3}$ Department of Human and Molecular Genetics, Virginia Commonwealth University, Richmond, Virginia, USA; ${ }^{4}$ Department of Biostatistics, Virginia Commonwealth University, Richmond, Virginia, USA; ${ }^{5}$ Mid-Atlantic Twin Registry, Virginia Commonwealth University, Richmond, Virginia, USA

\section{Keywords Twin registry, Cancer registry, Cancer risk}

Familial clustering has been observed for a number of cancers, such as that of the breast, colon, prostate, and lung (Ahlbom et al. 1997). However, it can be difficult to distinguish between genetic and environmental factors contributing to risk for cancer within family studies, due to the aggregation of both environmental and cultural influences within families. Prior research has focused on individual data obtained from Nordic countries (Mucci et al. 2016), which might not be generalizable to other populations. To address this limitation, we looked towards linking historical twin registry survey data and cancer registry data spanning from 1970 to 2019. Virginia Commonwealth University maintains the Mid-Atlantic Twin Registry (MATR), one of the largest twin registries in the United States. We were able to link data across 16,343 individual twins with confirmed zygosity (excluding higher order multiples) and cancer status. From those for whom we had cancer status, 2627 had ever been diagnosed with cancer in their lifetime. The most prevalent cancer types were: prostate, breast, lung, colon or rectum, and gynecological (including cervix, corpus uteri, and ovary). Univariate twin analyses were conducted to estimate causes of variation in cancer status and co-twin control analyses were done to evaluate the association of environmental risk factors with different types of cancer. Results suggest that genetic and shared environmental factors influence cancer risk and that that environmental factors play a larger role in breast, lung, and colon and rectal cancers. Results also demonstrate the unique opportunity provided by linking twin and cancer registry data to describe factors that drive cancer risk among the population and advance cancer control science and research.

\section{References}

Ahlbom, A. et al. Cancer in Twins: Genetic and Nongenetic Familial Risk Factors. JNCI J. Natl. Cancer Inst. 89, 287-293 (1997).

Mucci, L. A. et al. Familial Risk and Heritability of Cancer Among Twins in Nordic Countries. JAMA 315, 68-76 (2016).

Grant Support: This research is supported by Virginia Commonwealth University Massey Cancer Center, under the MCC Pilot Program.

\section{General factor model of substance use co-morbidity}

Deepika R Dokuru ${ }^{1}$, Maia Frieser ${ }^{1}$, John $\mathrm{K}$ Hewitt ${ }^{1}$, Michael C Stallings ${ }^{1}$

${ }^{1}$ Department of Psychology and Neuroscience, and Institute for Behavioral Genetics, University of Colorado Boulder, USA

Keywords Substance use, Comorbidity, Genetic etiology, Twins
While the high rate of comorbidity among substance use disorders is well described, its detailed etiology is mostly unknown. Prior work by Vrieze et al. (2012) explored the longitudinal influence of environmental and genetic factors on a common factor underlying dependence symptom counts for tobacco, alcohol, and cannabis in twins. They found that a general factor had the greatest influence on substance dependence symptoms in adolescence but decreased over time with substance-specific influences increasing in adulthood. We set forth to replicate and expand their analysis by incorporating other substances along with alcohol, tobacco, and cannabis. We used data from the Colorado longitudinal twin studies $(\mathrm{N}=2884)$. The samples were assessed at three waves with mean ages of 14.99, 20.05, and 25.51 respectively. Analyses utilized transformed (ordinal) DSM dependence counts for tobacco, alcohol and cannabis. Dependence symptom counts for other substances were pooled and the maximum dependence count was used. Consistent with Vrieze et al. (2012), a phenotypic confirmatory factor analysis (CFA) indicated that the common latent factor shows the highest common loadings for all substances at wave 1 (adolescence). Common factor loadings decrease and specific loadings increase overtime. Analyses are being extended to explore sex-limitation and common pathway ACE models to investigate the genetic and environmental factor structure over time.

\section{References}

Vrieze, S. I., Hicks, B. M., Iacono, W. G., \& McGue, M. (2012). Decline in genetic influence on the co-occurrence of alcohol, marijuana, and nicotine dependence symptoms from age 14 to 29 . The American Journal of Psychiatry, 169(10), 1073-1081. https://doi.org/10.1176/appi.ajp.2012.11081268

Grant Support: P50 DA011015, T32DA017637.

\section{Heteroscedastic regression modeling elucidates gene- by-environment interaction}

\author{
Benjamin W Domingue ${ }^{1}$, Klint Kanopka ${ }^{1}$, Sam Trejo ${ }^{1}$, Elliot M \\ Tucker-Drob ${ }^{2}$ \\ ${ }^{1}$ Graduate School of Education, Stanford University, Stanford CA \\ ${ }^{2}$ Department of Psychology, University of Texas at Austin, Austin \\ TX
}

Keywords Gene-by-environment, $\mathrm{G} \times \mathrm{E}$, Polygenic Score, BMI

Gene-by-environment interaction $(\mathrm{G} \times \mathrm{E})$ is inferred when a genetic measure accounts for differing amounts of variance in a phenotype across levels of the environment. However, total variance in the phenotype may shift as a function of the environment irrespective of its genetic etiology such that the proportional effect of the genetic measure is constant. We expand the traditional $\mathrm{G} \times \mathrm{E}$ regression model to directly model heteroscedasticity, and we derive a test statistic, $\xi$, for inferring whether $\mathrm{G} \times \mathrm{E}$ can be plausibly attributed to a more general effect of the environment on the dispersion of the phenotype. In simulation studies, we demonstrate that the test statistic can be utilized, along with evaluations of other model parameters, to adjudicate between multiple hypotheses regarding $\mathrm{G} \times \mathrm{E}$. We then apply this method to test whether previous reports of increasing penetrance of polygenic scores for BMI in more recent birth cohorts may be due to more general secular increases in the variance of BMI. We provide an $R$ function for estimating heteroscedastic $G \times E$ regression models and calculating $\xi$.

Grant Support: This work has been supported in part by the National Science Foundation Graduate Research Fellowship Program under Grant No. DGE-1656518 (ST), by the Institute of Education Sciences 
under Grant No. R305B140009 (ST), NIH grants R01AG054628 and R01HD083613 (EMTD), and by the Jacobs Foundation (EMTD). Any opinions expressed are those of the authors alone and should not be construed as representing the opinions of either foundation.

\section{The impact of receiving genotypic information for psychiatric conditions: a literature review}

\author{
M. Driver ${ }^{1}$, S. Kuo ${ }^{2}$, D. Dick ${ }^{1,2}$ \\ ${ }^{1}$ Virginia Commonwealth University School of Medicine, Depart- \\ ment of Human and Molecular Genetics, Richmond, Virginia, 23298, \\ USA \\ ${ }^{2}$ Virginia Commonwealth University, Department of Psychology, \\ Richmond, Virginia, 23284, USA
}

Keywords Psychiatric genetics, Genetic testing, Personalized feedback, Genetic literacy

Genome-wide association studies are rapidly advancing our understanding of the genetic architecture of complex disorders, including many psychiatric conditions such as major depression, schizophrenia, and substance use disorders. One common goal of genome-wide association studies is to use findings for enhanced clinical prediction in the future, which can aid in identifying at-risk individuals to enable more effective prevention screening and treatment strategies. In order to achieve this goal, we first need to gain a better understanding of the issues surrounding the return of complex genetic feedback for psychiatric conditions. Here, we review and summarize the current literature on the impact of receiving genotypic information for psychiatric conditions on affect, thoughts, and behavior. Reviewed literature shows that genotypic information indicating increased risk for a psychiatric condition lowers an individual's confidence to control behavior, reduces self-agency, and negatively impacts affect and retrospective recall of mood. Individuals may believe that a change in behavior is important; however, the current literature does not provide evidence that genotypic information indicating increased risk for a psychiatric condition is strongly associated with behavior change. Some evidence suggests that education about non-deterministic nature of genetic influences can mitigate negative effects on selfagency. Due to limitations of these studies, future research will need to investigate the impact of receiving true complex genotypic information for psychiatric conditions on a person's behavior and psychological state over an extended period of time and identify effective educational materials that will mitigate potential negative outcomes.

Grant Support: NIH Grant K02AA018755 (PI: Dick) and NIH Grant R34AA027347 (PI: Dick/Langberg).

\section{Associations between ADHD and medical disorders in adulthood: a large-scale genetically informed Swedish register study}

Ebba Du Rietz ${ }^{1}$, Isabell Brikell ${ }^{2}$, Agnieszka Butwicka ${ }^{1}$, Ralf KujaHalkola $^{1}$, Henrik Larsson ${ }^{1,3}$

${ }^{1}$ Department of Medical Epidemiology and Biostatistics, Karolinska Institutet, Stockholm, Sweden

${ }^{2}$ The National Centre for Register-based Research, Department of Economics and Business Economics, Business and Social Science. Aarhus University, Aarhus, Denmark

${ }^{3}$ School of Medical Sciences, Örebro University, Örebro, Sweden
Keywords ADHD, Comorbidity, Somatic, Genetics, Sibling Design

Only a limited number of medical disorders have been thoroughly studied in relation to ADHD, and knowledge is especially lacking for disorders that develop in older ages. This study aimed to map out the phenotypic and aetiologic associations between ADHD and a wide range of medical disorders across adulthood.

Full- and maternal half-siblings ( $\mathrm{N}=4,288,451$ pairs), aged 18-81 years, were identified from Swedish Population Registers and linked to ICD-diagnoses from National Patient Registers. Logistic regression was used to estimate associations between ADHD and 35 medical disorders (8 disease groups) within-individuals, and across full- and half-siblings. Quantitative genetic modelling was performed to estimate genetic and environmental contributions to the associations with ADHD.

Adults with ADHD had increased risk for most medical disorders (34/35), showing the strongest associations with nervous system (OR $=3.27)$ and respiratory $(\mathrm{OR}=2.49)$ disease groups. Significantly $(P<$ 0.001 ) stronger associations were found between full-siblings than half-siblings for nervous system, respiratory, musculoskeletal and metabolic disease groups. Subsequent quantitative genetic modelling showed that these associations with ADHD were largely explained by shared genetic factors, with the exception for nervous system disorders.

Individuals with ADHD are at increased risk for a range of medical disorders, with long-term aspects into adult life. While numerous associations between ADHD and medical disorders were largely driven by genetic factors, others, such as nervous system and ageing disorders were mainly driven by individual-specific environmental factors. This mapping of aetiological sources of covariance can guide future research aiming to identify specific mechanisms that contribute to risk for medical disorders in ADHD.

Grant Support: Ebba Du Rietz is supported by Grant 2019-01172 from the Swedish Research Council for Health, Working Life, and Welfare, and Grant 2019-00482 from Fredrik och Ingrid Thurings Stiftelse. Henrik Larsson acknowledges financial support from the Swedish Research Council (2018-02599) and the Swedish Brain Foundation (FO2018-0273).

\section{The effect of smoking during pregnancy on severity and directionality of externalizing and internalizing symptoms: a genetically-informed approach}

Mikael O Ekblad ${ }^{1,2}$, Kristine Marceau ${ }^{1}$, Emily Rolan ${ }^{1}$, Rohan HC Palmer $^{3}$, Alexandre Todorov ${ }^{4}$, Andrew C Heath ${ }^{4}$, Valerie S Knopik ${ }^{1}$ ${ }^{1}$ Department of Human Development and Family Studies, Purdue University, West Lafayette, Indiana, USA

${ }^{2}$ Department of General Practice, Turku University and Turku University Hospital, Turku, Finland

${ }^{3}$ Behavioral Genetics of Addiction Laboratory, Department of Psychology, Emory University

${ }^{4}$ Department of Psychiatry, Midwest Alcoholism Research Center, Washington University School of Medicine, St Louis

Keywords Genetically-Informed Designs, Prenatal Exposure, Tobacco, Health Consequences, Sibling Comparison

Objective To examine the association between maternal smoking during pregnancy (SDP) and (i) severity and (ii) directionality of externalizing and internalizing symptoms in a sample of sibling pairs while rigorously controlling for familial confounds.

The Missouri Mothers and Their Children Study includes families (N $=173$ ) selected for sibling pairs (aged 7 to 16 years) discordant for SDP. This sibling comparison study is designed to disentangle the 
effects of SDP from familial confounds. An SDP severity score was created for each child using a combination of SDP indicators (timing, duration, and amount). Principal component analysis of externalizing and internalizing behavior, assessed with the Child Behavior Checklist and Teacher Report Form, was used to create symptom severity and directionality scores.

The variance in severity and directionality scores was primarily a function of differences between siblings ( $71 \%$ and $85 \%$, respectively) rather than differences across families ( $29 \%$ and $15 \%$, respectively). The severity score that combines externalizing and internalizing symptom severity was not associated with SDP. However, a significant within-family effect of SDP on symptom directionality $(b=0.07$, $\mathrm{p}=0.04)$ was observed in the sibling comparison model.

The positive directionality score indicates that SDP is associated with differentiation of symptoms towards externalizing rather than internalizing symptoms after controlling for familial confounds with a sibling comparison model. This supports a potentially causal relationship between SDP and externalizing behavior.

Grant Support: Funded by the Foundation for Pediatric Research (Finland, M.O.E.), the Orion Research Foundation sr (M.O.E.), the Emil Aaltonen Foundation (M.O.E.), the Paulo Foundation (M.O.E.), the Maud Kuistila Memorial Foundation (M.O.E.), and the Turku University Hospital Research Foundation (M.O.E.), and the National Institute of Health; Grant numbers: DA023134 (V.S.K.), DA17671 (V.S.K.), AA07728 (A.C.H.), AA09022 (A.C.H.), AA11998 (A.C.H.), HD049024 (A.C.H.), AA017688 (A.C.H.), AA021492 (A.C.H.), R01DA04272 (R.H.), K01DA039288 (K.M.). None of the funding sources had a role in study design, data collection, analyses, interpretation of data, writing of the report, or decision to submit this manuscript for publication.

\section{Do modifiable risk factors moderate the effect of APOE on cognitive decline?}

Jarrod M Ellingson ${ }^{1,2}$, Richard C Border ${ }^{2}$, Jeff M Lessem² ${ }^{2}$ David J Llewellyn $^{3}$, Elzbieta Kuzma ${ }^{3}$, Matthew C Keller ${ }^{2}$

${ }^{1}$ Department of Psychiatry, University of Colorado School of Medicine, Aurora, Colorado, USA;

${ }^{2}$ Institute for Behavioral Genetics, University of Colorado, Boulder, Colorado, USA

${ }^{3}$ Institute of Health Research, University of Exeter Medical School, Exeter, Devon, UK

\section{Keywords $\mathrm{G} \times \mathrm{E}$, ApoE, Dementia, UK Biobank, Education}

There is an extensive literature examining gene-environment interactions $(\mathrm{G} \times \mathrm{E})$ on psychopathology, such as risk from individual genes being moderated by modifiable risk factors. The general pattern emerging from this literature is that $\mathrm{G} \times \mathrm{E}$ effects fail to replicate across samples/research groups. One possibility for failed $\mathrm{G} \times \mathrm{E}$ replications may be that a vast majority of genes associated with psychopathology confer very small risk, and prior studies have been underpowered for $\mathrm{G} \times \mathrm{E}$ investigations. Importantly, the ApoE gene, linked to dementia, stands in stark contrast to other psychopathologylinked genes. Specifically, heterozygotes for the ApoE risk polymorphism $(\varepsilon 4)$ have a three-fold increase in risk and homozygotes have a 15 -fold increase in risk for developing late-onset dementia (vs. ع3/ع3 - those with no risk polymorphisms). Thus, the ApoE gene presents a unique opportunity to examine $\mathrm{G} \times \mathrm{E}$ effects with modifiable factors (e.g., diet, exercise), thereby informing patient decision making regarding their lifestyle. We applied survival analyses to the UK Biobank $(\mathrm{N}=417,323 ; M$ age $=66)$ to examine ApoE-by-modifiable risk effects on dementia. First, main effect suggested that modifiable factors can increase dementia risk, including: current depression, history of depression/anxiety, diabetes, pack years of cigarette use, and body mass index. Further, some modifiable factors may decrease dementia risk, including: grip strength, vitamin D levels (via blood test), education, social support, and hormone replacement therapy (in women only). Finally, after correcting for multiple testing, interaction models suggested that the risk conferred by the ApoE gene varied as a function of participant education. Specifically, ApoE risk was attenuated as participant educational attainment increased.

Grant Support: K23AA026635 (JME).

\section{The role of inflammatory system genes in non-verbal intelligence in mentally healthy individuals}

Renata Enikeeva ${ }^{1}$, Anastasiya Kazantseva $^{1}$, Yuliya Davydova ${ }^{1}$, Zalina Takhirova ${ }^{2}$, Rustam Mustafin ${ }^{3}$, Marina Lobaskova ${ }^{4}$, Sergey Malykh $^{4}$, Elza Khusnutdinova ${ }^{1}$

${ }^{1}$ Institute of Biochemistry and Genetics - Subdivision of the Ufa Federal Research Centre of the Russian Academy of Sciences, Ufa, Russian Federation;

${ }^{2}$ Russian Academy of Education, Moscow, Russian Federation; ${ }^{3}$ Department of Medical Genetics and Fundamental Medicine, Bashkir State Medical University, Russian Federation;

${ }^{4}$ Psychological Institute of the Russian Academy of Education, Moscow, Russian Federation.

Keywords Cognitive ability, Oxytocin, Gene x Environment interactions, Birth order, Haplotype

A multifactorial nature of cognitive abilities (including non-verbal intelligence) suggests the involvement of multiple genes of small effect and environmental factors in its development, thus requiring simultaneous examination of genetic and environmental factors. Recently, pathways involved in immune function and inflammatory response, especially interleukin and cytokine signaling (i.e. TNF, $I L 1 B, C R P)$ were related to cognitive impairments. However, the data on their involvement in cognitive abilities is scarce.

In order to assess the possible involvement of inflammatory genes in cognitive processing, the present study aimed to estimate both the main effect of $I L 1 B, C R P$ and $T N F$ gene polymorphisms and $\mathrm{G} \times \mathrm{E}$ interactions in individual differences in non-verbal intelligence in healthy individuals.

The study included 897 mentally healthy individuals (79\% women; $19.74 \pm 1.51$ years) of Caucasian origin (428 Russians, 200 Tatars, 117 Udmurts, and 152 of mixed ethnicity) from Russia. The assessment of non-verbal intelligence was conducted via Raven's progressive matrices. SNPs genotyping was performed using PCRbased KASP genotyping technology on "CFX96" DNA Analyzer (BioRad, USA). Statistical analysis included multiple linear/logistic regression (FDR-correction, PLINK v.1.09). Genotypes and 21 environmental parameters served as independent factors and nonverbal intelligence as dependent variable.

Statistical analysis revealed association of increased non-verbal intelligence and TNF rs1041981 A-allele in men $\left(\beta=1.75 ; \mathrm{P}_{\mathrm{FDR}}=\right.$ $0.035)$ and $I L 1 B$ rs 16944 A-allele in Tatars $\left(\beta=1.645 ; \mathrm{P}_{\mathrm{FDR}}=0.005\right)$. Moreover, $\mathrm{G} \times \mathrm{E}$ model demonstrated that sibship size modulated the association of $T N F$ rs 1041981 A-allele and non-verbal intelligence ( $\beta$ $=1.90 ; \mathrm{P}=0.005$ ).

The present study indicated the involvement of inflammatory system genes $(T N F, I L 1 B)$ in individual variation in cognitive abilities in healthy individuals.

Grant Support: The present study was supported by the Russian Science Foundation (Project No. 17-78-30028). 


\section{Longitudinal dynamic relationship between temperament and cognition in childhood: the Louisville Twin Study}

\author{
Deborah Finkel ${ }^{1,2}$, Deborah W. Davis ${ }^{3}$, Eric Turkheimer ${ }^{4}$, Chris R. \\ Beam $^{5}$ \\ ${ }^{1}$ Department of Psychology, Indiana University Southeast, New \\ Albany, Indiana, USA \\ ${ }^{2}$ Institute for Gerontology, Jönköping University, Jönköping, Sweden \\ ${ }^{3}$ University of Louisville, Louisville, Kentucky, USA \\ ${ }^{4}$ University of Virginia, Charlottesville, Virginia, USA \\ ${ }^{5}$ University of Southern California, Los Angeles, California, USA
}

Keywords Cognition, Temperament, Childhood, Longitudinal, Bidirectional

Recognition that "non-cognitive" skills may impact cognitive outcomes has increased in recent decades. Research on the role of temperament in cognitive development typically focuses on the roles that reactivity, persistence, and approach play in school readiness and learning abilities. Few studies account for genetic associations or the possibility of reverse causation. Application of the bivariate dual change score model allows for investigation of genetic and environmental contributions to relationships between trajectories of change in temperament and cognition in childhood. Longitudinal assessments of mental development (MD) and temperament were collected from 1409 twins from ages 3 to 10 years as part of the Louisville Twin Study ( $70 \%$ with 3 or more waves). Factor analysis of 8 temperament scales from the McDevitt Style Questionnaire and the Temperament in Middle Childhood Questionnaire resulted in 3 factors consistent across waves: reactivity (intensity, distress, and threshold), persistence (persistence and activity), and approach (approach, adaptability, and mood). Mental development was assessed with the age-appropriate scale (Stanford-Binet, WPPSI, or WISC). Model-fitting indicated (1) a bidirectional relationship between MD and both persistence and approach: each variable impacted subsequent changes in the other and (2) a unidirectional relationship from MD to subsequent approach behavior. Twin analysis indicated genetic variance in MD was associated with subsequent genetic variance in all 3 factors. Thus, results indicate that growth in cognitive abilities may contribute to development of the ability to stay on task, adapt to new situations, and remain calm. Persistence and approach may contribute to development of cognitive abilities.

Grant Support: National Institute of Aging 1R03AG048850-01 \& R01AG063949-01.

\section{Genetic correlations between executive functions and intelligence in the adolescent brain cognitive development (ABCD) study}

Samantha M. Freis ${ }^{1,2}$, Claire L. Morrison ${ }^{1,2}$, Jeffrey M. Lessem ${ }^{1}$, John K. Hewitt ${ }^{1,2}$, Naomi P. Friedman ${ }^{1,2}$.

${ }^{1}$ Institute for Behavioral Genetics, University of Colorado Boulder ${ }^{2}$ Department of Psychology and Neuroscience, University of Colorado Boulder

Keywords Executive Control, Working Memory, IQ, Heritability

Executive functions (EFs), higher-order cognitive processes that enable control over thoughts and actions during goal-directed behavior, are phenotypically related to intelligence (IQ), and both are heritable across the lifespan. Existing research from twin studies has demonstrated genetic correlations between EFs and IQ. However, the magnitude of these correlations varies across samples, particularly between age groups, which suggests that the genetic correlations between these cognitive abilities may be more robust in childhood than in adulthood. We analyzed data from the large and representative Adolescent Brain Cognitive Development (ABCD) study, which comprised 11,875 children, including 749 twin pairs ( $M$ age = $9.91, S D=.62,48 \%$ female), to examine the phenotypic and genetic relationships between EFs and IQ in middle childhood. We identified two EF factors-Common EF and Updating-Specific. Both EFs are strongly related to IQ ( $r \mathrm{~s}=.64-.81)$, and both Common EF and IQ are heritable (53-67\%). We found a genetic correlation for Common EF and IQ $(r G=.86)$ that was not significantly different from 1 , but we did not find a significant genetic correlation for Updating-Specific ability and IQ. Overall, these results suggest that in middle childhood, EFs and IQ are phenotypically distinguishable; however, Common EF and IQ are closely related at the genetic level.

Grant Support: This research was supported by NIH Grants DA041120, MH063207, MH016880, and DA046413, Data used in the preparation of this presentation were obtained from the Adolescent Brain Cognitive Development (ABCD) Study ( https://abcdstudy.org), held in the NIMH Data Archive (NDA). This is a multisite, longitudinal study designed to recruit more than 10,000 children age 9-10 and follow them over 10 years into early adulthood. The ABCD Study is supported by the National Institutes of Health and additional federal partners under award numbers U01DA041022, U01DA041028, U01DA041048, U01DA041089, U01DA041106, U01DA041117, U01DA041120, U01DA041134, U01DA041148, U01DA041156, U01DA041174, U24DA041123, U24DA041147, U01DA041093, and U01DA041025. A full list of supporters is available at https://abcdstudy.org/federal-partners.html. A listing of participating sites and a complete listing of the study investigators can be found at https://abcdstudy.org/scientists/workgroups/. ABCD consortium investigators designed and implemented the study and/or provided data but did not necessarily participate in analysis or writing of this report. This manuscript reflects the views of the authors and may not reflect the opinions or views of the $\mathrm{NIH}$ or $\mathrm{ABCD}$ consortium investigators.

The $\mathrm{ABCD}$ data repository grows and changes over time. The $\mathrm{ABCD}$ data used in this report came from $A B C D$ release 2.0 (DOI: 10.15154/ 1503209).

\section{Investigating heterogenous genetic effects contributing to smoking behavior using the UK Biobank}

Scott A Funkhouser, Luke M Evans

Institute for Behavioral Genetics, University of Colorado, Boulder, Colorado, USA

Keywords Smoking, Gene-By-Moderator Interactions, Mood Disorders, Heterogeneity, GWAS

Tobacco smoking imposes a heterogenous burden within a population, disproportionally affecting certain groups of individuals while having contributed to millions of preventable deaths. Heterogenous tobacco usage is evident from longitudinal studies, which have suggested those with mood disorders such as anxiety and depression are more likely to later become nicotine-dependent smokers. Differing tobacco usage is furthermore seen between late-onset smokers compared to early-onset smokers, and between sexes. To partially explain these observations, family studies have implicated heterogenous genetic effects for numerous smoking behaviors, but little work using genetic marker data has been performed to support these findings. Here, we use the UK Biobank to infer heterogenous genetic effects for smoking heaviness using cigarettes per day (CPD) records, smoking 
initiation (SI), and smoking cessation (SC).We observed weak but suggestive evidence of disproportional SNP effects for CPD between MDD DSMV-like cases and controls $\left(\hat{r}_{g}=0.69, \mathrm{SE}=0.15, p\right.$-value $=$ 0.047) and discover SNPs with MDD-dependent effects reaching genome-wide significance (GWS; $p$-value $<5 \times 10^{-8}$ ) near GYPA, a gene previously implicated in COPD. We furthermore observed strong evidence for disproportional genetic effects for SI between sexes $\left(\hat{r}_{g}=0.82, \mathrm{SE}=0.02, p\right.$-value $\left.=4.22 \times 10^{-9}\right)$. Curiously, we observe no evidence for differing sex-specific SNP effects for SI at GWS but instead find 23 independent loci that reach GWS in one sex without reaching nominal significance $(p$-value $<0.05)$ in the opposite sex. This work suggests that the genetic determinants for smoking initiation are sex-dependent and discovers novel SI-associated loci using sex-stratified genome-wide association.

Grant Support: NIMH T32, Grant 5T32MH016880-38.

\section{The intensity of formal daycare attendance decreases the shared environment contribution to school readiness}

${ }^{1}$ Éloi Gagnon, ${ }^{1}$ Michel Boivin, ${ }^{1}$ Geneviève Morneau-Vaillancourt, ${ }^{2}$ Mara Brendgen, ${ }^{3}$ Frank Vitaro, ${ }^{1}$ Ginette Dionne.

${ }^{1}$ Department of Psychology, Laval University, Quebec, Quebec, Canada

${ }^{2}$ School of psychoeducation, Quebec University in Montreal, Montreal, Quebec, Canada

${ }^{3}$ Department of Psychology, University of Montreal, Montreal, Quebec, Canada

Keywords Daycare, School readiness, $\mathrm{G} \times \mathrm{E}$ interaction, Twin study,

Context It is unclear if daycare attendance could moderate genetic and environmental contribution to school readiness.

Sample Prospective, population-based and genetically descriptive sample of 660 pairs of French Canadian twins (QNTS).

Method We computed moderation models of $\mathrm{G} \times \mathrm{E}, \mathrm{CxE}$ and ExE (Purcell 2002) to assess how daycare attendance moderates genetic, shared environment and unique environment contributions to school readiness. The number of hours per week of formal daycare attendance was reported by the primary caregiver at 5, 18, 36 and 48 months. We calculated a single score of hours per week by averaging the four-time points. School readiness was assessed with the Lollipop test at 60 months.

Phenotypic results After correction for 7 relevant socioeconomic and perinatal covariates, each additional hour per week of formal daycare attendance is associated with a $0.0195 \%$ CI $(0.00-0.02)$ standard deviation increase on the Lollipop test $(\mathrm{p}=.02)$.

Genetic results The best fitting moderation model shows that daycare attendance moderates the $\mathrm{C}$ contribution but not $\mathrm{A}$ and $\mathrm{E}$ to individual differences in school readiness. The model shows that $\mathrm{C}$ explains $48 \%$ of individual differences in school readiness for children not attending formal daycare, and decreased gradually to $18 \%$ for children attending formal daycare full time, e.g., 40 hours per week.

Conclusion For school readiness, formal daycare settings appear to act as normalizing environments, possibly buffering the liability stemming from less stimulating home environment during the preschool years.

\section{References}

Purcell, S. (2002). Variance Components Models for Gene-Environment Interaction in Twin Analysis. Twin Research, 5(06), 554-571. doi:10.1375/136905202762342026
Grant Support: The Social Sciences and Humanities Research Council of Canada (SSHRC).

\section{Fitting problems: evaluating model fit in behavior genetic models}

S. Mason Garrison ${ }^{1,2}$, Joseph Lee Rodgers ${ }^{2}$

${ }^{1}$ Department of Psychology, Wake Forest University Winston-Salem, North Carolina, USA

${ }^{2}$ Department of Psychology and Human Development, Peabody College, Vanderbilt University, Nashville, Tennessee, USA

Keywords Methods; Structural Equation Modeling; Best Practices; Model Selection

In behavior genetics, like many fields, researchers must decide whether their models adequately explain their data-whether their models "fit" at some satisfactory level. Well-fitting models are compelling, whereas poorly-fitting models are not (Rodgers and Rowe 2002). Oftentimes, researchers evaluate model fit by employing "universal" rules of thumb (e.g., Hu and Bentler 1999). However, these rules are not universal, and are-in fact-model specific (Kang et al. 2016).

Accordingly, I focused on developing fit criteria emulating $\mathrm{Hu}$ and Bentler (1999) for classic univariate models (ACE; CE; AE) by fitting simulated twin data to correctly- and incorrectly-specified models. Ideal criteria should consistently accept correct models and reject incorrect models. Classic ACE models were indistinguishable and virtually all fit indices were non-informative because (or especially when) they are saturated models. For non-ACE models, criteria were informative. Nevertheless, every metric employed, except TLI differed markedly across models and/or conditions. Universal solutions remain elusive, but promising approaches include nested model comparisons, increasing degrees of freedom, and ruthless skepticism.

\section{References}

Li-Tze \& Bentler (1999) Cutoff criteria for fit indexes in covariance structure analysis: Conventional criteria versus new alternatives, Structural Equation Modeling, 6:1, 1-55.

Rodgers \& Rowe (2002). Theory development should begin (but not end) with good empirical fits: A comment on Roberts and Pashler (2000). Psychological Review, 109(3), 599.

Kang, McNeish, \& Hancock (2016). The role of measurement quality on practical guidelines for assessing measurement and structural invariance. Educational and Psychological Measurement, 76(4), 533-561.

\section{Remember this: harmonization of episodic memory measures across twin studies of aging}

Margaret Gatz ${ }^{1}$, Susan E. Luczak ${ }^{2}$, Shandell Pahlen ${ }^{3}$, Teresa Lee ${ }^{4}$, Marianne Nygaard ${ }^{5}$, Matthew S. Panizzon ${ }^{6}$, Brenda L. Plassman ${ }^{7}$, Chandra A. Reynolds ${ }^{3}$, Keith E. Whitfield ${ }^{8}$, for the IGEMS Consortium

${ }^{1}$ Center for Economic and Social Research, University of Southern California, Los Angeles, California, USA;

${ }^{2}$ Department of Psychology, University of Southern California, Los Angeles, California, USA; ${ }^{3}$ Department of Psychology, University of California, Riverside, Riverside, California, USA;

${ }^{4}$ Centre for Healthy Brain Ageing, School of Psychiatry, Faculty of Medicine, UNSW, Sydney, Australia; 
${ }^{5}$ Department of Public Health, University of Southern Denmark, Odense, Denmark;

${ }^{6}$ Department of Psychiatry, University of California, San Diego, La Jolla, California, USA;

${ }^{7}$ Department of Psychiatry \& Behavioral Sciences, Duke University School of Medicine, Durham, North Carolina, USA;

${ }^{8}$ Office of the Provost, Wayne State University, Detroit, Michigan, USA

\section{Keywords Episodic memory; twin studies}

Episodic memory impairment is a hallmark symptom of dementia and concern among individuals as they grow older. In the Interplay of Genes and Environment in Multiple Studies (IGEMS) consortium, most studies measured episodic memory by giving the participant a list of words to learn. However, different studies used different numbers of words, unrelated words or words within categories, different numbers of learning trials, different wait times before delayed recall, and face-to-face or telephone administration.

Data are from 12 IGEMS studies, from Sweden, Denmark, Australia, and the U.S. ( $\mathrm{N}=37,808$ individuals; 11,995 complete twin pairs; mean age $=63.6,95 \%$ between 45 and 80 ). We selected the only variable consistent across studies: the first time that the participant repeated back the list of words. From raw scores, within each IGEMS study, we calculated percent of words correctly recalled, then created a $\mathrm{T}$ score standardized to mean $=50, \mathrm{SD}=10$ for noncognitively impaired individuals aged 65-69.9. We applied this formula to all individuals in the respective study at all waves.

Cross-sectional phenotypic analyses found expected age differences, with lower scores at older ages. Heritability of memory was higher in younger aged twins compared to older ages $\left(\mathrm{a}^{2}{ }_{40}=.29 ; \mathrm{a}^{2}{ }_{70}\right.$ $=.12$ ).

Discussion: This report adds episodic memory to available harmonized cognitive variables in IGEMS. Compared to previous results for other cognitive abilities in IGEMS studies, the findings are similar to the pattern reported for digit span, but differs from verbal abilities where heritability increased with age.

Grant Support: NIH Grant Nos. R01 AG060470 and R01 AG059329.

\section{The Flynn Effect across three WISC versions and four decades in a single sample of U.S. children}

Evan J. Giangrande ${ }^{1}$, Christopher R. Beam ${ }^{2,3,4}$, Deborah W. Davis ${ }^{5}$, Eric Turkheimer ${ }^{1}$

${ }^{1}$ Department of Psychology, University of Virginia, Charlottesville, Virginia, USA

${ }^{2}$ Department of Psychology, University of Southern California, Los Angeles, California, USA

${ }^{3}$ School of Gerontology, University of Southern California, Los Angeles, California, USA

${ }^{4}$ Center for the Changing Family, University of Southern California, Los Angeles, California, USA

${ }^{5}$ Department of Pediatrics, University of Louisville, Louisville, Kentucky, USA

\section{Keywords Flynn Effect; intelligence; g; Louisville Twin Study}

The secular rise in IQ scores over time, known as the Flynn Effect, is typically documented by observing increasing mean scores in a sample across repeated assessments using different test versions. Other evidence comes from studies of military conscripts, wherein cohorts from more recent generations scored systematically higher than previous cohorts on the same test version. Each of these approaches have distinct strengths and limitations. Results of previous studies suggest that the strength of the Flynn Effect fluctuates across cognitive domains, leading some to question whether rises in cognitive ability scores reflect genuine increases in general intelligence $(g)$.

In this study, we examined the Flynn Effect across late childhood and early adolescence (ages 7-15 years) in data from the Louisville Twin Study. Three versions of the WISC were administered over 40 years of data collection (1957-1999), generating comprehensive, longitudinal subtest data. This unique data structure enabled us to test for both test version and cohort effects simultaneously in a single sample, which had not been done previously. Using three SEM models (latent growth curve; longitudinal multiple indicators, multiple causes (MIMIC); exploratory bi-factor MIMIC), we observed the Flynn Effect as decreases in mean performance upon test re-standardization, and as gains in mean performance across generational cohorts. Flynn Effect magnitude varied across subtests in a manner that was not strictly proportional to subtests' $g$-loadings. We argue that these gains reflect important changes in intelligence, regardless of whether or not they are driven by gains in $g$.

Grant Support: National Institute on Aging Grant Nos. R03AG048850-01 and R01AG063949-01.

\section{The genetic and environmental components of economic behavior}

Alexandros Giannelis ${ }^{1}$, Scott Vrieze ${ }^{1}$, Matt McGue ${ }^{1}$, James J. Lee ${ }^{1}$, Robin Corley ${ }^{2}$, John Hewitt ${ }^{2}$, Christian Hopfer ${ }^{3}$

${ }^{1}$ Department of Psychology, University of Minnesota, Minneapolis, Minnesota, USA

${ }^{2}$ Institute of Behavioral Genetics, University of Colorado, Boulder, Colorado, USA

${ }^{3}$ Department of Psychiatry, University of Colorado, Denver, Colorado, USA

Keywords Income, intelligence, twins, genoeconomics

The emerging field of genoeconomics is attempting to answer perennial questions regarding the determinants of wealth accumulation and social inequality. A plethora of studies has demonstrated that genetic factors explain some of the variance in income, wealth and saving rates (Cronqvist and Siegel 2015; Hyytinen et al. 2019; Xu et al. 2017). Less studied has been the heritability of saving attitudes. Using data from the Colorado and Minnesota Twin Study (2214 twins) we estimate the heritability of various economic outcomes (labor income, family income, financial strain, financial distress), as well as self-reported beliefs about saving. Preliminary results suggest that genetic factors account for $37 \%$ to $50 \%$ of the variance in those economic outcomes, while saving attitude shows negligible heritability. All outcomes associate significantly with intelligence and educational attainment polygenic scores. Saving attitude is strongly predictive of all the aforementioned economic outcomes. This effect is not mediated by intelligence or personality traits such as impulsiveness or irresponsibility. The evidence suggests that there is a component of economic behavior which is distinct from intelligence and personality, and more related to time preference. This factor is not significantly heritable and is predictive of economic success.

\section{References}

Cronqvist, H., \& Siegel, S. (2015). The Origins of Savings Behavior. Journal of Political Economy, 123(1), 123-169. https://doi.org/ 10.1086/679284

Hyytinen, A., Ilmakunnas, P., Johansson, E., \& Toivanen, O. (2019). Heritability of lifetime earnings. The Journal of Economic Inequality, 17(3), 319-335. https://doi.org/10.1007/s10888-019-09413-x 
Xu, Y., Briley, D. A., Brown, J. R., \& Roberts, B. W. (2017). Genetic and environmental influences on household financial distress. Journal of Economic Behavior \& Organization, 142, 404-424. https://doi.org/10.1016/j.jebo.2017.08.001

Grant Support: Grant Number: R01 DA042755 from the U.S. National Institute on Drug Abuse.

\section{Assortative mating for educational attainment in Finnish and Dutch twins and their spouses}

\author{
B.M.A. Gonggrijp ${ }^{1}$, K. Silventoinen ${ }^{2,3}$, C.V. Dolan ${ }^{1}$, J. Kaprio ${ }^{3,4}$, G. \\ Willemsen ${ }^{1,5}$ \\ ${ }^{1}$ Department of Biological Psychology, Vrije Universiteit Amster- \\ dam, Amsterdam, the Netherlands \\ ${ }^{2}$ Department of Social Research, University of Helsinki, Helsinki, \\ Finland \\ ${ }^{3}$ Department of Public Health, University of Helsinki, Finland. \\ ${ }^{4}$ Institute for Molecular Medicine Finland (FIMM), University of \\ Helsinki, Helsinki, Finland \\ ${ }^{5}$ Amsterdam Public Health Research Institute, Vrije Universiteit \\ Amsterdam, Amsterdam, the Netherlands
}

Keywords Assortative Mating, Education, Twins, Social Homogamy, Phenotypic Assortment

Assortative mating is the tendency to choose a partner with similar characteristics. When assortative mating is present for education it can have important societal consequences since it may lead to widening socio-economic inequalities. Often data are limited to spousal correlations, making it difficult to distinguish between the different causal mechanisms of assortative mating. Using information on monozygotic (MZ) and dizygotic (DZ) twins and their spouses can provide insight into the study of the mechanisms behind the spousal correlations.

The aim of this study is to understand the underlying mechanisms of assortative mating on educational attainment using large twin cohorts from two countries with similar schooling systems.

Methods: An extended twin model in which phenotypic assortment and social homogamy were modelled by delta paths was applied to data from the Finnish Twin Cohort (FTC) and the Netherlands Twin Register (NTR).

Dropping either phenotypic assortment or social homogamy from the model led to a significant decrease in model fit in both cohorts, indicating that both factors play a role in the non-random mating for educational attainment. Genetic, shared environmental and nonshared environmental influences accounted for 55\%,29\% and $16 \%$ of the variance in educational attainment in the Finnish cohort, and for $66 \%, 21 \%$ and $13 \%$ in the Dutch cohort, respectively. Phenotypic assortment contributed .35 in the FTC and .30 in the NTR to the spousal correlation, while social homogamy contributed .16 and .15 , respectively. This suggests that phenotypic assortment contributes more to the similarity of spouses than social homogamy.

\section{Causal mechanisms underlying parental influences on adolescent substance use}

Shelley A. Gresko ${ }^{1,2}$, Maya Rieselbach ${ }^{1,2}$, Soo Hyun Rhee ${ }^{1,2}$ ${ }^{1}$ Department of Psychology and Neuroscience, University of Colorado Boulder, Boulder, CO, USA

${ }^{2}$ Institute for Behavioral Genetics, University of Colorado Boulder, Boulder, CO, USA
Keywords adolescents, substance use, parenting, parent-adolescent relationships, adoption studies

Adolescent substance use (SU) is a pressing public health issue that can lead to later SU disorders and other detrimental outcomes. Genetically informed research indicates a significant contribution of shared environmental influences on adolescent SU (Hopfer et al. 2003). Putative environmental influences on adolescent SU include parental SU, parenting, and parent-adolescent relationship quality. There is mixed evidence that parental SU moderates associations between parenting and adolescent SU and that parenting mediates associations between parental and adolescent SU.

The present study will examine the putative environmental predictors of adolescent SU, using data from the Colorado Adoption Project. Data from adoptive and non-adoptive families will be examined to distinguish whether the associations between potential predictors and adolescent SU are due to environmental mediation and/ or passive $r$ GE. We will examine whether the magnitude of influence of parenting on adolescent SU is moderated by parental SU, and whether associations between adolescent and parental SU are mediated by parenting and parent-adolescent relationship quality.

\section{References}

Hopfer, C. J., Crowley, T. J., \& Hewitt, J. K. (2003). Review of Twin and Adoption Studies of Adolescent Substance Use. Journal of the American Academy of Child \& Adolescent Psychiatry, 42(6), 710-719. https://doi.org/10.1097/01.CHI.0000046848.56865.54

Grant Support: DA011015, HD010333, AG046938, DA017637.

\section{Integrative multi-omics analysis of genomic, epigenomic, and metabolomic data for childhood aggressive behavior}

Fiona A. Hagenbeek ${ }^{1,2}$, Jenny van Dongen ${ }^{1,2}$, René Pool ${ }^{1,2}$, Peter J. Roetman $^{3}$, Amy C. Harms ${ }^{4,5}$, Jouke Jan Hottenga ${ }^{1}$, Cornelis Kluft ${ }^{6}$, Olivier F. Colins ${ }^{3,7}$, Catharina E.M. van Beijsterveldt ${ }^{1}$, Marjolein M. L. J. Z. Vandenbosch ${ }^{1}$, Eveline L. de Zeeuw ${ }^{1,2}$, Simone Talens ${ }^{6}$, Vassilios Fanos ${ }^{8}$, Erik A. Ehli ${ }^{9}$, Gareth E. Davies ${ }^{9}$, Robert R. J. M. Vermeiren $^{3}$, Thomas Hankemeier ${ }^{4,5}$, Meike Bartels ${ }^{1,2,10}$, Sébastien Déjean $^{11}$, Dorret I. Boomsma ${ }^{1,2,10}$

${ }^{1}$ Department of Biological Psychology, Vrije Universiteit Amsterdam, Amsterdam, the Netherlands

${ }^{2}$ Amsterdam Public Health research institute, Amsterdam, the Netherlands

${ }^{3}$ Curium-LUMC, Department of Child and Adolescent Psychiatry, Leiden University Medical Center, Leiden, The Netherlands

${ }^{4}$ Division of Analytical Biosciences, Leiden Academic Center for Drug Research, Leiden University, Leiden, the Netherlands

${ }^{5}$ The Netherlands Metabolomics Centre, Leiden, The Netherlands

${ }^{6}$ Good Biomarker Sciences, Leiden, the Netherlands

${ }^{7}$ Department Special Needs Education, Ghent University, Ghent, Belgium

${ }^{8}$ Department of Surgical Sciences, University of Cagliari and Neonatal Intensive Care Unit, Cagliari, Italy

${ }^{9}$ Avera Institute for Human Genetics, Sioux Falls, South Dakota, USA

${ }^{10}$ Amsterdam Neuroscience, Amsterdam, the Netherlands

${ }^{11}$ Toulouse Mathematics Institute, University of Toulouse, CNRS, Toulouse, France

Keywords Multi-Omics, Aggressive Behavior, Genomics, Epigenomics, Metabolomics 
Multi-omics studies allow for biomarker identification for improved patient stratification and elucidation of underlying disease-associated biological processes. Here, we present an integrated multi-omics analysis for childhood aggression (AGG) based on genomics, epigenomics and metabolomics data. Urine and buccal-cell samples were collected in a cohort of twins ( $\mathrm{N}=798$ individuals, mean age $=9.5$, males $=52 \%$, cases $=42.2 \%$ ), recruited from the Netherlands Twin Register, to assess urinary metabolites, genome-wide buccal methylation markers and genotypes. Polygenic scores (PGS) were calculated for AGG (Ip et al. 2019), Attention-Deficit/Hyperactivity Disorder (Demontis et al. 2019) and Major Depressive Disorder (MDD) (Wray et al. 2018). The multi-omics data was integrated with multi-block Partial Least Squares Discriminant Analysis (Singh et al. 2019). The performance and predictive ability of this model was evaluated in a holdout subset of the data. The multi-omics model, including the PGSs for AGG and MDD, 15 metabolites and 30 methylation markers, had poor predictive ability (AUC $=0.42-0.55$ ). Congruent with previous research (Hagenbeek et al. 2020), our model included metabolites involved in cysteine metabolism, oxidative stress and neurotransmitter pathways. The methylation markers were predominantly located in $\mathrm{CpG}$ islands, previously mainly associated with neurodevelopmental presentations and congenital anomalies and chiefly enriched for the postsynaptic membrane. We will extend this model with PGSs of additional AGG-related traits and evaluate the effect of genetic nurture (Kong et al. 2018). Clinical validation of the multi-omics model will be appraised in a sample of children referred to a youth psychiatric clinic (Curium-LUMC). Overall, our multiomics model highlights potential biological mechanisms for childhood AGG.

\section{References}

Demontis, D., Walters, R. K., Martin, J., Mattheisen, M., Als, T. D., Agerbo, E., ... Neale, B. M. (2019). Discovery of the first genomewide significant risk loci for attention deficit/hyperactivity disorder. Nature Genetics, 51(1), 63-75. https://doi.org/10.1038/s41588-018-0269-7

Hagenbeek, F. A., Roetman, P. J., Pool, R., Kluft, C., Harms, A. C., van Dongen, J., ... Boomsma, D. I. (2020). Urinary Amine and Organic Acid Metabolites Evaluated as Markers for Childhood Aggression: The ACTION Biomarker Study. Frontiers in Psychiatry, 11(165). https://doi.org/10.3389/fpsyt.2020.00165

Ip, H. F., Laan, C. M. van der, Brikell, I., Sánchez-Mora, C., Nolte, I. M., Pourcain, B. S., ... Boomsma, D. I. (2019). Genetic Association Study of Childhood Aggression across raters, instruments and age. BioRxiv. https://doi.org/10.1101/854927

Kong, A., Thorleifsson, G., Frigge, M. L., Vilhjalmsson, B. J., Young, A. I., Thorgeirsson, T. E., ... Stefansson, K. (2018). The nature of nurture: Effects of parental genotypes. Science, 359(6374), 424-428. https://doi.org/10.1126/science.aan6877

Singh, A., Shannon, C. P., Gautier, B., Rohart, F., Vacher, M., Tebbutt, S. J., \& Cao, K. A. L. (2019). DIABLO: An integrative approach for identifying key molecular drivers from multi-omics assays. Bioinformatics, 35(17), 3055-3062. https://doi.org/10.1093/bioinformatics/bty 1054

Wray, N. R., Ripke, S., Mattheisen, M., Trzaskowski, M., Byrne, E. M., Abdellaoui, A., ... Sullivan, P. F. (2018). Genome-wide association analyses identify 44 risk variants and refine the genetic architecture of major depression. Nature Genetics, 50(5), 668-681. https://doi.org/10.1038/s41588-018-0090-3

Grant Support: "Aggression in Children: Unraveling gene-environment interplay to inform Treatment and InterventiON strategies" project (ACTION; European Union Seventh Framework Program under grant agreement no 602768). The Netherlands Twin Register is supported by grant NWO 480-15-001/674: Netherlands Twin Registry Repository and by multiple grants from the Netherlands Organization for Scientific Research (NWO). MB is supported by an ERC consolidator grant (WELL-BEING 771057 PI Bartels). JvD is supported by the NWO-funded X-omics project (184.034.019).

\section{Toddler temperament modestly predicts middle childhood mental health profiles, via genetic pathways}

\section{Emily C. Hilton ${ }^{1}$ and H. Hill Goldsmith ${ }^{1}$}

${ }^{1}$ University of Wisconsin - Madison Department of Psychology and Waisman Center, Madison, Wisconsin, USA

Keywords developmental psychopathology; temperament; twin study

Temperament provides a promising avenue for understanding childhood psychopathology risk, yet most studies only examine temperament links to single disorders. In contrast, we examine how broad temperament factors during toddlerhood predict empiricallyderived classes of psychopathology symptoms in middle childhood.

Latent class analysis of symptoms at age 7 yielded 9 classes for each gender in a birth record-based twin sample (Vendlinski et al. 2014) Classes revealed pervasive symptom overlap. Using parent report on the Toddler Behavior Assessment Questionnaire $(\mathrm{N}=$ 1037), a linear mixed effects model with nesting within families showed the temperament factors were generally modestly but highly significantly associated with symptom classes. The low interest/attention and high negative affect/low control temperament factors predicted membership in more impaired/severe classes, which contained both internalizing and externalizing symptoms. Lower temperamental fear in toddlerhood predicted classes with moderate impulsivity. High interest/attention and low negative affect/high control predicted low and mild symptoms classes. Thus, toddler temperament factors were non-specific risk or protective factors for childhood symptoms. Bivariate Cholesky decompositions indicated that for the boys' low symptom class, the proportion of variance accounted for by genetic effects of high negative affect/low control was .35 , but only .06 for a high symptom class with ADHD/conduct disorder features. For the same symptom classes, genetic effects attributable to the interest/attention factor were only .04 and .02 . Genetic temperament symptoms paths were variable, with protective temperament factors showing greater genetic mediation.

\section{References}

Vendlinski, MK et al., (2014). Relative Influence of Genetics and Shared Environment on Child Mental Health Symptoms Depends on Comorbidity. PLoS ONE, 9(7).

Grant Support: Acknowledgements: Data collection for this project was supported by R37 MH050560 and R01 MH059785 from the National Institute of Mental Health. The writing of this manuscript was partly supported by K01MH113710, T32 MH018931, P50 MH084051, and RO1 MH101504. Infrastructure support was provided by core grants to the Waisman Center from the National Institute of Child Health and Human Development (P30 HD003352, U54 HD09025). We thank the families who participated in the study and the staff members who helped with data collection.

\section{Do common and rare variants work together on the same gene to affect phenotype?}

\author{
Meng Huang ${ }^{1} \&$ Matthew C. Keller ${ }^{1}$ \\ ${ }^{1}$ Institute for Behavioral Genetics, University of Colorado Boulder, \\ Boulder, Colorado, USA
}


Keywords Rare variants; GWAS; Gene effect; Human genetics.

More and more evidence indicate that the rare variants could explain a part of the genetic variance. However, how the common variants and rare variants work together to control the phenotype is still unclear. How many associated rare variants collocated on the same gene with associated common variants? Or the rare variants rarely overlapped with the common variants as they have their own mechanism to affect phenotype?

To answer these questions, several comparisons among different GWAS methods were implemented in two datasets. One dataset is the exome-sequenced UK biobank data, which was used for the GWAS of rare variants and includes 49,960 samples and 10,448,724 SNPs. Another dataset is the imputed UK biobank DNA array data, which was used for the GWAS of common variants and includes 486,565 samples and 89,716,503 SNPs. MAGMA, SKAT, and burden test were used in GWAS analysis. There were 6 phenotypes including height, BMI, diabetes, heel bone density, blood pressure, and basal metabolic rate were used in the comparison.

The results indicated that there is no significant correlation among 90 comparisons between different methods in 6 phenotypes. Although it is too early to state that the rare variants and common variants are not closely related in gene-level, this result could be the benchmark in the following research of the relationship of rare and common variants.

\section{Integrative multi-omics analysis of epigenomic and metabolomic data for attention-deficit/ hyperactivity disorder}

Nikki Hubers ${ }^{1}$, Fiona A. Hagenbeek ${ }^{1,2}$, Jenny van Dongen ${ }^{1,2}$, Sébastien Déjean ${ }^{11}$, Peter J. Roetman ${ }^{3}$, Amy C. Harms ${ }^{4,5}$, Jouke Jan Hottenga $^{1}$, Cornelis Kluft ${ }^{6}$, Olivier F. Colins ${ }^{3,7}$, Catharina E.M. van Beijsterveldt ${ }^{1}$, Marjolein M. L. J. Z. Vandenbosch ${ }^{1}$, Eveline L. de Zeeuw $^{1,2}$, Simone Talens ${ }^{6}$, Vassilios Fanos ${ }^{8}$, Erik A. Ehli ${ }^{9}$, Gareth E. Davies $^{9}$, Robert R. J. M. Vermeiren ${ }^{3}$, Thomas Hankemeier ${ }^{4,5}$, Meike Bartels ${ }^{1,2,10}$, René Pool ${ }^{1,2}$, Dorret I. Boomsma ${ }^{1,2,10}$

${ }^{1}$ Department of Biological Psychology, Vrije Universiteit Amsterdam, Amsterdam, the Netherlands

${ }^{2}$ Amsterdam Public Health research institute, Amsterdam, the Netherlands

${ }^{3}$ Curium-LUMC, Department of Child and Adolescent Psychiatry, Leiden University Medical Center, Leiden, The Netherlands

${ }^{4}$ Division of Analytical Biosciences, Leiden Academic Center for Drug Research, Leiden University, Leiden, the Netherlands

${ }^{5}$ The Netherlands Metabolomics Centre, Leiden, The Netherlands;

${ }^{6}$ Good Biomarker Sciences, Leiden, the Netherlands

${ }^{7}$ Department Special Needs Education, Ghent University, Ghent, Belgium

${ }^{8}$ Department of Surgical Sciences, University of Cagliari and Neonatal Intensive Care Unit, Cagliari, Italy

${ }^{9}$ Avera Institute for Human Genetics, Sioux Falls, South Dakota, USA

${ }^{10}$ Amsterdam Neuroscience, Amsterdam, the Netherlands

${ }^{11}$ Toulouse Mathematics Institute, University of Toulouse, CNRS, Toulouse, France

Keywords Multi-Omics, Attention-Deficit/Hyperactivity Disorder, Epigenomics, Metabolomics, Twins

Attention-deficit/hyperactivity disorder (ADHD) is a complex trait with a substantial heritability with other 'omics' e.g. epigenomics and metabolomics, influences on ADHD beginning to be explored. Multiomics is an evolving field which combines data from multiple 'omics' levels and provides methods for their simultaneous analyses to identify biological mechanisms and biomarkers for complex traits. This project applies an integrative multi-omics method, called DIABLO, based on multi-block Partial Least Squares Discriminant Analysis (PLS-DA) to build a predictive model for ADHD from metabolomics and epigenomics data.

First-morning urine and buccal-cell samples were collected from 606 twins $(\mathrm{N}$ complete pairs $=275$, mean age $=9.3[\mathrm{SD}=0.63]$, male $=54.9 \%$ ) who took part in Netherlands Twin Register ACTION project (Aggression in Children: Unraveling gene-environment interplay to inform Treatment and InterventiON strategies).

Dichotomous ADHD status was based on the mother-rated Revised Conners' Parent Rating Scale (CPRS-R). First, in 20\% of the data, the discriminative capacities of each 'omics' level and pair-wise cross-omics relationships were analysed with PLS-DA and PLS, respectively. Next, multi-block PLS-DA was applied to $50 \%$ of the data. The resulting model was used to predict the ADHD status in the remaining $30 \%$ of the data. The model selected metabolites and methylation probes involved in dopaminergic pathways and oxidative stress, mechanisms that have previously been associated with ADHD. However, the predictive accuracy of the model was relatively low (Area under the curve $=0.449-0.588, \mathrm{p}>0.10$ ). Overall, the model seems promising and underlines the potential of a multi-omics approach in complex traits such as ADHD.

Grant Support: "Aggression in Children: Unraveling gene-environment interplay to inform Treatment and InterventiON strategies" (ACTION; European Union Seventh Framework Program under grant agreement no 602768). The Netherlands Twin Register is supported by grant NWO 480-15-001/674: Netherlands Twin Registry Repository and by multiple grants from the Netherlands Organization for Scientific Research (NWO) MB is supported by an ERC consolidator grant (WELL-BEING 771057 PI Bartels). JvD is supported by the NWO-funded X-omics project (184.034.019).

\section{Polygenic propensity for a psychiatric disorder is associated with nutrient intake}

Avina K. Hunjan ${ }^{1,2}$, Christopher Hübel ${ }^{1,3}$, Yuhao Lin ${ }^{1}$, Thalia C. Eley $^{1,2}$, Gerome Breen ${ }^{1,2}$.

${ }^{1}$ Social Genetic \& Developmental Psychiatry Centre, Institute of Psychiatry, Psychology \& Neuroscience, King's College London, UK ${ }^{2}$ NIHR Biomedical Research Centre for Mental Health; South London and Maudsley NHS Trust, UK

${ }^{3}$ Department of Medical Epidemiology and Biostatistics, Karolinska Institutet, Stockholm, Sweden

Keywords Nutrition, psychiatric disorders, polygenic scores.

Despite the observed association between psychiatric disorders and nutrient intake, genetic studies are limited. To gain a better insight into the relationship between psychiatric disorders and nutrient intake these studies are needed. We examined whether polygenic scores (PGS) for psychiatric disorders are associated with self-reported nutrient intake using data obtained by the UK Biobank diet questionnaire $(\mathrm{N}=163,619)$. Association was assessed using linear mixed effects models for the analysis of data with repeated measures. We find that PGSs for psychiatric disorders are differentially associated with nutrient intake, with attention-deficit/hyperactivity disorder, bipolar disorder and schizophrenia showing the strongest associations, whilst autism spectrum disorder showed the weakest. We found a higher anorexia nervosa PGS was associated with higher intake of fibre, folate, iron and vitamin $\mathrm{C}$; however, associations were predominantly driven by socioeconomic status and educational attainment. This was also true for obsessive-compulsive disorder PGS and fibre intake. A higher major depressive disorder PGS with higher 
alcohol intake but lower iron, vitamin $\mathrm{C}$ and vitamin $\mathrm{E}$ intake. Furthermore, a higher alcohol dependence PGS was associated with higher alcohol intake independent of socioeconomic status, and individuals with higher persistent thinness PGS reported their food to weigh less. Overall, these findings suggest that polygenic propensity for a psychiatric disorder is associated with dietary behaviour. The nutrient intake is based on self-reported data and findings must be mindfully interpreted given its inherent limitations.

\section{Interaction between polygenic risks of obesity related traits and lifestyle in the UK Biobank}

\author{
Liang-Dar Hwang ${ }^{1}$, Shannon D’Urso ${ }^{1}$ \\ ${ }^{1}$ The University of Queensland Diamantina Institute, The University \\ of Queensland, Brisbane, Queensland, Australia
}

Keywords Polygenic risk score, BMI, obesity, lifestyle, interaction.

Obesity-related health conditions have well-documented lifestyle and genetic components. There is growing interest in translational applications of polygenic risk scores (PRS) for risk prediction of obesityrelated conditions, however it remains unclear whether PRS can be used for prioritizing behavioural change interventions. We constructed PRS of six common obesity risk factors (body mass index [BMI], high-density lipoprotein cholesterol [HDL], low-density lipoprotein cholesterol [LDL], triglycerides [TG], systolic blood pressure [SBP], diastolic blood pressure [DBP]) in 382,376 unrelated Europeans in the UK Biobank. We tested interactions between these PRS and four lifestyle traits (meat-based diet, physical activity, sedentary time, sleep duration) using linear regression models (e.g. $\mathrm{BMI} \sim \mathrm{PRS}_{\mathrm{BMI}}+$ meat-diet $+\mathrm{PRS}_{\mathrm{BMI}} \times$ meat-diet $)$. We found nine interactions $\left(\mathrm{PRS}_{\mathrm{BMI}}\right.$ with diet and physical activity, $\mathrm{PRS}_{\mathrm{HDL}}$ with physical activity and sedentary time, PRS $_{\mathrm{LDL}}$ with sedentary activity, $\mathrm{PRS}_{\mathrm{TG}}$ with diet and sedentary activity, $\mathrm{PRS}_{\mathrm{SBP}}$ with diet, and $\mathrm{PRS}_{\mathrm{DBP}}$ with diet; $\mathrm{P}<$ Bonferroni-corrected threshold of 0.002). In general, the effects of lifestyle on BMI were larger among participants at higher genetic risk of BMI and the effects on blood pressures were in the opposite direction; the effects on lipids varied. For example, BMI unit change per $\mathrm{z}$-score increase in meat-based diet was higher among participants in the top quartile of PRS BMI $_{\text {(beta }}$ $[95 \%$ confidence interval $]=0.748[0.710,0.786])$ than among those in the bottom quartile (beta [95\% confidence interval] $=0.644[0.609$, $0.680]$ ). Our findings suggest that behavioural change interventions may have different effects on obesity risk reduction depending on an individual's genetic risk.

\section{Genetic influence on academic achievement and educational opportunities controlled by socioeconomic effect}

\author{
Juko Ando ${ }^{1}$ \\ ${ }^{1}$ Faculty of Letters, Keio University, Minato-ku, Tokyo, Japan
}

Keywords Academic Achievement, Economic Disparity, Family Environment, Learning Opportunity, Twin Method

Numerous studies of educational sociology have been reporting that the parental socioeconomic status (SES) influences educational environment and academic achievement of children (eg. Broer, Bai and Froseca 2019). Although SES contains not only shared environmental but also genetic components, behavioral genetic studies revealed that even after controlling genetic influence, shared environmental factors substantially affect SES (eg. Walker et al. 2005). The current study investigated how much genetic factors influenced academic achievement and educational opportunities of children after controlling SES using the twin method.

2359 pairs of twins $(\mathrm{MZ}=689, \mathrm{DZ}=783$ for elementary school and $\mathrm{MZ}=511, \mathrm{DZ}=376$ for secondary school) and their parents in Tokyo and its neighbor prefectures provided parental income and educational history, nurturing attitude, number of books, childrens' academic achievements and their learning environments (study time and extracurricula activities).

Parental SES significantly correlated with children's academic achievement and their learning environment $\left(r_{\max }=.30\right)$. However there were still huge genetic variance for academic achievement especially in elementary school level (around 70\%) even after controlling SES. Genetic variation for academic achievement decreased in secondary school level. On the contrary, children's learning environment were exclusively affected byu shared environment for both levels of schools.

\section{References}

Broer, M., Bai, Y., \& Fonseca, F. (2019) Socioeconomic inequality and educational outcomes: Evidence from twenty years of TIMSS. IEA.

Walker, S.O., petrill, S.A., \& Plomin, R. (2005) A genetically sensitive investigation of the effects of the school environment and socioeconomic status on academic achievement in seven-year olds. Educational Psychology, 25(1), 55-73.

Grant Support: Brain Science and Education, Japan Science and Technology Agency 2003-2007

\section{Genetic and environmental overlap of agency and the big five personality traits}

Eleanor J. Junkins ${ }^{1}$, Daniel A. Briley ${ }^{1}$, Jaime Derringer ${ }^{1}$

${ }^{1}$ Department of Psychology, University of Illinois at Urbana-Champaign, Champaign, Illinois, USA

Keywords personality; agency; Big 5; twin study; heritability

Agency refers to an individual's drive to master their environment, to assert the self, to experience competence, achievement, and power. Agency has been shown to relate positively to psychological wellbeing and self-esteem (see Helgeson and Fritz 1999, Jo. of Res. in Personality). Further, Agency is correlated with several of the Big Five personality traits (Openness, Agreeableness, and Conscientiousness). The present study estimated the heritability of Agency and examined the extent to which the concurrent associations between the Big Five and Agency are mediated by genetic and environmental factors. We used data from wave 1 of the Midlife in the United States (MIDUS) twin sample ( $N=921$ pairs). We conducted a biometric decomposition of Agency, demonstrating substantial influence of both genetic and environmental factors on Agency. Next, we fit a multivariate model to estimate the extent to which there are shared genetic and environmental influences between the Big Five and Agency, as well as the residual genetic and environmental influences on Agency. The genetic correlations will be discussed under the scope of the phenotypic null hypothesis.

Grant Support: Daniel A. Briley was supported by a Jacobs Foundation Research Fellowship. 


\section{Predicting alcohol use from genome-wide polygenic scores, environmental factors, and their interactions}

Radhika Kandaswamy ${ }^{1}$, Andrea Allegrini ${ }^{1}$, Sophie Cave $^{2}$, Robert Plomin $^{1}$ and Sophie von Stumm ${ }^{2}$

${ }^{1}$ King's College London, Social, Genetic \& Developmental Psychiatry Centre, Institute of Psychiatry, Psychology \& Neuroscience, London, UK

${ }^{2}$ Department of Education, University of York, Heslington, York, UK

Keywords Polygenic, $\mathrm{G} \times \mathrm{E}$, Alcohol Use, AUDIT-C, Emerging Adulthood

Alcohol use in emerging adulthood is associated with adverse life outcomes but its risk factors are not well known. Genome-wide polygenic scores (GPS) based on a number of genome-wide association (GWA) studies for alcohol consumption have been able to predict alcohol use, although variance accounted for is rather low $(\sim$ 2.5\%) (Liu et al. 2019). Identifying environments that help reducing alcohol consumption among people with greater genetic propensities for alcohol use is important for developing effective interventions. Here, we tested if genetic influences on alcohol consumption change across environmental contexts, i.e., gene-environment $(G \times E)$ interactions.

We predicted alcohol use in 3153 adults aged 22 years from the Twins Early Development Study (TEDS) using (a) GPS based on genome-wide association studies for the target phenotypes number of drinks per week [1] and Alcohol Use Disorders Identification Test (AUDIT-C) scores (Sanchez-Roige et al. 2019), (b) 30 environmental factors, and (c) their interactions (i.e. $G \times E$ effects).

The GPS accounted for up to $1.9 \%$ of the variance in alcohol use (i.e. AUDIT-C score), while the 30 measures of environmental factors together accounted for $21 \%$. The 30 GPS-environment interaction terms accounted for an additional $0.3 \%$ variance, but none of the interactions survived multiple testing threshold.

We found that both genetic and environmental factors predict alcohol use in young adults, but we did not find support for $\mathrm{G} \times \mathrm{E}$ interaction effects on alcohol use. Our findings suggest that GPS and environmental factors have primarily direct, additive effects rather than interacting systematically.

References

Liu, M., et al., Association studies of up to 1.2 million individuals yield new insights into the genetic etiology of tobacco and alcohol use. Nat Genet, 2019. 51(2): p. 237-244.

Sanchez-Roige, S., et al., Genome-Wide Association Study MetaAnalysis of the Alcohol Use Disorders Identification Test (AUDIT) in Two Population-Based Cohorts. Am J Psychiatry, 2019. 176(2): p. $107-118$.

Grant Support: This study was supported by the European Foundation for Alcohol Research (ERAB) (EA 18 16, SvS \& RP) and Jacobs Foundation (Early Career Fellowship 2017-2019, SvS). TEDS is supported by a program grant from the UK Medical Research Council (grant G0901245 and previously G0500079, RP), with additional support from the US National Institutes of Health (grants HD044454 \& HD059215, RP)
The role of oxytocin receptor gene polymorphisms in depression-related traits: gene-environment interactions

Anastasiya Kazantseva ${ }^{1}$, Yuliya Davydova ${ }^{1}$, Marina Lobaskova ${ }^{3}$, Renata Enikeeva ${ }^{1}$, Rustam Mustafin ${ }^{2}$, Sergey Malykh $^{3}$, Elza Khusnutdinova ${ }^{1}$

${ }^{1}$ Institute of Biochemistry and Genetics - Subdivision of the Ufa Federal Research Centre of the Russian Academy of Sciences, Ufa, Russian Federation;

${ }^{2}$ Department of Medical Genetics and Fundamental Medicine, Bashkir State Medical University, Russian Federation;

${ }^{3}$ Psychological Institute of the Russian Academy of Education, Moscow, Russian Federation.

Keywords Depression, Oxytocin, Gene X Environment Interactions, Birth Order, Haplotype

Depression is an important public health issue due to its high prevalence and socio-economic costs to society. According to published findings, a multifactorial nature of depression development requires simultaneous examination of genetic and environmental factors. Considering the role of oxytocinergic functioning in emotional regulation, the present study aimed to estimate both the main effect of polymorphic variants and haplotypes of oxytocin receptor gene (OXTR: rs53576, rs237911, rs7632287, rs2254298, rs2228485, $r s 13316193$ ), and $\mathrm{G} \times \mathrm{E}$-interactions in individual differences in depression in healthy individuals considering modulating effect of environmental factors.

The study included 623 mentally healthy individuals $(81.11 \%$ women; $19.53 \pm 1.75$ years) of Caucasian origin (225 Russians, 218 Udmurts, 141 Tatars and 39 of mixed ethnicity) from Russia. Depression was assessed using BDI. SNPs genotyping was performed using PCR-based KASP genotyping technology on "CFX96" DNA Analyzer (BioRad, USA). Statistical analysis included multiple linear/ logistic regression followed by FDR-correction for multiple testing (PLINK v.1.09). Genotypes and 21 environmental parameters served as independent factors and depression level as dependent variable.

The present study revealed a modulating effect of birth order on association between $r s 237911(\beta=-2.742 ; \mathrm{P}=0.031)$ in the OXTR gene and depression level. $\mathrm{G} \times \mathrm{E}$ model including rs53576 ( $\beta=-$ 2.52; $\mathrm{P}=0.017)$ in the OXTR gene and the level of paternal care significantly modulated interindividual differences in depression. Haplotype analysis revealed an association of the OXTR GAG-haplotype (rs53576-rs2228485-rs237911) and enhanced depression in total sample $\left(\beta=4.967 ; \mathrm{P}_{\mathrm{FDR}}=0.034\right)$ and among women $(\beta=5.256$; $\mathrm{P}_{\mathrm{FDR}}=0.028$ ).

The present study indicated a modulating effect of environmental factors on $O X T R$-associated liability to depression in healthy individuals.

Grant Support: This study was supported by the Russian Foundation for Basic Research (project no. ofi-m-17-29-02195).

\section{Nature of nurture analysis via structural equation modeling}

Yongkang $\mathrm{Kim}^{1}$, Jared Balbona ${ }^{1,2}$, Matthew C Keller ${ }^{1,2}$

${ }^{1}$ Institute of Behavioral Genetics, University of Colorado at Boulder, Boulder, Colorado, USA

${ }^{2}$ Department of Psychology and Neuroscience, University of Colorado at Boulder, Boulder, Colorado, USA 
Keywords Heritability estimation, Nature of Nurture, Structural Equation Modeling (SEM), Genetics, OpenMX

While behavioral genetics is often viewed as being concerned with cataloging genetic variation and its determinants across traits, there has traditionally been great interest in understanding how family members directly impact each other in non-genetic ways. However, because family members share both genetic and environment factors, it is hard to disentangle variation due to genetic factors from variation due to familial shared environment factors by modeling only covariances between relatives.

Recently, Kong et al. developed a method to understand whether parental phenotypes directly influence their offspring phenotypes ("vertical transmission") using the covariance between a polygenic risk score from non-transmitted parental genotypes (which imperfectly reflects parental phenotype) and offspring phenotype. Here, we show how structural equation models (SEM) can both simplify and improve upon the method introduced by Kong et al. To estimate parameters of SEM model, we performed maximum likelihood (ML) estimation via openMX package in $\mathrm{R}$.

To verify our SEM model works similar to Kong et al. we used GeneEvolve to simulate several scenarios, including parent-to-offspring vertical transmission, assortative mating, and additive genetic effects. We show that our proposed method provides unbiased estimates of the full variance due to vertical transmission as well as estimates of genetic variation and (passive) gene-environment covariance. Path analysis provides an intuitive approach to modeling genetic nurture and allows the method to be expanded in a systematic and rigorous manner.

Grant Support: NIH R01 MH100141 (Keller).

\section{The association between body dysmorphic symptoms and suicidality among adolescents and young adults: a genetically-informative study}

Georgina Krebs ${ }^{1,2}$, Lorena Fernández de la $\mathrm{Cruz}^{3}$, Fruhling Rijsdijk ${ }^{1}$, Daniel Rautio $^{3}$, Jesper Enander ${ }^{3}$, Christian Rück ${ }^{3}$, Paul Lichtenstein ${ }^{4}$, Sebastian Lundström ${ }^{5}$, Henrik Larsson ${ }^{4,6}$, Thalia C Eley ${ }^{1, *}$, \& David Mataix-Cols ${ }^{3, *}$

*Joint last authors

${ }^{1}$ MRC Social, Genetic and Developmental Psychiatry Centre, Institute of Psychiatry, Psychology \& Neuroscience, King's College London, London, United Kingdom

${ }^{2}$ National and Specialist OCD and Related Disorders Clinic for Young People, South London and Maudsley NHS Foundation Trust, London, UK

${ }^{3}$ Centre for Psychiatry Research, Department of Clinical Neuroscience, Karolinska Institutet, \& Stockholm Health Care Services, Stockholm County Council, Stockholm, Sweden

${ }^{4}$ Department of Medical Epidemiology and Biostatistics, Karolinska Institutet, Stockholm, Sweden

${ }^{5}$ Gillberg Neuropsychiatry Centre, Centre for Ethics, Law and Mental Health, University of Gothenburg, Sweden

${ }^{6}$ School of Medical Sciences, Örebro University, Örebro, Sweden

Keywords BDD, Suicidal Ideation, Suicide Attempts, Twin Design, Genetic, Adolescence

Previous research indicates that body dysmorphic disorder (BDD) is associated with risk of suicidality. However, studies have relied on small and/or specialist samples and largely focused on adults, despite these difficulties commonly emerging in youth. Furthermore, the aetiology of the relationship remains unknown.
Two independent twin samples were identified through the Child and Adolescent Twin Study in Sweden, at ages $18(\mathrm{~N}=6027)$ and 24 ( $\mathrm{N}=3454)$. Participants completed a self-report measure of BDD symptom severity. Young people and parents completed items assessing suicidal ideation and suicide attempts. Logistic regression models tested the association between continuous scores of BDD symptoms and suicidality outcomes. Bivariate genetic models examined the aetiology of the association between BDD symptoms and a suicidality composite at both ages.

BDD symptoms were positively associated with suicidal ideation and suicide attempts at age 18 and 24 . These associations generally remained significant after adjusting for depressive and anxiety symptoms. Genetic factors accounted for most of the covariance between BDD symptoms and suicidality $(74.1 \%$ and $79.4 \%$ at ages 18 and 24 , respectively), but with significant non-shared environmental influences (25.9\% and $20.6 \%$ at ages 18 and 24, respectively).

BDD symptoms are associated with substantial risk of suicidal ideation and behaviours in late adolescence and early adulthood. This relationship is largely explained by common genetic liability, but non-shared environmental effects are also significant and could provide opportunities for prevention among those at high-risk.

Grant Support: The Swedish Twin Registry is managed by Karolinska Institutet and receives funding through the Swedish Research Council under the Grant No 2017-00641. The CATSS study is supported by Grants 2012-1678, 2014-0834 and 2017-02552 from the Swedish Council for Working Life, funds under ALF Agreement 2014-0322 and ALFGBG-776031, and Grants 340-2013-5867 and 2014-3831 from the Swedish Research Council. GK is funded by an MRC Clinical Research Training Fellowship (MR/N001400/1). TCE is part funded by a program grant from the MRC (MR/M021475/1).

\section{Cortical thickness and cortical surface area show differing genetic relationships with schizophrenia across development: a multiplex extended pedigree study}

Susan S. Kuo, M.S. ${ }^{1}$, David R. Roalf, Ph.D. ${ }^{3}$, Konasale Prasad, M.D. ${ }^{4}$, Christie W. Musket, M.S. ${ }^{1}$, Petra E. Rupert, B.S. ${ }^{1}$, Joel Wood $^{4}$, Ruben C. Gur, Ph.D. ${ }^{3}$, Laura Almasy, Ph.D. ${ }^{2}$, Raquel E. Gur, M.D., Ph.D. ${ }^{3}$, Vishwajit L. Nimgaonkar, M.D., Ph.D. ${ }^{4,5}$, Michael F. Pogue-Geile, Ph.D. ${ }^{1,4}$

${ }^{1}$ Department of Psychology, University of Pittsburgh

${ }^{2}$ Department of Genetics, University of Pennsylvania

${ }^{3}$ Department of Psychiatry, University of Pennsylvania

${ }^{4}$ Department of Psychiatry, University of Pittsburgh

${ }^{5}$ Department of Human Genetics, University of Pittsburgh

Keywords Schizophrenia, Imaging Genetics, Neurodevelopment, Brain Structure, Genetic Correlation

Schizophrenia genetic effects on neurodevelopment may remain stable from childhood (as proposed by early neurodevelopmental models; Murray and Lewis 1987; Weinberger 1987), increase during adulthood, closer to the peak age-of-onset (as proposed by late neurodevelopmental models; Feinberg 1983), or increase long after peak age-of-onset (as proposed by neurodegenerative models; Lieberman, 1999). To our knowledge, no study to date has directly tested schizophrenia developmental neurogenetic effects without confounding illness effects. To examine whether the genetic correlation between schizophrenia and brain morphology changes across peak age-of-onset, 200 relatives from 32 multiplex, extended pedigrees (age range: 12-85 years) and 276 unrelated controls underwent MRI scanning. Quantitative genetic variance decomposition analyses were 
conducted on MRI cortical thickness (CT) and cortical surface area (CSA) to parse schizophrenia developmental neurogenetic effects that emerge before schizophrenia peak age-of-risk, during peak age-ofrisk, and after peak age-of-risk. We found that genetic correlations between schizophrenia and cortical measures changed before, during, and after age-of-risk, suggesting late neurodevelopmental effects on temporal, fusiform, parahippocampal, cingulate, and insula CT and inferior temporal, fusiform, lingual, and pericalcarine CSA, in contrast to neurodegenerative effects on orbitofrontal, inferior frontal, and entorhinal CT. These developmental neurogenetic effects were notably specific to schizophrenia and not found in depression. Our findings suggest that the effects of schizophrenia genetic risk influence different aspects of brain structure before and after schizophrenia age-of-onset, emphasizing the importance of examining whether schizophrenia genetic risk variants change expression similarly across neurodevelopment.

\section{References}

Feinberg, I. (1983). Schizophrenia: caused by a fault in programmed synaptic elimination during adolescence? Journal of Psychiatric Research, 17(4), 319-334. doi:10.1016/0022-3956(82)90038-3.

Lieberman, J. A. (1999). Is schizophrenia a neurodegenerative disorder? A clinical and neurobiological perspective. Biological Psychiatry, 46(6), 729-739. doi:10.1016/S0006-3223(99)00147-X.

Murray, R. M., \& Lewis, S. W. (1987). Is schizophrenia a neurodevelopmental disorder? British Medical Journal, 295(6600), 681.

Weinberger, D. R. (1987). Implications of normal brain development for the pathogenesis of schizophrenia. Archives of General Psychiatry, 44(7), 660-669. doi:10.1001/archpsyc.1987.01800190080012

Grant Support: This research was supported by the Canadian Institutes of Health Research (SSK, Doctoral Foreign Study Award); National Institutes of Mental Health collaborative R01s, MH42191 (REG), MH63480 (VLN), and MH60722 (LA).

\section{Genetic fortune: winning or losing education, income, and health}

Hyeokmoon Kweon ${ }^{1}$, Casper A.P. Burik ${ }^{1}$, Richard Karlsson Linnér ${ }^{1}$, Ronald De Vlaming ${ }^{1}$, Aysu Okbay ${ }^{1}$, K. Paige Harden ${ }^{2}$, Thomas A. DiPrete $^{3}$, Philipp D. Koellinger ${ }^{1}$

${ }^{1}$ Department of Economics, Vrije Universiteit Amsterdam, Amsterdam, NL

${ }^{2}$ Department of Psychology, University of Texas at Austin, Austin, TX 78712, USA

${ }^{3}$ Department of Sociology, Columbia University, New York, NY 10027, USA

Keywords Income, Education, Health, Inequality, Polygenic Score

We examine the extent and the mechanisms through which genetic fortune contributes towards inequality in health and socioeconomic status. To this end, we conducted the first genome-wide association study (GWAS) of individual income, using data from individuals of European ancestries in the UK Biobank $(N=282,963)$. We augmented these GWAS results with GWAS estimates of educational attainment and constructed an aggregated genetic score. We exploit this score and random genetic differences between $\sim 35,000$ biological siblings to show that (i) roughly half of the covariance between our genetic score and socio-economic outcomes is causal, (ii) genetic luck for higher income is linked with better health outcomes in late adulthood, and (iii) having a college degree partly mediates this relationship. Thus, genetic factors contribute towards inequality, but these effects are malleable, for example via policies targeting education.

Grant Support: This study was supported by funding from a European Research Council Consolidator Grant (647648 EdGe) to Koellinger and by a Jacobs Foundation Grant to Harden.

\section{Examining the causal link between divorce and mental health of their offspring using the cross-generational sibling comparison model}

Kennon Later ${ }^{1}$, Jonathan Trattner $^{2}$, Carlos Santos ${ }^{1}$, S. Mason Garrison $^{1}$

${ }^{1}$ Department of Psychology, Wake Forest University Winston-Salem, North Carolina, USA

${ }^{2}$ Department of Interdisciplinary Studies, Wake Forest University Winston-Salem, North Carolina, USA

Keywords Sibling-comparison; Divorce; Mental health; Causal Inference; Cross-Generation

Children of divorce have increased depression, anxiety, and perform worse in school. These consequences affect subsequent generations (Amato and Cheadle 2005), suggesting a heritable 'divorce-induced' epigenetic modification or a genetic mediation as a result of neuroticism and extraversion (Jocklin et al. 1996). Therefore, we aim to understand whether divorce actually affects an individual's wellbeing or reflects those same genetic components.

Using the National Longitudinal Survey of Youth 1979, we examined the cross-generational impact of divorce on mental health (according to the Behavior Problems and Child Depression Report), following individuals of different ages. We compared maternal cousins using the discordant kinship model (Garrison and Rogers 2016). Controlling for genetic confounds, results suggest that divorce does not impact children's mental health by itself, however, the timing of a divorce might be a factor.

\section{References}

Amato, P. and Cheadle, J. (2005), The long reach of divorce: Divorce and child well-being across three generations. Journal of Marriage and Family, 67: 191-206. doi:10.1111/j.0022-2445.2005.00014.x. Garrison, S. M., \& Rodgers, J. L. (2016). Casting doubt on the causal link between intelligence and age at first intercourse: A cross-generational sibling comparison design using the NLSY. Intelligence, 59, 139-156.

Jocklin, V., McGue, M., \& Lykken, D. T. (1996). Personality and divorce: A genetic analysis. Journal of Personality and Social Psychology, 71(2), 288-299. https://doi.org/10.1037/0022-3514.71.2.288

\section{Genetic and environmental aetiologies of suicidal and non-suicidal self-harm}

Kai Xiang Lim $^{1}$, Kaili Rimfeld ${ }^{1}$, Jean-Baptiste Pingault ${ }^{1,2}$, Frühling Rijsdijk ${ }^{1}$.

${ }^{1}$ Social, Genetic and Developmental Psychiatry Centre, Institute of Psychiatry, Psychology \& Neuroscience, King's College London, London, United Kingdom

${ }^{2}$ Division of Psychology and Language Sciences, University College London, London, United Kingdom 
Keywords Self-Harm; Mental Health; Suicidal Self-Harm; Non-Suicidal Self-Harm

Self-harm can be further refined into suicidal self-harm (SSH) and non-suicidal self-harm (NSSH). Using a twin design, we aimed to: (1) investigate to what extent SSH and NSSH share the same or distinct genetic and environmental aetiologies; and (2) investigate the shared genetic and environmental overlap between a range of psychiatric symptoms with both SSH and NSSH.

We analysed data from the Twins Early Development Study (TEDS). A total of 9085 twins (62.4\% female) answered questions related to lifetime presence of self-harm at age 21. Parent-reported and self-reported mental health measures were collected at age 16. Genetic SEM analyses were performed using raw maximum likelihood estimation in OpenMx (Boker et al. 2011).

Heritability estimates for NSSH $(0.54,95 \% \mathrm{CI}: 0.35-0.60)$ and SSH $(0.49,95 \%$ CI: $0.22-0.58)$ are similar. There were no effects of shared environmental influences. Additive genetic factors explain $56.3 \%$ of the phenotypic correlation $(r=0.87)$ between NSSH and $\mathrm{SSH}$. The genetic and environmental correlations between NSSH or SSH with the mental health measures show no statistically significant difference.

This study shows that there is genetic influence on NSSH and $\mathrm{SSH}$, with over half of their correlation explained by shared genetic factors. No distinct pattern of associations between mental health measures and either NSSH or SSH was observed. Together, the evidence suggests no aetiological difference between NSSH and SSH.

References

S. Boker et al., "OpenMx: An Open Source Extended Structural Equation Modeling Framework," Psychometrika, vol. 76, no. 2, pp. 306-317, Apr. 2011.

Grant Support: King's International Postgraduate Research Scholarship

\section{Exploring the genetic architecture of disordered gambling and the influence of genetics risk of gambling on substance use}

Penelope A Lind ${ }^{1}$, Ian R Gizer ${ }^{2}$, Thomas M Piasecki², Wendy S Slutske $^{2}$, Nicholas G Martin ${ }^{3}$

${ }^{1}$ Psychiatric Genetics Group, QIMR Berghofer Medical Research Institute, Brisbane, QLD, Australia

${ }^{2}$ Department of Psychological Sciences, University of Missouri, Columbia, MO, USA

${ }^{3}$ Genetic Epidemiology Group, QIMR Berghofer Medical Research Institute, Brisbane, QLD, Australia

Keywords Disordered Gambling, GWAS, Polygenic Risk Scores, Substance Use

Disordered gambling (DG) refers to the full continuum of problems related to excessive gambling that include pathological gambling symptomatology and versatility of gambling involvement. DG also has a high level of comorbidity with substance use. The underlying genetic basis is largely unknown; few candidate gene studies and only two genome-wide association studies (GWAS) of gambling-related traits have been published. Using self-report data from the Australian Twin Study of Gambling and Australian Genetics of Depression Study, we performed the largest GWAS to date $\left(\mathrm{n}_{\max }=14,646\right)$, for three measures of gambling behaviour: ever gambled (EVRGAM), versatility of gambling involvement (VERS), and a DG factor score (DGF). We identified two independent genome-wide significant loci for VERS and one for DGF $(P<5 \mathrm{E}-8)$. GCTA SNP-based heritability estimates were $1.7 \%$ (EVRGAM), 13.4\% (VERS) and $11.2 \%$ (DGF). Next, we calculated polygenic risk scores (PRS) from the GWAS summary for a range of p-value thresholds. Using LMM, we then examined the extent to which the PRS could predict gambling behaviour and substance use (alcohol and tobacco use, and total number of substances ever used [subVERS]) in the Australian 25UP Study $\left(\mathrm{n}_{\max }=2155\right)$. PRS $\mathrm{PERS}_{\text {Vas }}$ most predictive of gambling behaviours (explaining $0.8 \%$ and $0.45 \%$ of variance in VERS in DGF) and frequency of alcohol use $\left(\mathrm{p}=0.0071, \mathrm{R}^{2}=0.40 \%\right)$. The best predictive value of the $\mathrm{PRS}_{\mathrm{DGF}}$ was observed for cigarettes per day ( $\mathrm{p}$ $\left.=0.0079, \mathrm{R}^{2}=1.65 \%\right)$ and subVERS $\left(\mathrm{p}=0.0098, \mathrm{R}^{2}=0.37 \%\right)$.

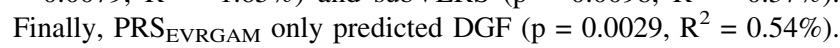

\section{Genetic, lifestyle and environmental risk factors for chronic pain revealed through GWAS}

Mischa Lundberg ${ }^{1,2}$, Adrian I. Campos ${ }^{3,4}$, Scott F. Farrel1 ${ }^{5,6,7}$, Geng Wang $^{1}$, Michele M. Sterling ${ }^{5,6}$, Miguel E. Rentería ${ }^{3,4}$, Trung Thanh Ngo $^{1}$, Gabriel Cuéllar-Partida ${ }^{1}$

${ }^{1}$ The University of Queensland Diamantina Institute, The University of Queensland, Woolloongabba, Australia

${ }^{2}$ Transformational Bioinformatics, Commonwealth Scientific and Industrial Research Organisation, Sydney, New South Wales, Australia

${ }^{3}$ Genetic Epidemiology Lab, QIMR Berghofer Medical Research Institute, Herston, Australia

${ }^{4}$ Faculty of Medicine, The University of Queensland, Herston, Australia

${ }^{5}$ RECOVER Injury Research Centre, The University of Queensland, Brisbane, Australia

${ }^{6}$ NHMRC Centre of Research Excellence in Road Traffic Injury Recovery, The University of Queensland, Brisbane, Australia

${ }^{7}$ Menzies Health Institute Queensland, Griffith University, Gold Coast, Australia

Keywords Genome-Wide Association Study (GWAS), Persistent Pain, Risk Factors, Genetic Causality Proportion, Mendelian Randomization

Chronic pain is a leading cause of disability worldwide with complex aetiologies that remain elusive. Here we explore the genetic architecture of chronic pain by performing a genome-wide association study on 188,352 cases and 69,627 controls from the UK Biobank. We identified two independent genome-wide significant loci $(P<$ $5 \times 10^{-8}$ ) associated with chronic pain near ADAMTS6 (rs113313884) and LEMD2 (rs10660361). Using gene-based association tests, we identified genetic variants associated with chronic pain (associated genes: DCAKD, NMT1, MLN, IP6K3; $P<2 \times 10^{-6}$ ). Furthermore, genetic correlation $\left(\mathrm{r}_{\mathrm{G}}\right)$ analyses revealed largely similar genetic influences between male and female patients $\left(\mathrm{r}_{\mathrm{G}}=1\right)$, suggesting individual differences in the presentation of chronic pain may emerge due to environmental exposures and lifestyle factors. Using linkage disequilibrium score-regression applied to 1328 complex traits, 548 (41\%) were found to be genetically correlated with chronic pain (FDR $<5 \%)$, of which 175 (13\%) showed genetic causal relationships using the latent causal variable model and Generalised Summarydata-based Mendelian Randomisation. In particular, major depressive disorder, anxiety, smoking, body fat, BMI and musculoskeletal diseases were found to increase the risk of chronic pain, whereas diet, walking for pleasure and higher educational attainment were associated with a reduced risk. In conclusion, this data-driven hypothesisfree approach has uncovered several specific risk factors that warrant 
further examination to help deliver effective early screening and management strategies for chronic pain.

Grant Support: University of Queensland Research Training Stipend; University of Queensland Research Training Tuition Fee Offset; CSIRO PhD Top Up Scholarship

\section{Sleep quality and psychological distress: a sex- limitation twin study}

Madrid-Valero, J.J ${ }^{1}$., Kirkpatrick, R.M ${ }^{2}$., González-Javier, $\mathrm{F}^{3}$., Gregory, A.M. ${ }^{4}$ and Ordoñana, J.R ${ }^{3}$

${ }^{1}$ Department of Health Psychology, University of Alicante, Alicante, Spain.

${ }^{2}$ Virginia Institute for Psychiatric \& Behavioral Genetics, Virginia Commonwealth University, Richmond, Virginia, USA

${ }^{3}$ Department of Human Anatomy and Psychobiology, University of Murcia, Murcia, Spain

${ }^{4}$ Department of Psychology, Goldsmiths, University of London, London, UK

Keywords Depression, Anxiety, Sex, Sleep quality.

Further research is needed to understand why anxiety/depression symptoms so commonly co-occur with poor sleep quality. As compared to men, women usually report more sleep problems, such as insomnia and poorer sleep quality (Wang et al. 2020; Zhang and Wing 2006). A similar picture occurs for anxiety and mood disorders, where women usually have a higher prevalence of these disorders as compared to men (Albert 2015; McLean et al. 2011). Further research is needed to investigate the genetic and environmental influences on this relationship taking in to account possible sex differences. The current sample comprised 2150 participants from the Murcia Twin Registry. The sample was $55.7 \%$ female and the mean age was 53.7 $(\mathrm{SD}=7.4)$. Sleep quality was measured using the Pittsburgh Sleep Quality Index and psychological distress using the "anxiety/depression" dimension of the EuroQol-5D questionnaire. A joint (means/ threshold) sex-limitation model was fitted to test for possible sex differences. The best fitting model was provided by a non-scalar AE model where estimates for psychological distress were similar for males $(\mathrm{A}=51 \% ; 95 \%$ CI $33-75 \% ; \mathrm{E}=49 \% ; 95 \%$ CI $24-67 \%)$ and females $(\mathrm{A}=50 \%$; $95 \%$ CI 34-69\%; $\mathrm{E}=50 \%$; 95\% CI 31-66\%). However, different estimates were found for males $26 \%(95 \% \mathrm{CI}$ $8-43 \%$ ) and females $40 \%$ (95\% CI 28-50\%) regarding sleep quality. A significant genetic correlation between sleep quality and psychological distress was also found, but only for females ( $\mathrm{rA}=0.59 ; 95 \%$ CI $0.36,0.84)$. Our results confirm the strong genetic association between sleep quality and psychological distress. Although, our results point to a differential association between the sexes, future studies with greater statistical power are required to confirm these findings.

\section{References}

Albert, P. R. (2015). Why is depression more prevalent in women? $J$ Psychiatry Neurosci, 40(4), 219-221.

McLean, C. P., Asnaani, A., Litz, B. T., \& Hofmann, S. G. (2011). Gender differences in anxiety disorders: prevalence, course of illness, comorbidity and burden of illness. J Psychiatr Res, 45(8), 1027-1035. doi:10.1016/j.jpsychires.2011.03.006

Wang, P., Song, L., Wang, K., Han, X., Cong, L., Wang, Y., . . D Du, Y. (2020). Prevalence and associated factors of poor sleep quality among Chinese older adults living in a rural area: a population-based study. Aging Clin Exp Res, 32(1), 125-131. doi:10.1007/s40520-01901171-0
Zhang, B., \& Wing, Y. K. (2006). Sex differences in insomnia: a meta-analysis. Sleep, 29(1), 85-93.

Grant Support: Ministerio de Ciencia, Innovación y Universidades Spain (RTI2018-095185-B-I00) co-funded by European Regional Development Fund (FEDER)

\section{Sleep quality and back pain: same genes?}

Madrid-Valero, J.J ${ }^{1}$., Andreucci, $\mathrm{A}^{2}$., Carrillo-Verdejo, $\mathrm{E}^{3}$., Ferreira, $\mathrm{PH}^{4}$, Martínez-Selva, J.M ${ }^{3}$ and Ordoñana, J.R ${ }^{3}$.

${ }^{1}$ Department of Health Psychology, University of Alicante, Alicante, Spain.

2 Department of Clinical Medicine, Aalborg University, Aalborg, Denmark

${ }^{3}$ Department of Human Anatomy and Psychobiology, University of Murcia, Murcia, Spain

${ }^{4}$ Discipline of Physiotherapy, Faculty of Health Sciences, The University of Sydney, Sydney, Australia

Keywords Low back pain, Neck Pain, Sleep quality.

Sleep quality (SQ), chronic Low Back Pain (LBP) and chronic Neck Pain (NP) are genetically influenced; all three conditions frequently co-occur; and shared genetic etiology on a pairwise base has been reported. However, to our knowledge no study has investigated if these three conditions are influenced by the same genetic and environmental factors and the extent and pattern of genetic overlap between them yet. The sample comprised 2134 participants of the Murcia Twin Registry with a mean age of 53.77(SD = 7.2). The sample was $54.6 \%$ female. SQ was measured by means of the Pittsburgh Sleep Quality Index Questionnaire. Lifetime prevalence of both NP and LBP were assessed through a dichotomous self-reported question derived from the Spanish National Health Survey. A multivariate model and a common pathway model with two latent factors were fitted in order to estimate the proportion of the variance accounted for: (1) specific genetic and environmental factors on LBP and NP separately; (2) genetic and environmental factors on a latent factor for back pain (encompassing LBP and NP); and (3) common genetic and environmental factors shared by SQ and the latent back pain factor. Variance of the latent back pain factor is explained by both genetic $(41 \%)$ and environmental $(59 \%)$ factors. These results also showed that genes affecting SQ only contribute with a $3 \%$ of the variance to the common latent back pain variable and only $5 \%$ of the variance on the latent pain factor is shared with environmental factors related to $S Q$.

Grant Support: Funding: Ministerio de Ciencia, Innovación y Universidades - Spain (RTI2018-095185-B-I00) co-funded by European Regional Development Fund (FEDER)

\section{Pathfinder: a brief, reliable measure to overcome the challenges of investigating $g$ in the genomic era}

Margherita Malanchini ${ }^{1,2,3, *}$ Kaili Rimfeld ${ }^{2, *}$, Agnieszka Bubel ${ }^{2}$, Rosa Cheesman ${ }^{2}$, Andrea G. Allegrini ${ }^{2}$, Nicholas G. Shakeshaft ${ }^{2,4}$, Kerry Schofield ${ }^{2,4}$, Amy Packer ${ }^{2}$, Rachel Ogden ${ }^{2}$, Andrew McMil$\operatorname{lan}^{2}$, Sophie von Stumm ${ }^{5}$ and Robert Plomin ${ }^{2}$

${ }^{1}$ School of Biological and Chemical Sciences, Queen Mary University of London

${ }^{2}$ Social Genetic \& Developmental Psychiatry Centre, Institute of Psychiatry, Psychology \& Neuroscience, King's College London, UK ${ }^{3}$ Population Research Center, The University of Texas at Austin 
${ }^{4}$ QuodIt Ltd.

${ }^{5}$ Department of Education, University of York

*Joint first authors

Genome-wide association (GWA) studies using diverse measures of general cognitive ability $(g)$ have produced polygenic scores that significantly predict $g$, although their predictive power is far from capturing the heritability estimates of twin studies. One strategy to bridge this missing heritability gap is to incorporate the same highquality measure of $g$ in large biobanks.

We created a 15-minute online measure of $g$ that is reliable and psychometrically valid. The final $g$ measure includes 20 items measuring verbal ability and 20 measuring nonverbal ability, embedded in a gamified storyline: Pathfinder. We administered this measure to 4200 young adults from the Twins Early Development Study.

Our measure of $g$ correlated strongly $(r=.83)$ with a large battery of well-established verbal and nonverbal tests and showed excellent test-retest reliability $(r=.81)$. Twin analyses showed that these new measures of $g$, verbal ability and nonverbal ability were substantially heritable $(57 \%, 62 \%$ and $45 \%$, respectively). A polygenic score derived from the latest GWA study of intelligence accounted for $9.3 \%$ of the variation in $g$; a polygenic score computed from GWA studies of five cognitive and educational traits accounted for $12.3 \%$ of the variation. This new $g$ measure correlated substantially with other well-established measures of $g$ collected in the same sample over development (average $r=.50$ ), academic achievement (average $r=$ .48 ) and socio-economic status (average $r=.30$ ).

It is our hope that this brief, reliable and fun measure of $g$ will be used widely to advance research in genomics and the social and behavioural sciences.

\section{Effects of genetic likelihood for extraversion/surgency on preschoolers' prosocial behaviors in ambiguous situations}

\author{
Riley L. Marshall ${ }^{1}$ \& Lisabeth Fisher DiLalla ${ }^{1,2}$ \\ ${ }^{1}$ Department of Psychology, Southern Illinois University, Carbondale, \\ IL, USA \\ ${ }^{2}$ School of Medicine, Southern Illinois University, Carbondale, IL, \\ USA
}

Keywords Prosocial, Ambiguous, Extraversion/Surgency, Agreeableness, Twins

The ability to behave prosocially in ambiguous situations is an important skill for navigating diverse interpersonal situations. The development of prosocial behavior may be influenced by parenting, particularly in shy children; less is known about extraverted children. We examined the interaction between genetic likelihood for temperamental extraversion/surgency and parent agreeableness on children's prosocial behaviors in ambiguous situations. We hypothesized that parent agreeableness would be related to children's prosociality differentially depending on children's extraversion/surgency.

Children from the Southern Illinois Twins/Triplets and Siblings Study (DiLalla \& Jamnik 2019; 72 MZ, 108 DZ) were tested at age 5 on prosocial responses using a picture vignette series. Parents rated child temperament at age 4 and their own personality when children were 5. A genetic likelihood index was calculated for extraversion/surgency (GLIE) based on co-twin's temperament and genetic relatedness.

Multilevel modeling showed a significant interaction between GLIE and maternal agreeableness predicting child prosocial responses in ambiguous social situations. Children at genetic risk for low scores on extraversion/surgency were significantly more likely to give prosocial responses if their mothers were more agreeable. Maternal agreeableness had no effect for children genetically likely to be high on extraversion/surgency.

Thus, children at genetic risk for low extraversion/surgency were at risk of responding less prosocially unless their mothers are highly agreeable. Interventions aimed at increasing prosociality in shy children should focus on increasing positivity in their rearing environments.

\section{References}

DiLalla, L. F. \& Jamnik, M. R. (2019). The Southern Illinois Twins/Triplets and Siblings Study (SITSS): A longitudinal study of early child development. Twin Research and Human Genetics.

\section{Sex differences in psychiatric polygenic risk in children with ADHD}

Joanna Martin ${ }^{1}$, Sharifah Shameem Agha ${ }^{1,2}$, Olga Eyre ${ }^{1}$, Lucy Riglin $^{1}$, Leon Hubbard ${ }^{1}$, Kate Langley ${ }^{3}$, Ben Neale ${ }^{4}$, Anita Thapar ${ }^{1}$, Michael O'Donovan ${ }^{1}$

${ }^{1}$ MRC Centre for Neuropsychiatric Genetics and Genomics, Division of Psychological Medicine and Clinical Neurosciences, Cardiff University, Cardiff, UK

${ }^{2}$ Cwm Taf Morgannwg University Health Board Health Board, Wales, UK

${ }^{3}$ School of Psychology, Cardiff University, Cardiff, UK

${ }^{4}$ Stanley Center for Psychiatric Research, Broad Institute, Cambridge, MA, USA

Keywords ADHD; anxiety disorder; depression; sex differences; polygenic risk scores

Attention deficit hyperactivity disorder (ADHD) is more commonly diagnosed in males than females. It is frequently comorbid with and shares genetic risks with anxiety and depression, which are more common in females in the general population. We tested for sex differences in polygenic risk scores (PRS) for anxiety and depression in children with ADHD and examined possible sex differences in the association between anxiety and depression PRS with anxiety and depression symptoms in the context of ADHD. Exploratory analyses tested for sex differences in PRS for other psychiatric disorders.

Children with ADHD were recruited from clinics and symptoms of anxiety (separation, social and generalised) and depression were assessed using a standardised diagnostic interview. We derived PRS based on large genetic studies of anxiety, major depressive disorders, ADHD, autism, bipolar disorder, and schizophrenia.

In 885 children with ADHD (14\% female), we found no sex differences in comorbid anxiety and depression symptoms or anxiety/ depression PRS. Exploratory analyses suggested higher PRS for bipolar disorder in females [OR(CIs) $=1.23(1.00-1.51)]$ and this was replicated using an independent ADHD sample $[\mathrm{OR}(\mathrm{CIs})=1.0$ 9(1.00-1.19)]. Anxiety PRS were associated with social [OR(CIs) = 1.54 (1.14-2.09)] and generalised anxiety symptoms [OR(CIs) $=1.46$ (1.11-1.92)] in males but not females, with significant sex-by-PRS interactions. Depression PRS were not associated with depression symptoms.

The results support the presence of sex differences in children with ADHD, indicating that the genetic link between ADHD and bipolar disorder may be stronger in females and that the aetiology of comorbid anxiety problems may differ in males and females with ADHD.

Grant Support: The work was supported by a Sêr Cymru II COFUND Fellowship from the Welsh Government (Grant No. 663830 - 
CU189), as well as funding from the Wellcome Trust (grant no. 079711), Medical Research Council Centre (Grant No. MR/L010305/ 1), Health and Care Research Wales (Grant No. 514032), Action Medical Research and Baily Thomas.

\section{Grip strength and lung function associations: a middle childhood twin study}

Samantha A. Miadich, Mary C. Davis, Leah D. Doane, \& Kathryn Lemery-Chalfant

Department of Psychology, Arizona State University, Tempe, Arizona, USA

Keywords Lung Function, Grip Strength, Twins, Children

While grip strength and lung function assessments have been related to each other later in life, much is unknown about their associations in childhood. This study examined: ((1) genetic, shared environmental, and non-shared environmental influences on lung function (i.e., FEV1, PEF) and grip strength, and (2) whether the covariance between grip strength and lung function is due to genetic and/or environmental influences.

A subset of 333 families (663 twins; $31 \%$ MZ, 35\% same-sex DZ, $35 \%$ opposite-sex DZ twins) from the Arizona Twin Project (LemeryChalfant et al. 2019, Twin Research \& Human Genetics, 22, 681-685) were included in this study. At approximately 9 years $(M=9.70, S D=$ 0.88 ), twins $(50 \%$ female; $58.8 \%$ non-Hispanic White, $29.3 \%$ Hispanic) completed physical health assessments during a home visit including grip strength, lung function, and height/weight measurements.

Univariate ACE models indicated strong genetic influences on grip strength (78\%) and FEV1 (74\%) and moderate genetic influences on PEF (58\%). Bivariate Cholesky decompositions revealed that the covariance between grip strength and both FEV1 (full model: $r \mathrm{~A}=$ $0.29, r \mathrm{D}=0.49, r \mathrm{E}=0.00$ ) and PEF (full model: $r \mathrm{~A}=0.39, r \mathrm{D}=0.05$, $r \mathrm{E}=0.19)$ was genetic. Additive genetic and non-shared environmental influences explained the covariance between FEV1 and PEF (full model: $r \mathrm{~A}=0.77, r \mathrm{C}=0.00, r \mathrm{E}=0.58$ ). Models accounted for child sex, age, height, and waist circumference.

Findings from this study suggest a genetic commonality between grip strength and lung function and the need to focus on shared and unique risk factors when promoting muscular strength and pulmonary health in childhood with implications for overall health (e.g., cardiorespiratory fitness).

Grant Support: R01 HD079520 Social and Genetic Contributions to Children's Sleep, Health, and Functioning; R01 HD086085 Genetic and Environmental Origins of the Development of Pain in Children

\section{Patterns of item nonresponse to questionnaires are systematic and have a genetic basis}

Gianmarco Mignogna, Caitlin Carey ${ }^{1}$, Nikolas Baya ${ }^{1}$, Mattia Cordioli $^{2}$, Nicola Pirastu ${ }^{3}$, Rino Bellocco ${ }^{4}$, Peter Joshi ${ }^{5}$, John R.B. Perry ${ }^{6}$, Michel G. Nivard ${ }^{7}$, Benjamin M. Neale ${ }^{1}$, Raymond Walters ${ }^{1}$, Andrea Ganna $^{2}$

${ }^{1}$ Analytic and Translational Genetics Unit, Massachusetts General Hospital, Boston, MA, USA

${ }^{2}$ Institute for Molecular Medicine Finland, University of Helsinki, Helsinki, Finland

${ }^{3}$ Centre for Global Health Research, Usher Institute, University of Edinburgh, Edinburgh
${ }^{4}$ Department of Statistics and Quantitative Methods, University of Milano Bicocca, Milan, Italy

${ }^{5}$ UsherInstitute,UniversityofEdinburgh,Edinburgh

${ }^{6}$ MRC Epidemiology Unit, School of Clinical Medicine, University of Cambridge, Cambridge, UK

${ }^{7}$ Faculty of Behavioural and Movement Sciences, Biological Psychology, Amsterdam, the Netherlands

Keywords Item-Non-Response, No Answer, Non Response

Item nonresponse occurs when no substantive answer is recorded for a study participant on a given item. This nonresponse is interesting both as a behavioural choice and as a statistical concern from the resulting missing data. The current study aims to evaluate the contribution of genetics in item nonresponse between individuals. We examined this behaviour in 109 items from the initial survey of UK Biobank ( $\mathrm{N}=$ 360,628 ), focusing on the options "Prefer not to answer" (PNA) and "I don't know" (IDK).

GWAS of the two nonresponding behaviours identified 39 genome-wide significant loci and significant SNP-heritability for both PNA $\left(h^{2}=2.04 \%, \mathrm{P}=3 \times 10^{-16}\right)$ and $\operatorname{IDK}\left(\mathrm{h}^{2}=6.75 \%, \mathrm{P}=4 \times\right.$ $\left.10^{-112}\right)$. PNA and IDK are highly genetically correlated with each other $(\mathrm{rg}=0.73,[0.70,0.76])$ and with socioeconomic factors. Genomic SEM allowed to 'subtract' the genetic effect of IDK from PNA and vice-versa. IDK-adjusted PNA maintained a significant association with lower EA and income $(\mathrm{rg}=-0.37[-0.31,-0.43]$, for both) and poorer general health $(\mathrm{rg}=0.26[0.21,0.31])$. IDKadjusted PNA became associated with bipolarism $(\mathrm{rg}=0.25[0.13$, $0.37])$ and schizophrenia $(\mathrm{rg}=0.30[0.23,0.37])$. The genetic variance unique of PNA and IDK after subtracting the SES effect was $1.33 \%\left(\mathrm{P}=2 \times 10^{-16}\right)$ and $5.09 \%\left(\mathrm{P}=9 \times 10^{-78}\right)$, respectively.

Both PNA and IDK capture a similar socioeconomic background among nonresponders; although, while IDK reflects a more random nonresponding behaviour, PNA-specific genetic signal was positively associated with psychiatric disorders.

\section{Context matters: socioeconomic disadvantage increases phenotypic risk and impacts the etiology of disordered eating in youth}

Megan E. Mikhail ${ }^{1}$, Sarah L. Carroll ${ }^{1}$, D. Angus Clark $^{2}$, Shannon $\mathrm{O}^{\prime}$ Connor $^{3}$, S. Alexandra Burt ${ }^{1}$, \& Kelly L. Klump ${ }^{1}$

${ }^{1}$ Department of Psychology, Michigan State University, East Lansing, Michigan, USA

${ }^{2}$ Department of Psychiatry and Addiction Center, University of Michigan, Ann Arbor, Michigan, USA

${ }^{3}$ Department of Psychiatry and Behavioral Neuroscience, University of Chicago, Chicago, Illinois, USA

Keywords Disordered Eating, Etiology, Youth, Gene X Environment Interaction

Emerging evidence suggests food insecurity and other correlates of low socioeconomic status (SES) may increase disordered eating; however, few population-based studies have been conducted, and none have looked at the impact of SES on etiology. We hypothesized that stressors associated with socioeconomic disadvantage may potentiate latent genetic risk in vulnerable individuals and lead to more disordered eating (i.e., a diathesis-stress model), as has been observed for related phenotypes (e.g., depression). To investigate this possibility, we examined family SES as a potential moderator of the etiology of disordered eating in 10,788 male and female twins ages 8-18 from the Michigan State University Twin Registry. Parents rated the twins on nine items assessing core disordered eating symptoms (e.g., weight preoccupation, binge eating). Family SES was computed 
as a latent variable factor score from family income and mother and father's education level. Preliminary analyses at the phenotypic level indicated that lower family SES was associated with more disordered eating symptoms in both boys and girls even after controlling for potential confounds (e.g., race, pubertal status). $\mathrm{G} \times \mathrm{E}$ twin models controlling for age, BMI percentile, and pubertal status suggested that lower family SES was associated with increased genetic influences on disordered eating in girls and increased environmental influences on disordered eating in boys. Contrary to stereotypes of eating disorders as illnesses of privilege, this study is the first to demonstrate the etiologic pathways through which socioeconomic disadvantage may potentiate risk for disordered eating in youth.

Grant Support: This work was supported by a grant from the National Institute of Mental Health (R01 MH111715). The content of the manuscript is solely the responsibility of the authors and does not necessarily represent the official views of the National Institute of Mental Health.

\section{Exploring the genetic overlap underlying Alzheimer's disease and hearing loss}

Brittany L. Mitchell ${ }^{1,2}$, Jackson G. Thorp ${ }^{1,3}$, David M. Evans ${ }^{4,5}$, Dale R. Nyholt ${ }^{2}$, Nicholas G. Martin ${ }^{1,2}$ and Michelle K. Lupton ${ }^{1,2}$.

${ }^{1}$ Department of Genetics and Computational Biology, QIMR Berghofer Medical Research Institute, Australia.

${ }^{2}$ School of Biomedical Science and Institute of Health and Biomedical Innovation, Faculty of Health, Queensland University of Technology, Australia.

${ }^{3}$ Faculty of Medicine, University of Queensland, Australia.

${ }^{4}$ The University of Queensland Diamantina Institute, The University of Queensland, Brisbane, Austrlia

${ }^{5}$ MRC Integrative Epidemiology Unit, University of Bristol, Bristol, United Kingdom.

Keywords Alzheimer's disease, hearing, genetic aetiology

Alzheimer's disease (AD) is a neurodegenerative disease that is characterized by a progressive decline in neuronal function that leads to cognitive, behavioural and motor dysfunction over time. It is well established that several non-modifiable risk factors for AD, such as age and genetics, exist. However, as these cannot be targeted to reduce disease progression, emphasis on identifying modifiable risk factors is paramount. Hearing loss has recently been identified as the potentially largest modifiable risk factor for $\mathrm{AD}$ (OR 1.94), estimated to account for a similar increase in AD risk to APOE $\varepsilon 4$ (Livingston et al. 2017). However, the mechanisms underlying this association are still poorly understood. In this study, we investigate whether the relationship between hearing loss and $\mathrm{AD}$ is due to shared genetic aetiology, and seek evidence for a causal relationship between the traits. We found a significant genetic correlation between hearing difficulty, the use of a hearing aid and $\mathrm{AD}$ and polygenic risk score for $\mathrm{AD}$ was able to significantly predict hearing loss in an independent cohort. Additionally, regions of the genome involved in inflammation and immune response were identified to be shared between hearing difficulty and AD using Pairwise-GWAS. However, causality tests found no significant evidence of a causal relationship between these traits in either direction. Overall, these results currently do not support the hypothesis that hearing difficulty is a modifiable risk factor for $\mathrm{AD}$ and that the simple management of hearing loss will likely not mitigate $\mathrm{AD}$ risk.

References
Livingston $\mathrm{G}$ et al. Dementia prevention, intervention, and care. The Lancet. 2017;390(10113):2673-734.

\section{Do maternal intrauterine environmental influences that lower offspring birthweight causally increase offspring cardiometabolic risk factors in later life? A Mendelian randomization study of 45,849 genotyped parent offspring pairs in the HUNT study using the OpenMx software}

Gunn-Helen Moen ${ }^{1,2,3,4}$, Ben Brumpton ${ }^{3,5,6}$, Cristen Willer ${ }^{7,8,9}$, Bjørn Olav Åsvold ${ }^{3,10}$, Kåre Birkeland ${ }^{1}$, Michael C Neale ${ }^{11}$, Rachel M Freathy ${ }^{12}$, George Davey Smith ${ }^{4,6,13}$, Deborah A Lawlor ${ }^{4,6,13}$, Robert M Kirkpatrick ${ }^{11}$, Nicole M Warrington ${ }^{2,3,6^{*}}$, David M Evans ${ }^{2,6^{*}}$

${ }^{1}$ Institute of Clinical Medicine, Faculty of Medicine, University of Oslo, Norway.

${ }^{2}$ The University of Queensland Diamantina Institute, The University of Queensland, Woolloongabba, QLD 4102, Australia.

${ }^{3}$ K.G. Jebsen Center for Genetic Epidemiology, Department of Public Health and Nursing, NTNU, Norwegian University of Science and Technology, Norway.

${ }^{4}$ Population Health Science, Bristol Medical School, University of Bristol, UK.

${ }^{5}$ Department of Thoracic and Occupational Medicine, St. Olavs Hospital, Trondheim University Hospital, Trondheim, Norway

${ }^{6}$ Medical Research Council Integrative Epidemiology Unit at the University of Bristol, Bristol, UK

${ }^{7}$ Department of Biostatistics and Center for Statistical Genetics, University of Michigan, Ann Arbor, USA

${ }^{8}$ Department of Internal Medicine, University of Michigan, Ann Arbor, MI, USA

${ }^{9}$ Department of Human Genetics, University of Michigan, Ann Arbor, USA

${ }^{10}$ Department of Endocrinology, St. Olavs Hospital, Trondheim University Hospital, Trondheim, Norway

${ }^{11}$ Department of Psychiatry, Virginia Institute for Psychiatric and Behavioral Genetics, Virginia Commonwealth University, Richmond, VA, USA

${ }^{12}$ Institute of Biomedical and Clinical Science, College of Medicine and Health, University of Exeter, Exeter, UK

${ }^{13}$ Bristol NIHR Biomedical Research Centre, Bristol, UK

* Joint senior authors.

Keywords HUNT, Developmental Origin of Health and Disease, Birthweight, cardiometabolic disease

There is a robust and well-documented observational relationship between lower birthweight and higher risk of cardiometabolic disease in later life. We investigated whether there was evidence for causal effects of the intrauterine environment, as proxied by maternal SNPs that influence offspring birthweight independent of offspring genotype, on offspring cardiometabolic risk factors. We investigated whether a genetic risk score of maternal SNPs associated with offspring birthweight was also associated with offspring cardiometabolic risk factors, after controlling for offspring genotypes at the same loci, in up to 45,849 parent-offspring pairs from the Nord-Trøndelag Health (HUNT) Study. We implemented a computationally efficient genetic linear mixed model using the OpenMx software package, which allowed us to take into account the considerable cryptic relatedness in the HUNT study, making maximum use of the data. We found little evidence for a maternal genetic effect of birthweight associated variants on offspring cardiometabolic risk factors after adjusting for offspring genotypes at the same loci. Likewise, we 
found little evidence for paternal genetic effects on offspring cardiometabolic risk factors performing similar analyses in fatheroffspring pairs. In contrast, offspring genetic risk scores of birthweight-associated variants were strongly related to many cardiometabolic risk factors, even after conditioning on maternal genotypes at the same loci. Our results suggest that the maternal intrauterine environment, as proxied by maternal SNPs that influence offspring birthweight, is unlikely to be a major determinant of adverse cardiometabolic outcomes. In contrast, genetic pleiotropy in the offspring genome explains some of the observational relationship between birthweight and cardiometabolic risk.

Grant Support: Support for this research have been given by the Norwegian Diabetes Association and Nils Normans minnegave. G.H.M is supported by the Norwegian Research Council (Post doctorial mobility research grant 287198). N.M.W. is supported by an Australian National Health and Medical Research Council Early Career Fellowship (APP1104818). MCN and OpenMx development were funded by NIH grant DA-018673. D.A.L is supported by the British Heart Foundation (AA/18/7/34219) and European Research Council (669545). D.A.L, G.D.S, D.M.E are affiliated with a Unit that receives support from the University of Bristol and the UK Medical Research Council (MC_UU_00011/1 and MC_UU_00011/6). D.M.E. is funded by an Australian National Health and Medical Research Council Senior Research Fellowship (APP1137714) and NHMRC project grants (GNT1125200, GNT1157714, GNT1183074). RMF is supported by a Wellcome Trust and Royal Society Sir Henry Dale Fellowship (104150/Z/14/Z).

\section{Distinguishing dimensions of social withdrawal in childhood: Polygenic score differently predict trajectories of social wariness and preference for solitude from age 6 to 12 years}

Geneviève Morneau-Vaillancourt ${ }^{1}$, Stéphane Paquin ${ }^{1,2}$, Till Andlauer $^{3}$, Éloi Gagnon ${ }^{1}$, Isabelle Ouellet-Morin ${ }^{4}$, Mara R Brendgen ${ }^{5}$, Frank Vitaro ${ }^{6}$, Richard E Tremblay ${ }^{7}$, Sylvana Côté ${ }^{8}$, Jean-Philippe Gouin $^{9}$, Jean Séguin ${ }^{10}$, Ginette Dionne ${ }^{1}$, Michel Boivin ${ }^{1}$

${ }^{1}$ École de psychologie, Université Laval, Québec, Québec, Canada

${ }^{2}$ Department of Psychology, The Pennsylvania State University, Pennsylvania, United States

${ }^{3}$ Department of Neurology, Klinikum rechts der Isar, Technical University of Munich, Germany

${ }^{4}$ École de criminologie, Université de Montréal, Montréal, Canada

${ }^{5}$ Département de psychologie, Université du Québec à Montréal, Montréal, Canada

${ }^{6}$ École de psychoéducation, Université de Montréal, Montréal, Canada

${ }^{7}$ Département de psychologie, Université de Montréal, Montréal, Canada

${ }^{8}$ École de santé publique, Université de Montréal

${ }^{9}$ Department of Psychology, Concordia University

${ }^{10}$ Faculté de médecine, Université de Montréal

Keywords Social Withdrawal, Polygenic Scores, Social Wariness, Preference For Solitude, Longitudinal Study.

Chronic social withdrawal in childhood is associated with socioemotional problems, such as loneliness and peer exclusion (BoothLaForce and Oxford 2008). However, as social withdrawal is multifaceted, it is crucial to distinguish social wariness from preference for solitude in examining developmental pathways. These two dimensions of social withdrawal vary in their affective and motivational underpinnings, and possibly have different genetic and environmental etiology. While persisting social withdrawal is predominantly accounted for by genetic factors (Hoekstra et al. 2008), it remains unclear whether the development of social wariness and preference for solitude is explained by similar or distinct genetic factors.

The objective is to examine if and how polygenic scores of known traits associated with social withdrawal (depression, loneliness, subjective well-being, ADHD, ASD) uniquely and jointly predict trajectories of social wariness and preference for solitude from age 6 to 12 years.

Teacher-rated measures for social wariness and preference for solitude were collected at five occasions between 6 and 12 years for 1406 genotyped participants from two prospective longitudinal studies in Quebec, Canada. Latent class growth analyses were conducted to identify longitudinal trajectories, which were then predicted by polygenic scores in multinomial regressions.

A high-chronic preference for solitude was modesty predicted by each individual polygenic score, as well as by the common genetic variation shared among them. In contrast, high-chronic social wariness was only modestly predicted by the loneliness polygenic score.

These findings support a genetic distinction between these two dimensions of social withdrawal.

\section{References}

Booth-LaForce, C., \& Oxford, M. L. (2008). Trajectories of social withdrawal from grades 1 to 6: Prediction from early parenting, attachment, and temperament. Developmental Psychology, 44, 1298-1313. https://doi.org/10.1037/a0012954

Hoekstra, R. V., Bartels, M., Hudziak, J. J., Van Beijsterveldt, T. C. E. M., \& Boomsma, D. I. (2008). Genetic and environmental influences on the stability of withdrawn behavior in children: A longitudinal, multi-informant twin study. Behavior Genetics, 38, 447-461. https://doi.org/10.1007/s10519-008-9213-4.

Grant Support: Doctoral research scholarships from Fonds de recherche du Québec - Société et Culture (FRSC) and the Quebec Network on Suicide, Mood Disorders, and Related Disorders (Geneviève Morneau-Vaillancourt). Canada Research Chair in Child Development (Michel Boivin). Fonds de recherche du Québec - Santé (FRQS) through the Quebec Network on Suicide, Mood Disorders and Related Disorders. Social Science and Humanities Research Council (SSHRC)

FRQSC. National Health Research Development Program. Canadian Institutes for Health Research (CIHR). Université Laval. Université de Montréal. Québec Government's Ministry of Health, Ministry of Education, and Ministry of Family Affairs. Lucie and André Chagnon Foundation. Robert-Sauvé Research Institute of Health and Security at Work. Québec Statistics Institute. St-Justine Research Centre

\section{Would you like to know? The relationship between aversion towards ambiguity and extreme worry about finding out your own genetic predisposition for mental health problems}

Jose J. Morosoli ${ }^{1,2}$; Lucía Colodro-Conde ${ }^{1}$; Fiona K. Barlow ${ }^{2}$; Sarah E. Medland ${ }^{1,2}$

${ }^{1}$ Psychiatric Genetics, QIMR Berghofer Medical Research Institute, QLD Australia,

${ }^{2}$ School of Psychology, University of Queensland, QLD Australia

Keywords Need For Closure, Coping, Communication, Genetic Predisposition, Counseling

Learning about our own and others' genetic predisposition for health conditions can have significant impact on someone's life. The public 
is already learning about their predisposition for hundreds of complex traits via direct-to-consumer genetic testing, but their ability to understand and cope with such information is a contentious issue.

We surveyed over 5000 individuals in Australia, UK, and US, on their beliefs about genetic predisposition for alcohol dependence, schizophrenia, and depression, genetic literacy, and several psychosocial variables. We assessed 'extreme worry' about genetics, which involves: i) only wanting to know about genetic risk if there is something that can be done about the condition, ii) worrying about not being able to cope with the information, iii) not wanting to have children if they had a high genetic predisposition, and iv) not wanting to choose a partner who had a high genetic predisposition for the condition.

Across all samples and traits, the strongest predictor for extreme worry is 'need for closure', an individual's desire for firm answers and aversion towards uncertainty. Need for closure emerges as key for understanding how people make sense of complex issues, however little is known about its development across the lifespan, its genetic correlates, or its role in counseling. Using a twin design, we estimated genetic and environmental contributions to the phenotype (VA = $37.6 \%$ [26.0-47.8], $\mathrm{VE}=62.4 \%$ [52.2-74.0]). There was no evidence of moderation of variance components by age or polygenic scores for educational attainment. Detailed results and implications for communication about genetics will be discussed.

Grant Support: This study was funded by the John Templeton Foundation (Genetics and Human Agency Project).

\section{Latent genetic structure of sleep health and psychopathology}

\author{
Claire L Morrison ${ }^{1,2}$, Evan A Winiger ${ }^{1,2}$, Céline Vetter $^{3}$, Naomi P \\ Friedman ${ }^{1,2}$ \\ ${ }^{1}$ Department of Psychology and Neuroscience, University of Color- \\ ado Boulder, 1905 Colorado Ave, Boulder, CO 80309 USA. \\ ${ }^{2}$ Institute for Behavioral Genetics, University of Colorado Boulder, \\ $148030^{\text {th }}$ Street, Boulder, CO 80309 USA \\ ${ }^{3}$ Department of Integrative Physiology, 1725 Pleasant Street, Boul- \\ der, CO 80309 USA.
}

Keywords Genomic Structural Equation Modeling, Internalizing, Externalizing, Sleep Health, Insomnia

Sleep health is a construct that has gained popularity in recent literature. It is a multi-faceted construct that includes subjective satisfaction with sleep, alertness during waking hours, adequate sleep duration, timing, regularity and efficiency. These components of sleep health are considered independent and have been shown to be uniquely associated with health and wellbeing outcomes. Sleep health components can be measured subjectively (by self-report or sleep diaries), or objectively (often by actigraphy or physiological data). Here, we present the first study to examine the genetic structure of sleep health and its relationship to psychopathology. We used Genomic structural equation modeling (SEM) to create latent factors of alertness (subjective daytime sleepiness and objective daytime inactivity), efficiency (objective efficiency and number of sleep episodes), sleep dissatisfaction/disorder (self-reported short sleep $[<6$ $\mathrm{h} /$ night] and insomnia) and timing (self-reported chronotype and objective sleep midpoint). We then assessed relationships of these sleep health latent factors with each other and latent psychopathology factors. Alertness, efficiency and dissatisfaction were most strongly genetically associated with psychopathology, in that genetic liability for being less alert during waking hours, having lower efficiency and more sleep dissatisfaction correlated with higher genetic liability for internalizing and externalizing psychopathology. Timing did not significantly associate with any psychopathology factors. Interestingly, a thought disorder factor of anorexia and OCD had a negative genetic correlation with daytime alertness. These correlations imply the unique facets of sleep health are genetically separable and exhibit distinct patterns of associations with psychopathology.

Grant Support: National Institute of Mental Health Grant MH016880

\section{Genetics, parenting and family functioning: what drives the development of self-regulation from adolescence to adulthood?}

Ida M. Mueller ${ }^{1}$, Frank M. Spinath ${ }^{1}$, Malte Friese ${ }^{1}$, Elisabeth Hahn ${ }^{1}$ ${ }^{1}$ Department of Psychology, Saarland University, Saarbruecken, Saarland, Germany

Keywords Self-Regulation, Personality, Cognition, Development, Family Functioning

Self-regulatory competencies develop early in childhood and play a meaningful role in predicting crucial life outcomes. Former research has already specified relevant genetic and environmental influences while primarily investigating early childhood development. In the present study, we used a cross-sectional multi-cohort design to investigate self-regulation from adolescence to early adulthood examining the relative contribution of genetic and environmental influences with a special focus on family environments. Regarding potential environmental influences, previous studies often neglected that parental resources may be distributed differentially between children. Therefore, we investigated whether parenting style and chaotic home environments act as shared and/or non-shared environment on self-regulation. We used data from the German twin family study TwinLife $(\mathrm{N}=3354)$. Twins aged 13,19 and 25 years rated their self-regulation, perceived parenting behavior and home environment. Analyses revealed increasing mean-level and one-year stability of self-regulation across ages. Particularly from age 19 to 25, genetic influences on self-regulation increased (47 to $73 \%$ ) while nonshared environmental influences decreased, possibly due to amplifying genetic effects or gene-environment correlation. Furthermore, while CHAOS and negative parenting went along with lower selfregulation, twin difference models revealed that differences in CHAOS and negative parenting directly predicted differences in selfregulation controlled for genetic and environmental similarities. In contrast, the positive relation between positive parenting and selfregulation was mediated through shared genetic and environmental influences. Our results suggest further developmental processes of genetic and environmental influences after childhood and will be discussed referring to the main theories of self-regulation and differential environmental influences within families.

Grant Support: This research was supported by a grant from the German Research Foundation awarded to Martin Diewald (DI 759/11-1), Rainer Riemann (RI 595/8-1), and Frank M. Spinath (SP 610/6-1).

\section{Shared genetic effects between schizophrenia and substance abuse: a multiplex extended pedigree study}

Christie W. Musket, M.S. ${ }^{1}$, Petra E. Rupert, B.S. ${ }^{1}$, Susan S. Kuo, M.S., ${ }^{1,2}$, David R. Roalf, Ph.D. ${ }^{3}$, Konasale Prasad, M.D. ${ }^{4}$, Joel Wood $^{4}$, Ruben C. Gur, Ph.D. ${ }^{3}$, Laura Almasy, Ph.D. ${ }^{5}$, Raquel E. Gur, 
M.D., Ph.D. ${ }^{3}$, Vishwajit L. Nimgaonkar, M.D., Ph.D. ${ }^{4}$, Michael F. Pogue-Geile, Ph.D. ${ }^{1}$

${ }^{1}$ University of Pittsburgh, Department of Psychology, Pittsburgh, PA, USA;

${ }^{2}$ University of California, Los Angeles, Department of Psychiatry, Los Angeles, CA;

${ }^{3}$ University of Pennsylvania, Department of Psychiatry, Philadelphia, PA, USA;

${ }^{4}$ University of Pittsburgh, Department of Psychiatry, Pittsburgh, PA, USA;

${ }^{5}$ University of Pennsylvania, Department of Genetics, Philadelphia, PA, USA

Keywords Schizophrenia; Substance Abuse; Genetic Correlation; Extended Pedigree

Substance abuse is a serious public health issue and is increased among those with schizophrenia (28-47\% versus $14.6 \%$ in the general population). In an attempt to better understand this comorbidity, the current study used an extended pedigree sample ascertained through schizophrenia probands (total sample $\mathrm{N}=1307$, with $\mathrm{N}=790$ relatives and $\mathrm{N}=517$ unrelated controls) to examine the degree to which genetic effects influence shared liability for schizophrenia $(\mathrm{N}=123)$ and three different categorizations of DSM-IV substance use diagnoses: any substance dependence or abuse diagnosis $(\mathrm{N}=251)$, cannabis dependence or abuse $(\mathrm{N}=106)$ and alcohol dependence or abuse $(\mathrm{N}=179)$. We also assessed shared genetic effects between schizophrenia and two measures of nicotine use: binary "ever smoked daily" status (total available $\mathrm{N}=1082$; smokers $\mathrm{N}=532$ ) and a continuous measure of "pack years" (total available $\mathrm{N}=1047$; pack years $>0 \mathrm{~N}=489$ ). The univariate heritabilities of any substance dependence/abuse diagnosis $\left(\mathrm{h}^{2}=0.601, p=2.052 \mathrm{e}^{-8}\right)$, cannabis dependence/abuse $\left(\mathrm{h}^{2}=0.926, p=2.083 \mathrm{e}^{-9}\right)$, and alcohol dependence/abuse $\left(\mathrm{h}^{2}=0.434, p=1.00 \mathrm{e}^{-4}\right)$, smoking status $\left(\mathrm{h}^{2}=0.518, p=\right.$ $\left.1.00 \mathrm{e}^{-7}\right)$, and pack years $\left(\mathrm{h}^{2}=0.873, p=1.360 \mathrm{e}^{-36}\right)$ were all significant. The genetic correlations between schizophrenia and any substance dependence/abuse $\left(\mathrm{R}_{\mathrm{g}}=0.300, p=0.040\right)$, schizophrenia and alcohol dependence/abuse $\left(\mathrm{R}_{\mathrm{g}}=0.389, p=0.024\right)$, and schizophrenia and pack years of cigarette smoking $\left(\mathrm{R}_{\mathrm{g}}=0.458, p=\right.$ 0.006) were significant; however, the genetic relationship between schizophrenia and cannabis dependence/abuse $\left(\mathrm{R}_{\mathrm{g}}=0.190, p=0.124\right)$ and schizophrenia and smoker status $\left(\mathrm{R}_{\mathrm{g}}=0.197, p=0.167\right)$ were not significant. Environmental correlations were all non-significant. Results indicate that genetic effects play a significant role in both unique and shared variance between schizophrenia and multiple measures of substance abuse. Future research will evaluate the genetic mediating effects of MRI brain structure volumes between schizophrenia and substance abuse.

Grant Support: NIMH collaborative R01s, MH42191 (REG), MH63480 (VLN); and MH60722 (LA), T32 GM81760-10 (CWM), and CIHR Doctoral Foreign Study Award (SSK)

\section{Heritability of serum brain-derived neurotrophic factor (BDNF) in twins, siblings and adoptees}

Chloe Myers ${ }^{1}$, Andrew Smolen ${ }^{2}$, Sally J. Wadsworth ${ }^{2}$, Chandra A. Reynolds ${ }^{1}$

${ }^{1}$ Department of Psychology, University of California - Riverside, Riverside, California USA

${ }^{2}$ Institute for Behavioral Genetics, University of Colorado Boulder, Boulder, Colorado, USA

Keywords Brain-derived neurotrophic factor, heritability, cognition, exercise
Brain-derived neurotrophic factor (BDNF) is a nerve growth factor involved in neuronal processes necessary for learning and memory. BDNF variants, principally the Val66Met variant, as well as circulating levels of BDNF have been evaluated in terms of their association with cognitive performance and decline and possible mediating pathways including BMI and exercise. The heritability of serum BDNF levels has received little attention outside of two familybased studies reporting estimates of .20-.48. We assessed the heritability of serum BDNF in participants from the ongoing Colorado Adoption/Twin Study of Lifespan behavioral development and cognitive aging (CATSLife). CATSLife includes participants from two foundational studies, the Colorado Adoption Project (CAP) and Longitudinal Twin Study (LTS). Serum BDNF was available for 1009 participants representing 619 sibships (158 MZ, 158 DZ, 160 Controls, 143 Adoptive). The average age of participants was 33.04 years $(\mathrm{SD}=4.85)$, with $53 \%$ females. Intraclass correlations by family type suggested strong heritable components adjusted for age, sex, batch, and fasting: $\mathrm{MZ}=.48, \mathrm{DZ}=.17$, Control $=.11$, Adoptive $=.08$. An ADCE biometrical model was fitted to twins and sibling trios where the inclusion of adoptees, full-siblings and twins allowed for estimation of both dominance and shared environment. Full model results suggested additive genetic influences $(A)=20.5 \%$, dominance $(D)=$ $28.6 \%$, shared environment $(\mathrm{C})=1.4 \%$ and nonshared environment (E) $=49.5 \%$. Findings suggest that about half of the variance is explained by genetic contributions with the remainder influenced by person-specific environmental influences. Whether BDNF levels show shared etiology with health and cognitive phenotypes in CATSLife remains to be examined.

Grant Support: NIA R01AG046938.

\section{Associations between anxiety symptoms and health- related quality of life: a population-based twin study in Sri Lanka}

Zeynep Nas ${ }^{1}$, Helena M. S. Zavos ${ }^{2}$, Athula Sumathipala, ${ }^{3,7}$, Kaushalya Jayaweera $^{3}$, Sisira Siribaddana ${ }^{4}$, Matthew Hotopf ${ }^{5,6}$, Frühling V. Rijsdijk $^{1}$

${ }^{1}$ Social, Genetic \& Developmental Psychiatry Centre, Institute of Psychiatry, Psychology and Neuroscience, King's College London, London, UK

${ }^{2}$ Department of Psychology, Institute of Psychiatry, Psychology and Neuroscience, King's College London, London, UK

${ }^{3}$ Institute for Research and Development, Colombo, Sri Lanka

${ }^{4}$ Faculty of Medicine \& Allied Sciences, Rajarata University of Sri Lanka, Anuradhapura, Sri Lanka

${ }^{5}$ Psychological Medicine Department, Institute of Psychiatry, Psychology, and Neuroscience, King's College London, London, UK

${ }^{6}$ NIHR Biomedical Research Centre for Mental Health at the South London and Maudsley NHS Foundation Trust, King's College London, London, UK

${ }^{7}$ Research Institute for Primary Care and Health Sciences, Faculty of Health, Keele University, Keele, UK

Keywords Anxiety, Quality Of Life, Twin Study, Sri Lanka

Anxiety not only influences mental wellbeing but also negatively impacts other areas of health including physical wellbeing and quality of life. Yet, there is limited research on (a) the genetic and environmental aetiology of such relationships; (b) sex differences in their aetiological relationship and (c) how this works/exists in non-western populations. In this talk, I will present how we addressed these research gaps using data from the Colombo Twin and Singleton Study (CoTASS), a population-based sample in Sri Lanka. Using bivariate 
genetic model fitting, I find significant sex differences in both the variance and covariance of anxiety and health-related quality of life. I will discuss these results as well as follow with my plans for future directions.

\section{Effects of non-random ascertainment on variance component estimates from classical twin studies}

\author{
Michael C. Neale ${ }^{1}$ \\ ${ }^{1}$ Department of Psychiatry, Virginia Institute for Psychiatric and \\ Behavioral Genetics, Richmond, Virginia, USA \\ Keywords Ascertainment, simulation, twins, heritability, bias
}

Most twin studies involve some degree of voluntary participation. Research studies often have difficulty ascertaining participants from, e.g., low socioeconomic levels, but it is not clear what effects such non-random ascertainment would have on estimates of genetic and environmental variance components. Simulation studies were conducted to explore this potential source of bias. If twin pairs are discarded when at least one participant is below a particular threshold, non-linear effects on trait heritability estimates are seen, with an increase in apparent heritability from .8 to .9 with between $1 \%$ and $8 \%$ attrition, which falls to nominal levels at $\sim 16 \%$ attrition. Similar but somewhat attenuated patterns are observed if individuals are retained if their score is above threshold, but their cotwin's is below (i.e., unmatched twins are included). In either case, common environment variation drops monotonically, becoming negative when selection omits $\sim 7 \%$ or more of the sample. Implications for the study of variation among human populations include sampling methodology and the relative merits of alternative approaches to estimating heritability.

Grant Support: R01DA-018673, U01DA051037A

\section{Exploring puberty as a moderator of genetic and environmental influences on body weight and shape concerns in females}

Shannon M. O'Connor ${ }^{1}$, Kristen M. Culbert ${ }^{2}$, Laura A. Mayhall ${ }^{3}$, S. Alexandra Burt ${ }^{4}$, \& Kelly L. Klump ${ }^{4}$

${ }^{1}$ Department of Psychiatry and Behavioral Neuroscience, University of Chicago, Chicago, Illinois, USA

${ }^{2}$ Department of Family Medicine and Public Health, Wayne State University School of Medicine, Detroit, MI, USA

${ }^{3}$ Department of Communicative Sciences and Disorders, Michigan State University, East Lansing, MI, USA

${ }^{4}$ Department of Psychology, Michigan State University, East Lansing, MI, USA

Keywords Body Dissatisfaction; Weight Preoccupation; Eating Disorders; Genetic; Puberty

The heritability of disordered eating increases during puberty; however, prior studies have largely examined a composite score of disordered eating, rather than specific symptoms (e.g., Culbert et al. 2009; Klump et al. 2007a, b). Body weight and shape concerns cut across all eating disorder diagnoses and are some of the strongest prospective risk factors for the development of eating disorders (Jacobi 2005; Killen et al. 1996; Stice and Shaw 2002). Yet, little is known about potential developmental increases or decreases in genetic and environmental influences for these key symptoms. This study examined differences in genetic and environmental effects on a range of body weight and shape concerns during puberty and compared results to findings for an overall disordered eating composite score. Participants were 926 same-sex female twins (ages 8-16) from the Michigan State University Twin Registry. Well-validated questionnaires were used to examine pubertal maturation, overall disordered eating, and a range of cognitive body weight/shape constructs: body dissatisfaction, weight/shape concerns, and weight preoccupation. Findings for overall disordered eating were similar to previous work, with significantly increased genetic effects in girls at more advanced pubertal development. Importantly, these same pubertal increases in genetic influences were observed for body dissatisfaction and weight/shape concerns. However, no pubertal moderation of genetic effects was observed for weight preoccupation; instead, pubertal moderation of nonshared and shared environmental effects was observed. Findings point to differences in the extent to which genetic and environmental factors contribute to various cognitive body weight and shape symptoms during puberty.

\section{References}

Culbert, K. M., Burt, S. A., McGue, M., Iacono, W. G., \& Klump, K. L. (2009). Puberty and the genetic diathesis of disordered eating attitudes and behaviors. Journal of Abnormal Psychology, 118(4), 788-796.

Jacobi, C. (2005). Psychosocial risk factors for eating disorders. In Eating Disorders Review (ed. S. A. Wonderlich, J. E. Mitchell, M. de Zwaan, \& Steiger, H.), pp. 59-85. Radcliffe Publishing Ltd: Oxford, UK.

Killen, J. D., Taylor, C. B., Hayward, C., Haydel, K. F., Wilson, D. M., Hammer, L., Kraemer, H., et al. (1996). Weight concerns influence the delveopment of eating disorders: A 4-year prospective study. Journal of Consulting and Clinical Psychology, 64(5), 936-940.

Klump, K. L., Burt, S. A., McGue, M., \& Iacono, W. G. (2007a). Changes in genetic and environmental influences on disordered eating across adolescence: A longitudinal twin study. Archives of General Psychiatry, 64(12), 1409-1415.

Klump, K. L., Perkins, P., Burt, S. A., McGue, M., \& Iacono, W. G. (2007b). Puberty moderates genetic influences on disordered eating. Psychological Medicine, 37(5), 627-634.

Stice, E., \& Shaw, H. E. (2002). Role of body dissatisfaction in the onset and maintenance of eating pathology: A synthesis of research findings. Journal of Psychosomatic Research, 53(5), 985-993.

Grant Support: This work was supported by the National Institute of Mental Health [USA; R01 MH082054 awarded to Drs. Kelly Klump and S. Alexandra Burt; T32 MH082761 awarded to Dr. Shannon O'Connor].

\section{Epigenetics and handedness}

Veronika V. Odintsova $^{1}$, Matthew Suderman ${ }^{2}$, Fiona A. Hagenbeek ${ }^{1}$, Doretta Caramaschi ${ }^{2}$, Jouke-Jan Hottenga ${ }^{1}$, Lannie R.S. Ligthart ${ }^{1}$, Catharina E.M. van Beijsterveld ${ }^{1}$, Gonneke Willemsen ${ }^{1}$, Erik A. Ehli $^{3}$, Gareth E. Davies ${ }^{3}$, Eco de Geus ${ }^{1}$, Caroline Relton ${ }^{2}$, Dorret I. Boomsma ${ }^{1}$, Jenny van Dongen ${ }^{1}$

${ }^{1}$ Department of Biological Psychology, Vrije University Amsterdam, Amsterdam, The Netherlands

${ }^{2}$ MRC Integrative Epidemiology Unit, Bristol Medical School, Population Health Science, University of Bristol, Bristol, UK

${ }^{3}$ Avera Institute for Human Genetics, Sioux Falls, South Dakota, USA

Keywords Handedness, Epigenome-Wide Association Studies, Methylation Scores

The etiology of handedness has been investigated extensively in terms of heritability and in relationship with behavioral and neurological 
traits and yet remains largely unexplained. The heritability of handedness is relatively low: additive genetic effects explained about $25 \%$ of the variance in handedness in large-scale twin studies (Medland et al. 2009). A genome-wide association study that included more than 1.5 million right-handed and 194 thousand left-handed individuals found 42 genetic loci associated with left-handedness (Partida et al. 2019). To investigate this mostly non-genetic trait, we performed an epigenome-wide association study in peripheral tissues (buccal cells and blood) and at different ages from birth to adulthood, combining data from two large cohorts: the Netherlands Twin Register (NTR) children $(\mathrm{N}=946)$ and adults $(\mathrm{N}=2682)$ and the Avon Longitudinal Study of Parents and Children (ALSPAC) children $(\mathrm{N}=729)$ and parents $(\mathrm{N}=1232)$. No meta-analyzed associations of the NTR and ALSPAC adults survived adjustment for multiple testing (Bonferroni-adjusted $\mathrm{p}<0.05$ ). Top CpGs were associated with ATP6V1B2, LRRC2, CKAP4, C13orf18, RING1 genes. Several differentially methylated regions were associated with handedness in both buccal cells and blood in children. Created DNA methylation scores of handedness explained little variance in children and more less in adults.

\section{References}

1. Medland, S. E. et al. Genetic influences on handedness: data from 25,732 Australian and Dutch twin families. Neuropsychologia 47, 330-337 (2009).

2. Partida, G. C. et al. Genome-wide association study identifies 49 common genetic variants associated with handedness. bioRxiv 831321 (2019).

Grant Support: Amsterdam Public Health Institute travel grant VVO; KNAW Academy Professor Award (PAH/6635) to DIB.

The UK Medical Research Council and Wellcome (Grant ref: $102215 / 2 / 13 / 2$ ) and the University of Bristol provide core support for ALSPAC. A comprehensive list of grants funding is available on the ALSPAC website (http://www.bristol.ac.uk/alspac/external/documen ts/grantacknowledgements).

pdf). This research was specifically funded by the BBSRC (grant numbers BBI025751/1 and BB/I025263/1). C.R. and D.C. are funded by the MRC (grant numbers MC_UU_00011/5 and MC_UU_00011/ 1). "Aggression in Children: Unraveling gene-environment interplay to inform Treatment and InterventiON strategies" project (ACTION; BBMRI -NL: Biobanking and Biomolecular Resources Research Infrastructure grant $\mathrm{nr}$ 184.021.007 and 184.033.111; Eu-FP7 grant agreement no 602768). Netherlands Twin Registry Repository NWO 480-15-001/674: Netherlands Organization for Scientific Research (NWO). JvD is supported by the NWO-funded X-omics project (184.034.019).

\section{Genetic and environmental influences on sexual orientation: Moderation by childhood gender nonconformity and early-life adversity}

Olakunle Ayokunmi Oginni ${ }^{1}$, Katarina Alanko ${ }^{2}$, Patrick Jern ${ }^{2}$, Frühling Vesta Rijsdijk ${ }^{1}$

${ }^{1}$ The Social, Genetic and Developmental Psychiatry centre, Institute of Psychiatry, Psychology and Neuroscience, Denmark Hill, King's College London, ${ }^{2}$ Department of Psychology, Ảbo Akademi University, FIN-20500, Åbo, Finland.

Keywords Sexual Orientation, Childhood Gender Nonconformity, Early-Life Adversity, Moderation

Existing evidence indicates genetic and non-genetic influences on sexual orientation; however (Bailey et al. 2016), the possibility of gene-environment interplay has not been previously formally tested despite theories indicating this (Rind 2013; Bem 1996). This study investigated whether childhood gender nonconformity and early-life adversities independently moderated individual differences in sexual orientation and the relationship between sexual orientation and childhood gender nonconformity in a Finnish twin cohort. It also investigated the etiological bases of the proposed moderation effects. Sexual orientation, childhood gender nonconformity and early-life adversities were assessed using standardized questionnaires. Twin modelling was carried out using Structural Equation Modelling in OpenMx (Boker et al. 2011). The present study showed that childhood gender nonconformity and early-life adversities were significantly associated with increased individual differences in sexual orientation ( $\beta=0.14$ and 0.18 respectively, 95\% CIs: $0.13-0.21$ ) and covariance between sexual orientation-childhood gender nonconformity ( $\beta=0.06$ and 0.08 respectively, $95 \%$ CIs: $0.04-0.11$ ). Moderation of individual differences in sexual orientation was explained by genetic ( $\beta \mathrm{A}=0.50$ for childhood gender nonconformity, 95\% CI: 0.45-0.56) and individual-specific environmental influences $(\beta E=0.07$ and 0.10 for childhood gender nonconformity and earlylife adversities respectively, 95\% CIs: 0.02-0.17) while moderation of the relationship between sexual orientation and childhood gender nonconformity was driven by individual-specific environmental influences $(\beta E=0.15$ and 0.10 for childhood gender nonconformity and early-life adversities respectively, 95\% CIs: 0.04-0.21). These findings suggest that etiological (genetic and environmental) influences on sexual orientation are dynamic and are in turn influenced by developmental factors.

References

Bailey, J. M., Vasey, P. L., Diamond, L. M., Breedlove, S. M., Vilain, E., \& Epprecht, M. (2016). Sexual orientation, controversy, and science. Psychological Science in the Public Interest, 17, 45-101. doi: https://doi.org/10.1177/1529100616637616

Bem, D. J. (1996). Exotic becomes erotic: A developmental theory of sexual orientation. Psychological Review, 103, 320-335. https://dx.doi.org/10.1037/0033-295X.103.2.320

Boker, S., Neale, M., Maes, H., Wilde, M., Spiegel, M., Brick, T., . . . Bates, T. (2011). OpenMx: An open source extended structural equation modeling framework. Psychometrika, 76, 306-317. https://doi.org/10.1007/S11336-010-9200-6

Rind, B. (2013). Homosexual orientation-From nature, not abuse: A critique of Roberts, Glymour, and Koenen. Archives of Sexual Behavior, 42, 1653-1664. https://dx.doi.org/10.1007/s105 08-013-0080-6

\section{Evaluation of polygenic prediction methodology within a reference-standardized framework}

Oliver Pain ${ }^{1,2}$, Kylie P. Glanville ${ }^{1}$, Saskia Hagenaars ${ }^{1}$, Saskia Selzam $^{1}$, Anna E. Fürtjes ${ }^{1}$, Helena Gaspar ${ }^{1}$, Jonathan R. I. Coleman ${ }^{1}$, Kaili Rimfeld ${ }^{1}$, Gerome Breen ${ }^{1,2}$, Robert Plomin ${ }^{1}$, Lasse Folkersen ${ }^{2}$, Cathryn M. Lewis ${ }^{1,2,3}$.

${ }^{1}$ Social, Genetic and Developmental Psychiatry Centre, Institute of Psychiatry, Psychology and Neuroscience, King's College London, London, SE5 8AF, United Kingdom

${ }^{2}$ NIHR Maudsley Biomedical Research Centre, South London and Maudsley NHS Trust, London, SE5 8AF, UK.

${ }^{3}$ Institute of Biological Psychiatry, Sankt Hans Hospital, Copenhagen, 4000 Roskilde, Denmark

${ }^{4}$ Department of Medical and Molecular Genetics, Faculty of Life Sciences and Medicine, King's College London, London, SE1 9RT, UK. 
Keywords polygenic scores, reference-standardized, prediction, elastic net, GWAS

The predictive utility of polygenic scores is increasing but it is unclear which polygenic scoring method performs best. It is often advantageous to calculate polygenic scores using a reference-standardized framework, using a common set of variants and reference-estimated linkage disequilibrium and allele frequencies. This study evaluates the predictive utility of several polygenic scoring methods within a reference-standardized framework.

The following methods are evaluated using anthropometric, neuropsychiatric and complex disease outcomes measured in UK Biobank and Twins Early Development Study (TEDS): p-value thresholding and clumping (pT+clump), SBLUP, lassosum, LDPred, PRScs and SBayesR. Strategies to identify optimal p-value threshold and shrinkage parameters are compared, including 10-fold cross validation, pseudovalidation (no validation sample), and multi-polygenic score elastic net models.

Using 10-fold cross-validation to identify the most predictive p-value threshold or shrinkage parameter, lassosum, PRScs and LDPred provided optimal prediction (relative improvement of $14-17 \%$ over pT+clump). Using pseudovalidation to optimize the polygenic score, the best method was PRScs, with a relative improvement of $>11 \%$ over other pseudovalidation methods (lassosum, SLBLUP, SBayesR, LDPred), and only $1 \%$ worse than the best polygenic score identified by 10 -fold cross validation. Elastic net models containing polygenic scores based on a range of parameters consistently improve prediction over any single polygenic score.

Within a reference-standardized framework, the best polygenic prediction was achieved using lassosum, PRScs, modelling multiple polygenic scores that are derived using a range of parameters. As polygenic scores are widely applied in research studies, users should be aware of differences in prediction across methods.

Grant Support Funded by the MRC (MR/N015746/1), and the National Institute for Health Research (NIHR) Biomedical Research Centre at South London and Maudsley NHS Foundation Trust and King's College London.

\section{Modifiable risk factors and miscarriage: a Mendelian Randomization analysis}

Jodie N Painter $^{1}$, Nick G Martin ${ }^{2}$, Sarah E Medland ${ }^{1}$

${ }^{1}$ Psychiatric Genetics Laboratory, QIMR Berghofer Medical Research Institute, Brisbane, Queensland, Australia

2 Genetic Epidemiology Laboratory, QIMR Berghofer Medical Research Institute, Brisbane, Queensland, Australia

Keywords Miscarriage, coffee, smoking, alcohol, adiposity

Miscarriage, the loss of an embryo or fetus before 20 weeks of gestation, is estimated to end between $10-25 \%$ of clinically confirmed pregnancies. While approximately $50 \%$ of these miscarriages have been shown to be due to chromosomal abnormalities, observational studies have suggested various modifiable lifestyle factors may also increase miscarriage risk. Consequently, health guidelines typically suggest that prospective parents eliminate, limit or modify these factors, particularly the consumption of coffee and alcohol, smoking, and adiposity, before and during pregnancy. However, definitive causal relationships between these risk factors and miscarriage have not yet been demonstrated.

Here, we present a genome-wide association and Mendelian randomization analysis including $>200,000$ women in the UK Biobank and two QIMR Berghofer samples to investigate potential causal associations between miscarriage and the four most-often cited miscarriage risk factors-the consumption of coffee and alcohol, smoking and increased body mass index. Using the most significant genetic variants from recent genome-wide association studies for these factors as instrumental variables we find evidence for a causal relationship between smoking initiation and spontaneous miscarriage (Inverse variance weighted Odds Ratio $=1.17,95 \% \mathrm{CI}=1.10-1.24, \mathrm{P}$ $=2.7 \times 10^{-7}$ ), but not for any other risk factor tested and either spontaneous or recurrent miscarriage. We suggest the significant observational associations for coffee and alcohol consumption and increased body mass index require further exploration, so as not to add to the distress or feelings of guilt and personal responsibility that are often associated with pregnancy loss.

Grant Support: National Health and Medical Research Council (Australia) Project Grant ID1163040

\section{Interplay between genetic risk and the parent environment in adolescence and substance use in young adulthood: a TRAILS study}

Joëlle A. Pasman ${ }^{1}$, Koen Smit ${ }^{1,2}$, Wilma Vollebergh ${ }^{3}$, Ilja M. Nolte, Catharina Hartman ${ }^{4}$, Abdel Abdellaoui ${ }^{5}$, Karin J.H. Verweij ${ }^{5}$, Dominique Maciejewski ${ }^{\$ 1}$, Jacqueline M. Vink ${ }^{\$ 1}$

\$Shared last author

${ }^{1}$ Behavioural Science Institute, Radboud University, Nijmegen, The Netherlands

${ }^{2}$ Centre Alcohol Policy Research, School of Psychology and Public Health, La Trobe University, Melbourne, Australia

${ }^{3}$ Faculty of Social Sciences, Utrecht University, Utrecht, The Netherlands

${ }^{4}$ Faculty of Medical Sciences, University Medical Center Groningen, Groningen, The Netherlands

${ }^{5}$ Department of Psychiatry, Amsterdam UMC, University of Amsterdam, Amsterdam, The Netherlands

Keywords gene-environment interaction, genetic nurturing, substance use, structural equation modeling, polygenic risk scores

During adolescence, many youth start using tobacco, alcohol and cannabis, a pattern that can persist into adulthood and can have deleterious health consequences. Genetic vulnerability, parent factors during adolescence, and interaction $(\mathrm{G} \times \mathrm{E})$ and correlation $(\mathrm{rGE})$ between these factors can contribute to the development of substance use.

Using 11-year prospective data from the TRacking Adolescent Individuals' Lives Survey (TRAILS, $N=1,649$ ) we modelled latent parent characteristics (ages 11-16; involvement, substance use, and parent-child relationship) in adolescents to predict young adult substance use (age 22). Based on recent GWAS, polygenic scores (PGS) were created with GCTA-SBLUP for smoking, alcohol use, and cannabis use. Using Structural Equation Modelling we modelled direct, $\mathrm{G} \times \mathrm{E}$, and $\mathrm{rGE}$ effects of parent factors and PGS on young adult smoking, alcohol use, and cannabis initiation.

High PGS, low parental involvement, high parental substance use, and low quality parent-child relationship predicted smoking. There was $\mathrm{G} \times \mathrm{E}$ such that the PGS amplified the effect of parental substance use. There was positive rGE between all parent factors and the PGS. Alcohol use was not predicted by genetic or parent factors, nor by interplay between those. Cannabis initiation was predicted by high PGS and parental substance use, but there was no evidence for $\mathrm{G} \times \mathrm{E}$ or rGE.

We found evidence for the contribution of genetic risk and parent factors to smoking and cannabis initiation, and complex interplay between those for smoking. Our method is promising for 
disentangling genetic nurturing and other complex interplay phenomena between genes and the environment.

\section{Exploring ABCD phenotypes for future behavioral genetic analysis: screen time is associated with mental health, academic outcomes, and peer relationships}

Katie N. Paulich ${ }^{1,2}$, J. Megan Ross ${ }^{1}$, Jeffrey M. Lessem ${ }^{1}$, John K. Hewitt ${ }^{1,2}$

${ }^{1}$ Institute for Behavioral Genetics, University of Colorado Boulder, Boulder, Colorado, USA.

${ }^{2}$ Department of Psychology and Neuroscience, University of Colorado Boulder, Boulder, Colorado, USA

Keywords Screen Time, Adolescence, ABCD Study, Mental Health, Behavioral Problems

We are using screens more than ever. The high rate of electronic media use among children and adolescents begs the question: is screen time harming our youth? The current study draws from a nationwide sample of 11,875 participants, aged 9 to 10 years, from the Adolescent Brain Cognitive Development (ABCD) study. We investigate relationships between screen time and mental health, behavioral problems, academic performance, sleep habits, and peer relationships, by conducting a series of correlation and regression analyses, controlling for SES and race/ethnicity. We find that more screen time is associated with worse mental health, increased behavioral problems, lower academic performance, and poorer sleep, but heightened quality of peer relationships. However, effect sizes associated with screen time and the various outcomes were small; SES was more strongly associated with each outcome measure. The initial exploration of screen time, mental health, behavioral problems, academic performance, and sleep habits in the ABCD study has laid groundwork for future analyses utilizing co-twin control designs and biometrical modeling to examine genetic and environmental contributions to those phenotypes over time.

Grant Support: Data used in the preparation of this poster were obtained from the Adolescent Brain Cognitive Development (ABCD) Study (https://abcdstudy.org), held in the National institute of Mental Health Data Archive (NDA). This is a multisite, longitudinal study designed to recruit more than 10,000 children age 9-10 and follow them over 10 years into early adulthood. The ABCD Study is supported by the National Institutes of Health (NIH) and additional federal partners under award numbers U01DA041022, U01DA041028, U01DA041048, U01DA041089, U01DA041106, U01DA041117, U01DA041120, U01DA041134, U01DA041148, U01DA041156, U01DA041174, U24DA041123, and U24DA041147. A full list of supporters is available at https://abcdstudy.org/nih-collaborators. A listing of participating sites and a complete listing of the study investigators can be found at https://abcdstudy.org/principal-investigators.html. ABCD consortium investigators designed and implemented the study and/or provided data but did not necessarily participate in analysis or writing of this report. This poster reflects the views of the authors and may not reflect the opinions or views of the NIH or ABCD consortium investigators. The $A B C D$ data repository grows and changes over time. The $A B C D$ data used in this poster came from [NIMH Data Archive Digital Object Identifier (10.15154/1503209)].
Shared genetic risk between major depression and body mass index in Han Chinese women

Roseann E. Peterson ${ }^{1,2}$, Tim B. Bigdeli ${ }^{2}$, Bradley T. Webb ${ }^{1}$, Jonathan Flint $^{3}$, Kenneth S. Kendler ${ }^{1}$

${ }^{1}$ Virginia Institute for Psychiatric and Behavioral Genetics, Department of Psychiatry, Virginia Commonwealth University, Richmond, Virginia, USA.

2 SUNY Downstate Health Sciences University, Brooklyn, New York, USA.

${ }^{3}$ Center for Neurobehavioral Genetics, Semel Institute for Neuroscience and Human Behavior, University of California Los Angeles, Los Angeles, California, USA.

Keywords Major depression, Body Mass Index, Genetic Correlation, Mendelian Randomization, Obesity

Obesity is a major public health concern and is often comorbid with psychiatric conditions including major depression (MD). Notably, several symptoms of MD are related to energy balance, including changes in appetite, weight, and sleep. Despite numerous associations between MD, BMI, and obesity there has been limited research on genetic underpinnings, particularly in diverse ancestry cohorts. Data are from the CONVERGE study of Han Chinese women with recurrent $\mathrm{MD}$ ( $n=10,640)$ using sparse whole-genome sequencing. We evaluate shared genetic liability through estimates of genetic correlation and consider the predictive value of cross-trait polygenic risk scores (PRS). To move beyond phenotype-genotype associations, we investigate causal mechanisms via Mendelian randomization. Common genetic variation accounted for $15.5 \%$ of the variance in BMI $\left(p=8.0 \times 10^{-7}\right), 22.6 \%$ in MD diagnosis $\left(P<1.0 \times 10^{-16}\right)$, and $16.1 \%$ in atypical depression $(p=0.002)$. Significant genetic correlations were seen between MD and obesity traits ranging from 0.15 for percent body fat to -0.38 for class III obesity. The BMI-PRS was negatively associated with MD case-status (effect $=-0.72, p=$ 0.014). Associations for individual variants previously associated with BMI among European populations were not robust in CONVERGE $\left(p_{F T O}=0.005\right)$. Although in aggregate, BMI-PRS were significantly associated with BMI, accounting for up to $1.46 \%$ of the variance. Results suggest partially shared genetic risk between MD and BMI. Genetic variation associated with BMI may only partially overlap between European and Han Chinese populations, highlighting the importance of studying genetic contributions to complex traits in diverse populations.

Grant Support: NIH K01MH113848, NARSAD Grant 28632 P\&S Fund

Schizophrenia genetic risk shared with cortical surface area but not cortical thickness: a multiplex, extended pedigree study

M.F. Pogue-Geile ${ }^{1,4}$, S.S. Kuo ${ }^{1,2}$, D.R. Roalf ${ }^{3}$, K. Prasad ${ }^{4}$, C. Musket $^{1}$, P.E. Rupert ${ }^{1}$, J. Wood ${ }^{4}$, R.C. Gur. ${ }^{3}$, L.A. Almasy ${ }^{5}$, R.E. Gur ${ }^{3}$, \& V.L. Nimgaonkar ${ }^{4}$

${ }^{1}$ Department of Psychology, University of Pittsburgh, Pittsburgh, PA, USA;

${ }^{2}$ Department of Psychiatry, UCLA, Los Angeles, CA, USA;

${ }^{3}$ Department of Psychiatry, University of Pennsylvania, Philadelphia, PA, USA;

${ }^{4}$ Department of Psychiatry, University of Pittsburgh, Pittsburgh, PA, USA;

${ }^{5}$ Department of Genetics, University of Pennsylvania, Philadelphia, PA, USA 


\section{Keywords Schizophrenia, MRI, Extended Pedigree, Genetics}

Despite schizophrenia's high heritability $\left(\mathrm{h}^{2}=.80\right)$ and patients' regionally reduced cortical volumes, the effect of its genetic risk on the cerebral cortex is poorly understood. To address this question, we employed a multiplex (two schizophrenia probands per family), extended pedigree (first to fourth degree relatives of probands) design that allows the estimation of genetic correlations between schizophrenia and cortical measures in one of the largest such studies to date. A total of 506 participants provided satisfactory MRI scans, consisting of 230 relatives ( 30 with schizophrenia) from 32 multiplex, extended pedigrees and 276 unrelated controls. Cortical thickness and surface area were estimated using Free-Surfer for 34 bilateral cortical regions. Quantitative genetic analyses using the SOLAR program with False Discovery Rate correction found that thickness and surface area for all cortical regions were significantly heritable. Although nine regions showed significantly lower cortical thickness in schizophrenia, no regions were significantly genetically correlated with schizophrenia. In contrast, 18 regions had significantly lower surface area in schizophrenia patients and seven regions were significantly genetically correlated with schizophrenia-four of which were in the frontal lobe. These results indicate that schizophrenia genetic risk primarily affects the cortex's surface area, not thickness, and primarily in regions of the frontal lobe, arguing for a focus on specific gene variants relevant to these phenotypes.

Grant Support: NIMH collaborative R01s, MH42191 (REG), MH63480 (VLN); and MH60722 (LA), T32 GM81760-10 (CWM), and CIHR Doctoral Foreign Study Award (SSK)

\section{Social disparities in childrens' and adolescents' epigenome-wide methylation profiles}

\author{
Laurel Raffington $^{1,2}$, Elliot Tucker-Drob ${ }^{1,2}$, K. Paige Harden ${ }^{1,2}$ \\ ${ }^{1}$ Department of Psychology, University of Texas at Austin, Austin, \\ TX, USA. \\ ${ }^{2}$ Population Research Center, The University of Texas at Austin, \\ Austin, TX, USA
}

Keywords Social Inequality, Social Determinants of Health, DNA Methylation, Epigenetic Clock

Children exposed to socioeconomic adversity are at prospective risk for of a variety of adult health disorders, steeper cognitive decline, and earlier mortality. The health-related antecedents of adult morbidity and mortality, such as obesity, are themselves socioeconomically graded in childhood. These findings raise two key challenging questions: (1) How does childhood adversity "get under the skin" to affect the precursors of adult disease vulnerability? and (2) What biomarkers can be useful in evaluating interventions aimed at mitigating the biological embedding of adversity? In this talk I present research conducted within the Texas Twin Project that examines epigenome-wide methylation profiles as candidate biomarkers of social health disparities. Our findings suggest promising avenues for research integrating epigenome-wide methylation profiles, polygenic scores, and family designs in advancing our understanding of human development in unequal societies.

Grant Support: We gratefully acknowledge all participants of the Texas Twin Project. This research was supported by NIH grant R01HD083613 and R01HD092548. LR is supported by the German Research Foundation (DFG). KPH and EMTD are Faculty Research Associates of the Population Research Center at the University of Texas at Austin, which is supported by a grant, 5-R24-HD042849, from the Eunice Kennedy Shriver National Institute of Child Health and Human Development (NICHD). KPH, EMTD are also supported by Jacobs Foundation Research Fellowships.

\section{Polygenic prediction of school performance in individuals with and without psychiatric disorders}

Veera M. Rajagopal ${ }^{1,2,3,4}$, Betina B. Trajberg ${ }^{2,5}$, Jakob Grove ${ }^{1,2,3,4}$, Henriette T. Horsdal ${ }^{2,5}$, Liselotte Petersen ${ }^{2,5}$, Cynthia M. Bulik ${ }^{6,7}$, iPSYCH-Broad consortium, Jonas Bybjerg-Grauholm ${ }^{2,8}$, Marie Bækvad-Hansen $^{2,8}$, David M. Hougaard ${ }^{2,8}$, Ole Mors ${ }^{2,9}$, Merete Nordentoft $^{10,11}$, Thomas Werge ${ }^{2,11,12,13}$, Preben Bo Mortensen ${ }^{2,5}$, Esben Agerbo $^{2,5}$, Anders D. Børglum ${ }^{1,2,3,4}$, Ditte Demontis ${ }^{1,2,3,4}$

${ }^{1}$ Department of Biomedicine, Aarhus University, Aarhus, Denmark;

${ }^{2}$ The Lundbeck Foundation Initiative for Integrative Psychiatric Research, iPSYCH, Denmark;

${ }^{3}$ Center for Genome Analysis and Personalized Medicine, Aarhus, Denmark;

${ }^{4}$ Centre for Integrative Sequencing, iSEQ, Aarhus University, Aarhus, Denmark;

${ }^{5}$ National Centre for Register-based Research (NCRR), Aarhus University, Aarhus, Denmark;

${ }^{6}$ Department of Psychiatry, University of North Carolina at Chapel Hill, Chapel Hill, USA;

${ }^{7}$ Department of Medical Epidemiology and Biostatistics, Karolinska Institutet, Stockholm, Sweden;

${ }^{8}$ Department for Congenital Disorders, Statens Serum Institut, Copenhagen, Denmark;

${ }^{9}$ Psychosis Research Unit, Aarhus University Hospital - Psychiatry, Aarhus, Denmark;

${ }^{10}$ Mental Health Center Copenhagen, Mental Health Services in The Capital Region of Denmark;

${ }^{11}$ Dept. Clinical Medicine, Faculty of Health Science, University of Copenhagen;

${ }^{12}$ Institute of Biological Psychiatry, Mental Health Services of Copenhagen, Copenhagen, DK;

${ }^{13}$ Center for GeoGenetics, GLOBE Institute, University of Copenhagen, Denmark

Keywords Polygenic Score; Educational Attainment; ADHD; ASD; Genetic Nurture

Individuals with psychiatric disorders often perform poorly in school compared to the general population. Both genetic and environmental factors contribute to this poor performance. Existing studies on the association of educational attainment polygenic score (EA-PGS) with school performance were based on individuals from the general population and it is unclear if the results can be generalized to individuals with psychiatric disorders. We studied the association of EAPGS with school performance in 30,982 individuals from iPSYCH; $60 \%$ of our study individuals were diagnosed with at least one major psychiatric disorder such as attention deficit hyperactivity disorder (ADHD) and autism spectrum disorder (ASD). The variance explained by the EA-PGS in school performance varied between individuals with and without psychiatric disorders. Importantly, the variances explained in school performance in those with ADHD and ASD were remarkably lower compared to that in general population. Further analysis confirmed that the observed differences were not driven by differences in socio-economic factors such as parents' education or employment status. Given that one third of the association between EA-PGS and school performance is driven by genetic nurture, our results suggest that individuals with ADHD and ASD may not be as much receptive to parental nurturing as individuals without these disorders are. Parent-offspring trios-based studies in the future may help to verify our hypothesis. 
Grant Support: Lundbeck Foundation and Klarman Family Foundation

\section{Anxiety-specific influences on substance use: evidence of a protective effect in adolescence and a risk factor in adulthood}

Maya M. Rieselbach ${ }^{1,2}$, Robin P. Corley ${ }^{2}$, John K. Hewitt ${ }^{1,2}$, and Soo Hyun Rhee ${ }^{1,2}$

${ }^{1}$ Department of Psychology and Neuroscience, University of Colorado Boulder, Boulder, CO, USA

${ }^{2}$ Institute for Behavioral Genetics, University of Colorado Boulder, Boulder, CO, USA

Keywords Internalizing, Externalizing, Anxiety, Substance Use

Externalizing behavior is a strong risk factor for substance use, but the role of internalizing manifestations of distress, and anxiety in particular, in predicting substance use remains unclear. Studies have suggested that anxiety may be either a protective or risk factor for substance use. The present study aimed to clarify whether there is an anxiety-specific pathway to substance use, examining sex and age as potential moderators that may explain the conflicting results in the literature. Anxiety was differently associated with substance use in adolescents and adults and in girls/women and boys/men. After controlling for externalizing behavior and depression, anxiety was associated with reduced substance use in adolescent girls and increased substance use in adult women. In contrast, anxiety-specific influences on substance use were not significant in boys and men. Possible explanations for these contrasting results across development (such as harm avoidance and social isolation accompanying anxiety limiting access to substances in adolescence) and sex (such as lower levels of anxiety in boys/men than in girls/women) are discussed.

Grant Support: National Institutes of Health Grants AG046938, DA011015, DA038504, and DA017637.

\section{No significant interaction between rs16969968 and genome-wide loci to predict cigarettes per day in the UK Biobank}

Pamela N. Romero Villela ${ }^{1,2^{*}}$, Richard Border, Ph.D. ${ }^{1,2}$, Matthew C. Keller, Ph.D. ${ }^{1,2}$, Luke M. Evans, Ph.D. ${ }^{1,4}$, Marissa A. Ehringer, Ph.D. ${ }^{1,3}$

${ }^{1}$ Institute for Behavioral Genetics, University of Colorado, Boulder, Colorado, USA ${ }^{2}$

${ }^{2}$ Department of Psychology, University of Colorado, Boulder, Colorado

${ }^{3}$ Department of Integrative Physiology, University of Colorado, Boulder, Colorado

${ }^{4}$ Department of Ecology \& Evolutionary Biology, University of Colorado, Boulder, Colorado

Keywords rs16969968, Nicotine, GxG, Smoking

Genes coding for the neuronal nicotinic acetylcholine receptor genes emerged as the top replicable associations with smoking behaviors from GWAS a little over a decade ago. In particular, a non-synonymous single nucleotide polymorphism (SNP) in CHRNA5, rs16969968, leads to an amino acid change (D398N) which affects the pharmacological effects of the receptor. The goal of this analysis is to determine whether we can identify other variants/genes that may interact with SNP rs16969968. Using the UK Biobank, we used
PLINK to perform a genome-wide gene-by-gene interaction analysis for cigarettes per day. We used raw $(\mathrm{M}=18.22, \mathrm{SD}=10.16)$ and $\log$ transformed $(\mathrm{M}=1.20, \mathrm{SD}=0.25)$ cigarette per day scores from 116,257 unrelated current and former smokers. Our preliminary results found no significant interaction effects between rs16969968 and genome-wide SNPs with this phenotype. It is possible that even with a large sample size such as UK Biobank, the power to detect $\mathrm{GxG}$ interactions at the genome-wide level is limited. Future exploratory analyses to examine specific subsets of genes (e.g. those that are known to be involved in nicotinic receptor biology) are in progress.

\section{Genetic variation in cognitive flexibility brain activation in schizophrenia: a multiplex extended pedigree study}

Petra E. Rupert ${ }^{1}$, David R. Roalf ${ }^{2}$, Konasale Prasad ${ }^{3}$, Susan Kuo ${ }^{1,4}$, Christie W. Musket ${ }^{1}$, Joel Wood ${ }^{3}$, Ruben C. Gur ${ }^{2}$, Laura Almasy ${ }^{5}$, Raquel E. Gur ${ }^{2}$, Vishwajit L. Nimgaonkar ${ }^{3}$, \& Michael F. PogueGeile ${ }^{1,3}$

${ }^{1}$ Department of Psychology, University of Pittsburgh, Pittsburgh, PA, USA;

${ }^{2}$ Department of Psychiatry, University of Pennsylvania, Philadelphia, PA, USA;

${ }^{3}$ Department of Psychiatry, University of Pittsburgh, Pittsburgh, PA, USA;

${ }^{4}$ Department of Psychiatry, University of California Los Angeles, Los Angeles, CA, USA;

${ }^{5}$ Department of Genetics, University of Pennsylvania, Philadelphia, PA, USA

Keywords Schizophrenia; fMRI; Cognitive Flexibility; Genetic Correlation

On executive tasks of cognitive flexibility, individuals with schizophrenia have poorer performance and often differing patterns of brain activation. The present study sought to compare the degree to which cognitive flexibility performance and its related brain activation may reflect effects of schizophrenia genetic risk using an extended pedigree design. A total of 521 participants, 30 schizophrenia probands, 202 of their relatives (1st to 4th degree), and 289 unrelated controls completed similar versions of a computerized cognitive flexibility task (Penn Conditional Exclusion Test) both out of and in an MRI scanner. Both behavioral performances and brain activation during the task in five ROIs were analyzed. In order to examine diagnostic specificity, we also investigated genetic correlations between diagnosed depression and PCET performance and brain activation. Cognitive flexibility performance was significantly genetically correlated with schizophrenia both out of $\left(R_{g}=-0.65, p\right.$ $=0.005)$ and in the scanner $\left(\mathrm{R}_{\mathrm{g}}=-0.56, P<0.001\right)$ after false discovery rate (FDR) correction. In contrast, genetic correlations between schizophrenia and ROI brain activation in the Frontal Pole (right $\operatorname{Rg}=0.30, \mathrm{p}=0.30$, left $\mathrm{Rg}=1.00, \mathrm{p}=0.01$ ), Anterior Cingulate Gyrus (bilateral $\mathrm{Rg}=0.39, \mathrm{p}=0.18$ ), and Middle Frontal Gyrus (right $\operatorname{Rg}=1.00, \mathrm{p}=0.04$, left $\operatorname{Rg}=0.60, \mathrm{p}=0.12$ ) were either not nominally significant or were not significant after FDR correction. Neither behavioral performance or brain activation measures were significantly genetically correlated with depression. In contrast to some hypotheses, these results suggest that behavioral performance on this measure of cognitive flexibility (PCET) is more sensitive (and also more specific compared with depression) to schizophrenia genetic risk effects than fMRI measures of its regional brain activation.

Grant Support: NIMH collaborative R01s MH42191 (R.E.G.), MH63480 (V.L.N.), and MH60722 (L.A.) 


\section{MAO-A as a candidate gene and childhood parental alcohol use predicting risky behaviors}

\author{
Katie Sakel ${ }^{1}$ \\ ${ }^{1}$ Departments of Biology \& Psychology, Bowling Green State \\ University, Bowling Green, Ohio, USA
}

\section{Keywords Aggression, Alcohol, Smoking, Gender}

The MAO-A gene has had previous success in candidate gene studies linking maltreatment to antisocial behavior (Baum 2003), including but not limited to conduct disorder in children (Caspl 2002, Foley 2004), aggression in psychiatric patients (Huang 2004), and alcohol use disorders in a Native American population sample (Ducci 2007). Each of these previous studies hypothesize that a childhood adversity, in the previous case maltreatment, leads to the antisocial behavior. In the present study, it is hypothesized that another adversity may be parent excessive alcohol use. Using The National Longitudinal Study of Adolescent to Adult Health dataset, five significant hierarchal regressions were completed on the following variables: (1) Participant Age to First Drink; (2) Participant Alcohol Use; (3) Participant Aggression; (4) Participant Cigarette Use; and (5) Participant Age to First Touched Sexually. The first step of the regression included the covariates of participant's birth year, sex, race, and IQ equivalent based on the grades received in high school. The second step included the number of MAO-A base pairs of each allele. The third and final step of each regression was Parent Alcohol Use. The Parent Alcohol Use only served as a predictor in Regression 1. The MAO-A gene did not predict any of the five regressions. However, differences between the steps of the covariates and between the regressions should be studied further, including why gender no longer predicts Regressions 1,3-5 after the addition of the MAO-A gene to the hierarchal regressions.

Grant Support: National Center for Family \& Marriage Research at Bowling Green State University

\section{Novel approach for parallelizing pairwise comparison problems as applied to detecting segments identical by decent in UK BioBank}

\author{
Emmanuel Sapin, Matt Keller \\ Institute for Behavioral Genetics at University of Colorado \\ Boulder
}

Keywords IBD segments, UK BioBank, Germline, Parallelization,

Pairwise comparison problems arise in many areas of science. In genomic with datasets already large and getting larger operations that require pairwise comparisons-either on pairs of SNPs or pairs of individuals-will be extremely computationally challenging. We propose a generic algorithm for pairwise comparison problems based on balanced incomplete block design and able to break a large pairwise comparison problem (of order $\mathrm{n} 2$ comparisons) into multiple smaller pairwise comparisons problems (each of order $\mathrm{n}$ comparisons). We demonstrated that this procedure is very efficient for calling IBD segments using GERMLINE in the large UK Biobank dataset, with a user time savings roughly 180-fold overrunning GERMLINE on the entire sample in our particular instance.

\section{Developmental pathways connecting polygenic risk of smoking initiation to adolescent and young-adult substance use}

Jonathan D. Schaefer, Ph.D., ${ }^{1}$ Seon-kyeong Jang, M.A., ${ }^{2}$ Mengzhen Liu, Ph.D, ${ }^{2}$ Scott Vrieze, Ph.D., ${ }^{2}$ William G. Iacono, Ph.D., ${ }^{2}$ Matt McGue, Ph.D., ${ }^{2}$ \& Sylia Wilson, Ph.D. ${ }^{1}$

${ }^{1}$ Institute for Child Development, University of Minnesota, Minneapolis, MN, USA

${ }^{2}$ Department of Psychology, University of Minnesota, Minneapolis, MN, USA

Keywords Behavioral Disinhibition, Substance Use, Polygenic Risk, Nicotine; Longitudinal

A recent genome-wide association study identified genetic variants associated with regular tobacco smoking. However, the developmental pathways through which this polygenic liability is manifested are unclear. Drawing on research that identifies risk factors for problematic substance use, we tested whether associations between polygenic risk of smoking initiation and substance use were mediated by (a) behavioral disinhibition, (b) negative emotionality, (c) cognitive ability, or (d) family socioeconomic status (SES).

We analyzed data from two longitudinal twin studies $(\mathrm{N}=2054)$ in which participants provided molecular genetic data and completed repeated, prospective measures of nicotine, cannabis, and alcohol use from early adolescence into young adulthood (ages 11 to 24 years). Multi-informant measures of behavioral disinhibition and negative emotionality, WISC-R IQ scores, and multiple indicators of family SES were collected at age 11 , when all participants were substancenaïve. We used twin participants' molecular genetic data to calculate a genome-wide polygenic score for smoking initiation and tested whether it was associated with each putative mediator assessed at age 11. We further tested whether these individual and family characteristics mediated associations between polygenic risk and subsequent cumulative substance use in later adolescence and young adulthood using structural equation modeling (SEM).

Higher polygenic risk of smoking initiation was associated with greater use of nicotine, cannabis, and alcohol in adolescence and young-adulthood (ages 14 to 24 years). Age-11 behavioral disinhibition, IQ, and family SES all emerged as partial mediators of this effect; however, SEM mediation paths through IQ and family SES were modest, significant only for nicotine use, and were reduced to non-significance in models that also included mediation paths through disinhibition. In contrast, behavioral disinhibition mediated associations between polygenic risk and cumulative use of each individual substance as well as a broader latent factor capturing general propensity to substance use, even controlling for the effects of other putative mediators.

Polygenic risk of smoking initiation captures more than simply genetic propensity towards nicotine use. In addition to predicting a broader substance-use phenotype, these scores also capture variation in early-adolescent behavioral disinhibition, and-to a lesser extentcognitive ability and aspects of the family environment. Our results suggest the testable hypothesis that helping children to increase their self-regulatory capacity may help to partly mitigate genetic risk.

Grant Support: Research reported in this article was supported by the National Institute on Drug Abuse, the National Institute on Alcohol Abuse and Alcoholism, and the National Human Genome Research Institute of the National Institutes of Health under award numbers R01DA037904 (S. V.), R01DA044283 (S. V.), R01HG008983 (S. V.), R37DA005147 (W. G. I), R01DA036216 (W. G. I.), R01DA042755 (S. V. and M. M.), R21AA026632 (S. W.), and K01DA037280 (S. W.). 
The epigenome as a biological pathway of the effects of prenatal exposure to tobacco, alcohol, and caffeine on childhood internalising problems in offspring: two large multi-cohort epigenome-wide association studies.

Laura Schellhas ${ }^{1}$, Mannan Lou $^{2}$, Giulietta Monasso ${ }^{2}$, Sílvia Fernández Barrés ${ }^{3}$, Giancarlo Pesce ${ }^{4}$, Isabella Annesi Maesano ${ }^{4}$, Christian Page $^{5}$, Stephanie London ${ }^{6}$, Charlotte Cecil ${ }^{2}$, Janine Felix ${ }^{2}$, Marcus Munafò $^{1}$, Luisa Zuccolo ${ }^{7}$, Alexandra Havdahl ${ }^{5}$, Gemma Sharp ${ }^{7}$

${ }^{1}$ Tobacco and Alcohol Research Group, School of Psychological Sciences and MRC Integrative Epidemiology Unit, University of Bristol, Bristol, UK

${ }^{2}$ Erasmus MC, University Medical Center Rotterdam, Rotterdam, the Netherlands

${ }^{3}$ Barcelona Institute for Global Health, Barcelona, Spain

${ }^{4}$ Sorbonne Université and INSERM, Paris, France

${ }^{5}$ Norwegian Institute of Public Health, Oslo, Norway

${ }^{6}$ National Institute of Environmental Health Sciences, National Institutes of Health, Department of Health and Human Services, North Carolina, USA

${ }^{7}$ MRC Integrative Epidemiology Unit, University of Bristol, Bristol, $\mathrm{UK}$

Keywords Epigenetics, Intrauterine, Smoking, Caffeine, Alcohol, Internalising Problems

Studies have found potentially causal associations between maternal health behaviours during pregnancy and offspring mental health. We explored whether this could be due to DNA methylation changes in cord blood.

We conducted two large multi-cohort epigenome-wide association studies (EWAS) of cord blood DNA methylation in relation to: (1) maternal caffeine consumption during pregnancy $(5$ cohorts; $\mathrm{n}=$ 3742); (2) childhood internalising problems ( 2 cohorts; $n=1601)$. We explored whether results from our internalising problems EWAS were enriched for $\mathrm{CpGs}$ associated with prenatal maternal caffeine exposure. We also explored enrichment for cord blood CpGs previously identified as associated with prenatal maternal smoking (Joubert 2016, $\mathrm{n}=6685$ ) and alcohol drinking (Sharp 2018, $\mathrm{n}=3075$ ).

At FDR-corrected $P<0.05$, we found $2 \mathrm{CpGs}$ associated with prenatal maternal caffeine, but no $\mathrm{CpGs}$ associated with internalising problems. At the top $5000 \mathrm{CpGs}$ with the lowest P-values in each of the maternal behaviour EWASs, 149 caffeine-associated CpGs, 139 smoking-associated CpGs and 137 alcohol-associated CpGs were associated with internalising problems with the same direction of effect and $P<0.05$. Visual inspection of QQ plots showed that $\mathrm{P}$-values in the internalising problems EWAS were inflated at the top 5000 smoking-associated CpGs, but not at the top 5000 CpGs associated with prenatal alcohol or caffeine.

In a large, multi-cohort epigenetic analysis, we found some preliminary evidence that maternal smoking could affect childhood internalising symptoms through DNA methylation, but little evidence that this is the case for prenatal alcohol and caffeine consumption.

\section{References}

Joubert, B. R., Håberg, S. E., Nilsen, R. M., Wang, X., Vollset, S. E., Murphy, S. K., Huang, Z., Hoyo, C., Midttun, Ø., Cupul-Uicab, L. A., Ueland, P. M., Wu, M. C., Nystad, W., Bell, D. A., Peddada, S. D., \& London, S. J. (2012). 450K Epigenome-Wide Scan Identifies Differential DNA Methylation in Newborns Related to Maternal Smoking during Pregnancy. Environmental Health Perspectives, 120(10), 1425-1431. https://doi.org/10.1289/ehp.1205412

Sharp, G. C., Arathimos, R., Reese, S. E., Page, C. M., Felix, J., Küpers, L. K., Rifas-Shiman, S. L., Liu, C., The Cohorts for Heart and Aging Research in Genomic Epidemiology plus (CHARGE +) methylation alcohol working group, Burrows, K., Zhao, S., Magnus, M. C., Duijts, L., Corpeleijn, E., DeMeo, D. L., Litonjua, A., Baccarelli, A., Hivert, M.-F., Oken, E., ... Zuccolo, L. (2018). Maternal alcohol consumption and offspring DNA methylation: Findings from six general population-based birth cohorts. Epigenomics, 10(1), 27-42. https://doi.org/10.2217/epi-2017-0095

Grant Support: This research was also conducted as part of the CAPICE (Childhood and Adolescence Psychopathology: unravelling the complex etiology by a large Interdisciplinary Collaboration in Europe) project, funded by the European Union's Horizon 2020 research and innovation programme, Marie Sklodowska Curie Actions - MSCA-ITN-2016 - Innovative Training Networks under grant agreement number 721567 . This study was supported by the NIHR Biomedical Research Centre at the University Hospitals Bristol NHS Foundation Trust and the University of Bristol. The views expressed in this publication are those of the authors and not necessarily those of the NHS, the National Institute for Health Research or the Department of Health and Social Care

\section{Distinct association profiles between polygenic risk for psychiatric disorder and social skill sets in the general population}

Fenja Schlag ${ }^{1}$, Marjolein van Donkelaar ${ }^{1}$, Jan Buitelaar ${ }^{2,3,4}$, Ellen Verhoef $^{1}$, Chin Yang Shapland ${ }^{1,5,6}$, Simon E. Fisher ${ }^{1,2}$, Beate St Pourcain ${ }^{1,2,5}$

${ }^{1}$ Language and Genetics, MPI for Psycholinguistics, Nijmegen, The Netherlands

${ }^{2}$ Donders Institute for Brain, Cognition and Behaviour, Radboud University, Nijmegen, The Netherlands

${ }^{3}$ Karakter Child and Adolescent Psychiatry University Centre, Nijmegen, The Netherlands

${ }^{4}$ Department of Cognitive Neuroscience, Radboud University Medical Center, Nijmegen, The Netherlands

${ }^{5}$ MRC Integrative Epidemiology Unit, University of Bristol, Bristol, UK

${ }^{6}$ Population Health Sciences, University of Bristol, Bristol, UK

Keywords Polygenic Scoring, Neurodevelopmental Disorder, Social Behaviour

Many complex, heritable neurodevelopmental disorders are linked to a spectrum of social behavioural problems. These social difficulties are thought to lie at the extreme negative end of a behavioural continuum that is shared with social traits in the general population, as supported by genetic studies. However, social behaviour is highly complex and requires multiple skills that change at different developmental stages and in different social environments. Here, we investigate associations between polygenic risk scores (PRS) of five psychiatric disorders and a spectrum of social behaviour scores in the general population. Specifically, we study associations between PRS for Attention-Deficit/Hyperactivity Disorder (ADHD), Autism Spectrum Disorders (ASD), Bipolar Disorder (BP), Major Depressive Disorder (MDD) and Schizophrenia, as informed by genome-wide summary statistics from large consortia (Cross-Disorder Group of the Psychiatric Genomics Consortium 2019), and longitudinally assessed parent- and teacher-reports for (i) low prosociality and (ii) peer problems in participants of the from the Avon Longitudinal Study of Parents And Children cohort (4-17 years, $\mathrm{N} \leq 6174$ ) (Boyd et al. 2013). For each psychiatric disorder, we assessed the heterogeneity in PRS effects using a random effects meta-regression. Our findings provide evidence for shared genetic liability between social behaviour and ADHD, ASD, MDD, and Schizophrenia, but not BP. PRS effects 
were developmentally stable, but varied (except for ASD) across reporters and, most notably, social trait; ADHD and MDD were strongly linked with peer problems, while Schizophrenia was solely associated with low prosociality. These findings suggest distinct clusters of genetic overlap between psychiatric disorder and complex social behavioural characteristics.

\section{References}

Boyd, A., Golding, J., Macleod, J., Lawlor, D. A., Fraser, A., Henderson, J., Molloy, L., Ness, A., Ring, S., \& Davey Smith, G. (2013). Cohort Profile: The 'children of the 90s'-The index offspring of the Avon Longitudinal Study of Parents and Children. Int $J$ Epidemiol, 42(1), 111-127. https://doi.org/10.1093/ije/dys064

Cross-Disorder Group of the Psychiatric Genomics Consortium. (2019). Genomic Relationships, Novel Loci, and Pleiotropic Mechanisms across Eight Psychiatric Disorders. Cell, 179(7), 1469-1482.e11. https://doi.org/10.1016/j.cell.2019.11.020

\section{Time-varying effects of genetic and parental risks for intergenerational transmission of depression on child anxiety and depressive symptoms}

Danielle M. Seay ${ }^{1}$, Jenae M. Neiderhiser ${ }^{1}$, Leslie D. Leve ${ }^{2}$, Daniel S. Shaw $^{3}$, David Reiss ${ }^{4}$, Jody M. Ganiban ${ }^{5}$, Misaki N. Natsuaki ${ }^{6}$

${ }^{1}$ Department of Psychology, The Pennsylvania State University, University Park, Pennsylvania, USA

${ }^{2}$ Department of Counseling Psychology and Human Services, University of Oregon, Eugene, Oregon, USA

${ }^{3}$ Department of Psychology, University of Pittsburgh, Pittsburgh, Pennsylvania, USA

${ }^{4}$ Yale Child Study Center, Yale School of Medicine, New Haven, Connecticut, USA

${ }^{5}$ Departments of Clinical and Developmental Psychology, George Washington University, District of Columbia, USA

${ }^{6}$ Department of Psychology, The University of California, Riverside, Riverside, California, USA

Keywords Adoption Design, Depressive/Anxiety Symptoms, Children, Time-Varying Effects, Intergenerational Transmission

Both genetic and environmental influences are important for the transmission of depression across generations. There are still key questions regarding whether these genetic and environmental influences on child symptoms are stable or variable over time, and whether there are sensitive periods during childhood in which offspring are particularly vulnerable to parents' depressive symptoms, possibly due to genetic vulnerability. To address these gaps, we examined the timevarying effects of adoptive mothers' and fathers' depressive symptoms on child anxiety/depressive symptoms and investigated moderation by genetic susceptibility for psychopathology. Participants were drawn from the Early Growth and Development Study, a longitudinal study of adopted children and their adoptive and biological parents $(\mathrm{N}=561)$. Data come from assessments that occurred when children were between 9 and 84 months. Genetic effects were low and stable during early childhood and began to increase at 54 months. Whereas adoptive mother effects increased from 13 to 54 months and then began to decrease, adoptive father effects decreased from 13 to 54 months and then began to increase. Regarding the geneenvironment interplay of influences, after 78 months, adoptive mother depressive symptoms were positively related to child symptoms among children with average and low genetic susceptibility. Similar results were found for adoptive father effects from 72 to 81 months. The current study provides a useful illustration of how time-varying effects modeling can be used to examine how genetic and familial interplay influences on child adjustment can change across developmental periods, which could, in turn, inform the development of more targeted interventions.

Grant Support: R01HD042608 R01DA020585 R01 MH092118 UG3 \& UH3 OD023389

\section{Neighborhood structural characteristics and social processes moderate the etiology of children's social problems}

Elizabeth A. Shewark ${ }^{1}$, D. Angus Clark ${ }^{2}$, Alexandra Y. Vazquez ${ }^{1}$, Kelly L. Klump ${ }^{1}$, \& S. Alexandra Burt ${ }^{1}$

${ }^{1}$ Department of Psychology, Michigan State University, East Lansing, Michigan, USA

${ }^{2}$ Department of Psychiatry, University of Michigan, Ann Arbor, Michigan, USA

Keywords Child Social Problems, Neighborhood Social Processes, Neighborhood Structural Characteristics

Neighborhood is a key context for children's social competence. Researchers have emphasized the need to consider neighborhood social processes (e.g., community monitoring and social cohesion) alongside the more standard examinations of neighborhood structural characteristics (e.g., poverty and community resources). However, studies have overlooked children's individual-level characteristics. As a consequence, little progress has been made in identifying the potential etiologic mechanisms through which neighborhood influences children's social competence. We examined a sample of 6-11 year old twin pairs that were oversampled for disadvantage $(\mathrm{N}=$ 1030), evaluating social cohesion, informal social control, available resources, and extent of problems as etiologic moderators of children's social competence. We also tested various definitions of neighborhood, including all neighbor reports within $1 \mathrm{~km}, 5 \mathrm{~km}$, and $10 \mathrm{~km}$ of the twin family homes and within census boundaries. We found that, across most definitions of neighborhood, both neighborhood social processes (informal social control and social cohesion) moderated genetic influences on children's social problems, such that as informal social control or social cohesion increased, the amount of variance accounted for by genetic factors in social problems decreased. We also examined the structural characteristics of the neighborhood. We found that extent of problems within census boundaries moderated genetic influences on social problems, such that as problems increased, the variance explained by genetic factors in social problems increased. The results suggest that structural characteristics and social processes may operate via the diathesis stress model in regards to children's social problems, such that genetic factors are more influential in deleterious neighborhood contexts.

Grant Support: F32 HD098780, R01-HD093334

\section{Genetic and environmental influences on externalizing symptoms and aspects of executive function in middle childhood}

Amanda M. Shrewsbury ${ }^{1}$, Gianna Rea-Sandin ${ }^{1}$, Sierra Clifford ${ }^{1}$, Leah D. Doane ${ }^{1}$, Kathryn Lemery-Chalfant ${ }^{1}$

${ }^{1}$ Department of Psychology, Arizona State University, Tempe, Arizona, USA 
Keywords Executive Function, Externalizing Symptoms, Genetics, Childhood, Twin

Research has linked deficits in executive function (EF), such as shortfalls in inhibition and cognitive flexibility, with externalizing problems in childhood (e.g., Schoemaker et al. 2013, Journal of Abnormal Child Psychology, 41, 457-471). However, quantitative genetic research examining associations between EF and externalizing problems in middle childhood is scarce. This study examined the genetic and environmental variation in externalizing symptoms and $\mathrm{EF}$ in a middle childhood sample. The sample comprised 710 twins (55.6\% White, $28.3 \%$ Hispanic, 3.9\% Black; $51.5 \%$ female; $M$ age = 8.42 years, $S D=0.68$ ) from the Arizona Twin Project (LemeryChalfant et al. 2019, Twin Research and Human Genetics, 22, 681-685). Externalizing symptoms were assessed with the Health and Behavior Questionnaire, and EF was assessed with the Conner's Continuous Performance Task (CPT; Conners 2000, Multi-Health Systems) and the Eriksen Flanker Task (Eriksen and Eriksen 1974, Perception \& Psychophysics, 16, 143-149). Univariate ACE models indicated that additive genetics $(\mathrm{A}=.57)$, shared $(\mathrm{C}=.32)$, and nonshared $(\mathrm{E}=.11)$ environmental influences explained variation in externalizing symptoms, whereas an AE model best represented CPT $(\mathrm{A}=.49, \mathrm{E}=.51)$ and Flanker $(\mathrm{A}=.48, \mathrm{E}=.52)$ data. Our findings suggest the importance of additive genetic influences for these traits. However, phenotypic correlations were low for externalizing symptoms with CPT data $(r=.14)$ and with Flanker data $(r=.145)$, resulting in errors when attempting to fit bivariate models to examine covariation. Given robust associations between EF and ADHD in the literature, future directions include fitting a univariate model for ADHD symptoms and bivariate models of EF and ADHD symptoms in this sample.

\section{Grant Support: R01HD079520 R01HD086085}

\section{Chasing environmental influences on school grades}

\author{
Alexandra Starr ${ }^{1}$, Rainer Riemann ${ }^{1}$ \\ ${ }^{1}$ Department of Psychology, Bielefeld University, Bielefeld, Germany
}

Keywords School Grades; Social Inequality; Twin Study; Gene-Environment Interplay; Twinlife

School performance in childhood and adolescence is an important indicator for social inequality and various life outcomes in adulthood. Previous research confirmed genetic as well as environmental influences on individual differences in school grades, yet little is known on what lies behind the environmental influences. The aim of this study is to identify external covariates that account for variance in school grades and to disentangle genetic and (non-)shared environmental components in the association between these seemingly "environmental" variables and school grades. The sample consists of 2101 pairs of monozygotic and dizygotic same-sex twins (aged 11 and 17) from the German TwinLife study. Multiple regression analysis showed that our measured external variables explain about $10 \%$ of variance in the grade point average (GPA) in both age groups. In order to determine genetic and environmental sources of this variance component, we applied a bivariate Cholesky decomposition. Results indicate that after correcting for parental socio-economic status the relation between external covariates and the GPA is entirely due to shared environmental effects at age 11, while the association between the same set of covariates and GPA at age 17 is due to common genetic sources. This pattern largely remains when considering the covariates individually: Effects are strongest for home environment and controlling parental involvement in both age groups and additionally for delinquent peers at age 17 . We discuss possible underlying effects of gene $\times$ environment interactions and provide implications for further research.

Grant Support: German Research Foundation (DFG)

\section{The multivariate genetic architecture of language- and literacy-related abilities}

Beate St Pourcain ${ }^{1,2,3}$, Ellen Verhoef ${ }^{1,4}$, George Davey Smith ${ }^{3,5}$, Simon, E. Fisher ${ }^{1,2}$, Bradley Verhulst ${ }^{6}$, Philip S. Dale ${ }^{7}$, Chin Yang Shapland ${ }^{1,3,5}$

${ }^{1}$ Language and Genetics Department, Max Planck Institute for Psycholinguistics, Nijmegen, The Netherlands

${ }^{2}$ Donders Institute for Brain, Cognition and Behaviour, Radboud University, Nijmegen, The Netherlands

${ }^{3}$ MRC Integrative Epidemiology Unit, University of Bristol, Bristol, UK

${ }^{4}$ International Max Planck Research School for Language Sciences, Nijmegen, The Netherlands

${ }^{5}$ Population Health Sciences, University of Bristol, Bristol, UK

${ }^{6}$ Texas A\&M University, Texas, USA

${ }^{7}$ Speech \& Hearing Sciences, University of New Mexico, Albuquerque, USA

Keywords Language, Literacy, Working Memory, Reading, GeneticRelationship-Matrix Structural Equation Modelling, ALSPAC

Many measures of language, literacy and phonological working memory genetically overlap, though our knowledge of their hierarchical genetic architecture is limited. Here, we structurally modelled multivariate genetic variances between reading fluency, spelling, phonemic awareness, oral language and non-word repetition in up to 6,453 unrelated ALSPAC children with phenotypic and genome-wide information. Multivariate genetic architectures where investigated with Genetic-relationship-matrix structural equation modelling (GSEM) using Cholesky and Independent Pathway models, and a combination of both models. The latter model structures the genetic variance as an Independent Pathway model (consisting of common and measurement-specific influences) and the residual variance according to a Cholesky decomposition. Model fit comparisons showed that the combined model fitted the data best. A single shared genetic factor explained the majority of genetic variance in non-word, word and passage reading fluency, ranging between $93 \%(\mathrm{SE}=4 \%)$ for passage reading accuracy ( 9 years) and $82 \%(\mathrm{SE}=9 \%$ ) for word reading speed (13 years). Utilizing passage reading accuracy as a proxy of reading fluency, we identified a shared genetic factor that explained genetic variance in reading $(97 \%(\mathrm{SE}=0.08))$, spelling $(91 \%(\mathrm{SE}=0.08))$, phonemic awareness $(98 \%(\mathrm{SE}=0.10))$, oral language $(44 \%(\mathrm{SE}=0.14))$ and non-word repetition $(53 \%(\mathrm{SE}=0.14))$. Factor structures were robust for different proxy measure selections. Measurement-specific genetic factor contributions to SNP- $h^{2}$ were found for oral language $(56 \%$ (SE $=0.14))$ and non-word repetition $(47 \%(\mathrm{SE}=0.14))$ and also for other reading fluency proxies, such as word reading speed at 13 years $(44 \%(\mathrm{SE}=0.14))$. Hence, multiple cognitive skills contribute to literacy and language performance involving pleiotropic influences augmented by measurement-specific genetic factors. 


\section{Cannabis use in college: genetic predispositions, peers, and activity participation}

Nathaniel S. Thomas, M.S.* ${ }^{1,2}$, Jessica E. Salvatore, Ph.D. ${ }^{1,5}$, Nathan A. Gillespie, Ph.D. ${ }^{4,5,6}$, Fazil Aliev, Ph.D. ${ }^{1,8}$, Albert J. Ksinan, Ph.D. ${ }^{7}$ Spit for Science Working Group, Danielle M. Dick, Ph.D.*1,2,3

${ }^{1}$ Department of Psychology, Virginia Commonwealth University, Box 842018, Richmond, VA 23284-2018

${ }^{2}$ College Behavioral and Emotional Health Institute, Virginia Commonwealth University, Box 843092, Richmond, VA 23284-3092

${ }^{3}$ Department of Human \& Molecular Genetics, Virginia Commonwealth University, Box 980033, Richmond, VA 23298-0033

${ }^{4}$ Department of Psychiatry, Virginia Commonwealth University, Box 980308, Richmond, VA 23219-1359

${ }^{5}$ Virginia Institute for Psychiatric and Behavioral Genetics, Box 980126, Richmond, VA 23298-0126

${ }^{6}$ Genetic Epidemiology, QIMR Berghofer Medical Research Institute, Locked Bag 2000 Royal Brisbane Hospital, QLD 4029, Brisbane, Queensland, Australia

${ }^{7}$ Department of Health Behavior and Policy, Virginia Commonwealth University, 830 E Main St., Richmond, VA 23219.

${ }^{8}$ Karabuk University, Faculty of Business, Turkey

Keywords Cannabis, College Student, Polygenic Risk Score, Geneby-Environment Interaction

Among adult college students in the US, cannabis use is common and associated with considerable negative consequences to health, cognition, and academic functioning, underscoring the importance of identifying risk and protective factors. Cannabis use is influenced by genetic factors, but genetic risk is not determinative. Accordingly, it is critical to identify environments that reduce risk among those who are at elevated genetic risk. This study examined the impact of genetic risk, various forms of social activity participation, and peer deviance on recent cannabis use. Our aim was to test whether these environments moderate the influence of genetic risk on cannabis use.

Participants were from the Spit for Science study, a longitudinal study of genetic and environmental influences on substance use and emotional health outcomes in a diverse sample of college students. Generalized estimating equations with a logit link function were used to examine main effects and two-way interactions. Results and implications of findings to be discussed

Grant Support: Spit for Science has been supported by Virginia Commonwealth University, P20 AA017828, R37AA011408, K02AA018755, P50 AA022537, and K01AA024152 from the National Institute on Alcohol Abuse and Alcoholism, and UL1RR031990 from the National Center for Research Resources and National Institutes of Health Roadmap for Medical Research.

This research was also supported by the National Institute on Drug Abuse of the National Institutes of Health under Award Number U54DA036105, the Center for Tobacco Products of the U.S. Food and Drug Administration, and the National Institute on Alcohol Abuse and Alcoholism under Award Number K01AA024152.

The content is solely the responsibility of the authors and does not necessarily represent the views of the NIH or the FDA. Data from this study are available to qualified researchers via dbGaP (phs001754). We would like to thank the Spit for Science participants for making this study a success, as well as the many University faculty, students, and staff who contributed to the design and implementation of the project.

We would like to thank the research participants and employees of 23andMe for making this work possible. We would also like to thank Peter Barr for his suggestions regarding the analytic plan of the current work.

\section{Symptom-level genetic modelling identifies novel risk loci and unravels the shared genetic architecture of anxiety and depression}

Jackson G Thorp ${ }^{1,2}$, Adrian I Campos ${ }^{2,3}$, Andrew D Grotzinger ${ }^{4}$, Zachary Gerring ${ }^{1}$, Jiyuan $\mathrm{An}^{5}$, Jue-Sheng $\mathrm{Ong}^{5}$, Wei Wang ${ }^{6}$, 23andMe Research Team ${ }^{6}$, Suyash Shringarpure ${ }^{6}$, Enda M Byrne ${ }^{7}$, Stuart MacGregor ${ }^{5}$, Nicholas G Martin ${ }^{3}$, Sarah E Medland ${ }^{8}$, Christel M Middeldorp ${ }^{9,10,11}$, Eske M Derks ${ }^{1}$

${ }^{1}$ Translational Neurogenomics, QIMR Berghofer Medical Research Institute, Brisbane, Australia

${ }^{2}$ Faculty of Medicine, University of Queensland, Brisbane, Australia ${ }^{3}$ Genetic Epidemiology, QIMR Berghofer Medical Research Institute, Brisbane, Australia

${ }^{4}$ Department of Psychology, University of Texas at Austin, Austin, United States

${ }^{5}$ Statistical Genetics, QIMR Berghofer Medical Research Institute, Brisbane, Australia

${ }^{6} 23$ andMe, Inc., Sunnyvale, United States

${ }^{7}$ Institute for Molecular Bioscience, University of Queensland, Brisbane, Australia

${ }^{8}$ Psychiatric Genetics, QIMR Berghofer Medical Research Institute, Brisbane, Australia

${ }^{9}$ Child Health Research Centre, University of Queensland, Brisbane, Australia

${ }^{10}$ Child and Youth Mental Health Service, Children's Health Queensland Hospital and Health Service, Brisbane, Australia

${ }^{11}$ Department of Biological Psychology, VU University Amsterdam, Amsterdam, The Netherlands

Keywords Depression Anxiety GWAS Symptom-level Overlap

Depression and anxiety are highly prevalent and comorbid psychiatric traits that cause considerable burden worldwide. Previous studies have revealed substantial genetic overlap between depression, anxiety, and a closely related personality trait-neuroticism. Here, we use factor analysis and genomic structural equation modelling (Genomic SEM) to investigate the genetic factor structure underlying 28 items assessing depression, anxiety and neuroticism. Symptoms of depression and anxiety loaded on two distinct, although genetically correlated factors, while neuroticism items were partitioned between them. We leveraged this factor structure to conduct multivariate genome-wide association analyses on latent factors of anxiety symptoms and depressive symptoms, using data from over 400,000 individuals in the UK Biobank. We identified 89 independent variants for the depressive factor (61 genomic loci) and 102 independent variants for the anxiety factor (73 loci). Of these variants, $72 \%$ and $78 \%$, respectively, replicated in an independent 23 and Me cohort of $\sim 1.9$ million individuals with self-reported diagnosis of depression (634,037 cases) and anxiety $(624,615$ cases). A pairwise GWAS analysis revealed substantial genetic overlap between anxiety and depression but also showed trait-specific genetic influences; e.g. genomic regions specific to depressive symptoms were associated with hypertriglyceridemia, while regions specific to anxiety symptoms were linked to blood pressure phenotypes. The substantial genetic overlap between the two traits was further evidenced by a lack of trait-specificity in polygenic prediction of depressive and anxiety symptoms. Our results provide novel insight into the genetic architecture of depression and anxiety and comorbidity between them.

Grant Support: J.G.T. and A.I.C. are supported by a UQ Research Training Scholarship from The University of Queensland (UQ). N.G.M. received funding from the Australian National Health \& Medical Research Council (NHMRC) to conduct surveys in the Australian Twin Registry. S.M. is supported by an NHMRC Fellowship. 


\section{Assortative mating and the usefulness of polygenic scores in extended pedigrees}

Fartein Ask Torvik ${ }^{1,2}$, Espen Moen Eilertsen ${ }^{3}$, Laurie Hannigan ${ }^{4}$, Alexandra Havdahl ${ }^{3,4,5}$, Eivind Ystrom ${ }^{3,5}$

${ }^{1}$ Centre for Fertility and Health, Norwegian Institute of Public Health, Oslo, Norway

${ }^{2}$ Department of Psychology, University of Oslo, Oslo, Norway

${ }^{3}$ PROMENTA Research Center, Department of Psychology, University of Oslo, Oslo, Norway

${ }^{4}$ Nic Waals Institute, Lovisenberg Diaconal Hospital, Oslo, Norway

${ }^{5}$ Department of Mental Disorders, Norwegian Institute of Public Health, Oslo, Norway

Keywords Assortative mating, polygenic scores, structural equation modelling

Partners resemble each other in many traits, including educational attainment, psychopathology, and height. Mating is often assumed to be random in genetic studies and the results can be biased when it is not. Polygenic scores offer a straightforward means of assessing the genetic similarity of partners that arises from assortative mating. However, estimating the correlation between partners' polygenic scores is likely to provide an underestimate of the actual genetic correlation because polygenic scores are imperfect measures of the genetic factors that give rise to a phenotype. Structural equation modelling (SEM), which is commonly used with data from twins and other kinds of relatives, provides a flexible way to incorporate polygenic scores with phenotypic data from multiple individuals. Incorporating polygenic scores in an SEM framework allows an estimation of the relationship between latent variables (genetic factors) that are the topic of the research, rather than the observed indicators of these (polygenic scores). We use phenotypic data on educational attainment, height, and symptoms of major depressive disorder from 70107 mother-father dyads and polygenic scores on 13096 mother-father dyads participating in the Norwegian Mother, Father, and Child Cohort Study. We present a novel structural equation model which uses data on phenotypes and polygenic scores for unrelated individuals to estimate heritability and genetic correlations-in this case, genetic correlations between partners-including results for educational attainment, psychopathology, and height.

Grant Support: Research Council of Norway (300668, 273659, 262177).

\section{Vaccine willingness and socioeconomic status: a biometrically controlled design}

Jonathan Trattner ${ }^{1}$, Kennon Later $^{2}$, S. Mason Garrison ${ }^{2}$

${ }^{1}$ Department of Interdisciplinary Studies, Wake Forest University Winston-Salem, North Carolina, USA

${ }^{2}$ Department of Psychology, Wake Forest University, Winston-Salem, North Carolina, USA

Keywords Sibling-comparison; Health Behaviors; Influenza; Vaccination; Socioeconomic Status

Recent reports detail health disparities amongst ethnic minorities during the Covid-19 pandemic (Hooper et al. 2020). These disparities are often attributed to socioeconomic status (SES), pre-existing health conditions (Ssentego et al. 2020), or Covid-19 severity (Yang et al. 2020). Interventions to mitigate these disparities must explicitly account for known gene-and-environmental confounds (Garrison and Rodgers 2019; Williams et al. 2020).
Given the oft-mentioned necessity for a Covid-19 vaccine before returning to normalcy, we aim to identify the relationship between SES and vaccination willingness through a sibling-comparison design using the influenza vaccine as a proxy. We use data from the 1979 National Longitudinal Survey of Youth $(\mathrm{n}=12,686)$. Applying a discordant-kinship model, we found a significant relationship between SES and vaccine willingness, independent of gene-and-environmental variance.

\section{References}

Hooper, Nápoles, \& Pérez-Stable (2020). COVID-19 and Racial/ Ethnic Disparities. JAMA.

Garrison \& Rodgers (2019). Decomposing the causes of the socioeconomic status-health gradient with biometrical modeling. JPSP, 116(6), 1030-1047.

Ssentongo, P., Ssentongo, A., Heilbrunn, Ba, \& Chinchilli (2020). The association of cardiovascular disease and other pre-existing comorbidities with COVID-19 mortality: A systematic review and meta-analysis. MedRxiv.

Williams, Freydin, Mangino, Couvreur, Visconti, Bowyer, Roy, Falchi, Sudre, Davies, Hammond, Menni, Steves, \& Spector. (2020). Self-reported symptoms of covid-19 including symptoms most predictive of SARS-CoV-2 infection, are heritable. MedRxiv.

Yang, Gui, \& Xiong (2020). Comparison of Clinical Characteristics of Patients with Asymptomatic vs Symptomatic Coronavirus Disease 2019 in Wuhan, China. JAMA Network Open, 3(5)

\section{The lifespan intellectual continuity project in the Louisville twin study}

Eric Turkheimer ${ }^{1}$, Evan J. Giangrande ${ }^{1}$, Christopher R. Beam ${ }^{2}$ ${ }^{1}$ Department of Psychology, University of Virginia; ${ }^{2}$ Department of Psychology, University of Southern California

Keywords Intelligence, WISC, WAIS, Twins, Lifespan

Over a span of forty years, the Louisville Twin Study (LTS) collected longitudinal intellectual ability data from twin children between the ages of three months and fifteen years, as well as from their siblings and parents. Full in person Wechsler intelligence tests were obtained starting at age seven. The data structure of the LTS is complex but rich, comprising an intersection of cohort, age, and three different versions of the WISC. Many crucial questions about the development of intellectual ability require assumptions about the measurement equivalence of ability across cohort, age and test version; to the best of our knowledge there is no existing dataset that allows all such equivalence to be modeled and tested in a single sample. In addition, the currently funded Louisville Twin Study is following up the original twins, who are now middle-aged. Issues of continuity between Wechsler adult and childhood scales are even more difficult, because the subscales do not share items. We will describe the goals and procedures of the current project, including: (1) Entering the item data from the original WISC data from the LTS; (2) Development of vertical linking models that will allow WISC scores to be measured on an invariant IRT-based scale; (3) Tracing the development of cognitive ability in childhood on an interval scale that is not agestandardized; (4) Design of additional items to be added to the WAIS providing continuity between childhood and adulthood; (5) Models of the Flynn Effect and detection of early cognitive decline in midlife.

Grant Support: National Institute on Aging Grant Nos. R03AG048850-01 and R01AG063949-01. 


\section{Continuity of polygenetic influences on aggressive behavior across the life course}

Camiel M. van der Laan ${ }^{1,2}$, José J. Morosoli-García ${ }^{3}$, Lucia ColodroConde $^{3}$, Sarah Medland ${ }^{3}$, Nicholas G. Martin ${ }^{3}$, Jaakko Kaprio ${ }^{4}$, Teemu Palviainen ${ }^{5}$, Steve G.A. van de Weijer ${ }^{2}$, Michel G. Nivard ${ }^{1}$, and Dorret I. Boomsma ${ }^{1}$

${ }^{1}$ Department of Biological Psychology, Vrije Universiteit, Amsterdam, The Netherlands

${ }^{2}$ The Netherlands Institute for the Study of Crime and Law Enforcement, Amsterdam, The Netherlands.

${ }^{3}$ QIMR Berghofer Medical Research Institute, Brisbane, Queensland, Australia.

${ }^{4}$ Institute for Molecular Medicine, Helsinki, Finland.

Keywords Aggressive behavior, Aggression, Polygenetic Scores, Life Course, Age

Aggressive behavior tends to decrease with age. However, also at higher ages there is still much variation in the extent to which individuals exhibit aggressive behavior. In most cases, individuals retain their relative positions in the extent of aggressive behavior within their age group. The most aggressive child will most likely grow up to be the most aggressive adult. In this study, we investigate whether genetic risk factors in childhood also increase risk for aggressive behavior in adolescence and adulthood.

In three genotyped cohorts from the Netherlands, Australia, and Finland, we calculated polygenic scores based on a large genome wide meta-analysis of aggression phenotypes in children (Ip et al., submitted for publication). We then modelled the effect of these polygenic scores on aggression in six age-bins with generalized equation estimation. Additionally, we modelled the interaction between the polygenetic scores and age by applying 'rolling weights' to mixed effects models, with the weights of observations shifting from age 12 to 70, in increments of one year.

Preliminary results suggest we can predict aggressive behavior in adolescents and adults from polygenic scores based on childhood aggressive behavior. The effect decreases with age, diminishing around age 35 . Results suggest that genetic factors play a role in continuity of aggression from childhood to adulthood.

References

Ip, H.F. et al. (2020). Genetic Association Study of Childhood Aggression across raters, instruments and age. Submitted for publication. https://www.biorxiv.org/content/10.1101/854927v1

\section{Multivariate GREML analysis reveals shared genetic architecture between brain regions and behavioral traits}

Ronald De Vlaming ${ }^{1}$, Eric A.W. Slob ${ }^{2,3}$, Philip Jansen ${ }^{4,5,6}$, Philipp D. Koellinger ${ }^{1}$, Patrick J.F. Groenen ${ }^{7}$, Cornelius A. Rietveld ${ }^{2,3}$

${ }^{1}$ School of Business and Economics, Vrije Universiteit Amsterdam, Amsterdam, the Netherlands

${ }^{2}$ Erasmus University Rotterdam Institute for Behavior and Biology, Erasmus University Rotterdam, Rotterdam, the Netherlands

${ }^{3}$ Department of Applied Economics, Erasmus School of Economics, Erasmus University Rotterdam, Rotterdam, The Netherlands)

${ }^{4}$ Amsterdam Neuroscience, Vrije Universiteit Amsterdam, Amsterdam, the Netherlands,

${ }^{5}$ Department of Complex Trait Genetics, Center for Neuroscience and Cognitive Research, Amsterdam Neuroscience, Vrije Universiteit Amsterdam, Amsterdam, the Netherlands
${ }^{6}$ Department of Clinical Genetics, VU Medical Center, Amsterdam UMC, Amsterdam, the Netherlands

${ }^{7}$ Econometric Institute, Erasmus School of Economics, Rotterdam, the Netherlands

\section{Keywords GREML, Brain Regions, Behavioral Traits.}

Genome-based Restricted Maximum Likelihood (GREML) estimation has been developed to estimate the SNP-heritability of traits using observed genetic similarities among unrelated individuals in a population. The bivariate extension of this method enables the estimation of the genetic correlation between two traits. One often combines the estimates of pairwise combinations of traits into a multivariate genetic correlation matrix in case one is interested in the genetic correlation across more than two traits. However, this "pairwise bivariate" approach may result in a genetic correlation matrix which is not internally consistent. Besides, the corresponding standard errors of this genetic correlation matrix are not completely correct as they do not reflect the full data structure. Therefore, we developed a multivariate extension of the GREML method which guarantees the internal consistency of the resulting genetic correlation matrix using a Cholesky decomposition. To deal with the computational complexity of the model, we developed an optimization procedure which is more efficient than the present "pairwise bivariate" approach (reducing complexity from order (NP) ${ }^{3}$ to $\mathrm{NP}^{3}$ ).

We used the multivariate GREML method to analyze the shared genetic architecture of the human brain (76 cortical and subcortical structures) and 10 behavioral traits. Using a sample of approximately 14,000 unrelated individuals from the UK Biobank, one of our main findings is that two genetically distinct clusters in the brain are associated with intelligence. Besides the empirical value of these findings, the resulting multivariate genetic correlation matrix can also be used as input for methods such as genomic structural equation modeling (Genomic-SEM).

Grant Support: This research has been conducted using the UK Biobank Resource under Application Number 11425. We would like to thank the participants and researchers from UK Biobank who significantly contributed or collected data. This work was carried out on the Dutch national e-infrastructure with the support of SURF Cooperative. P.D.K. was supported by an European Research Council consolidator Grant (647648 EdGe).

\section{Exploring genetic relationships between early motor, personal-social and language development}

Jeffrey van der Ven ${ }^{1,4}$ Ellen Verhoef ${ }^{1}$, Mitchell Olislagers ${ }^{4}$, Janne Vermeulen $^{1,4}$, Philip Dale ${ }^{5}$, Simon E. Fisher ${ }^{1,2}$, Beate St Pourcain ${ }^{1,2,3}$ ${ }^{1}$ Language and Genetics, MPI for Psycholinguistics, Nijmegen, The Netherlands

${ }^{2}$ Donders Institute for Brain, Cognition and Behaviour, Radboud University, Nijmegen, The Netherlands

${ }^{3}$ Bristol Medical School, University of Bristol, Bristol, UK

${ }^{4}$ Medical Biology, Radboud University, Nijmegen, The Netherlands ${ }^{5}$ Speech \& Hearing Sciences, University of New Mexico, Albuquerque, USA

Keywords Personal-social development, Structural equation modelling, motor/language development, genetic overlap, Early development

During the first years of life, children learn to master many developmental milestones. Specifically, the transition from crawling to walking during motor development is thought to initiate a cascade of developmental events across domains, including personal-social skills 
and language development, due to largely unknown aetiological mechanisms. Here, we study whether infant fine motor, gross motor and personal-social skills (6-18 months) genetically predict processes of language acquisition (15-38 months) in unrelated children (genetic-relatedness $<0.05)$ from the Avon Longitudinal Study of Parents and Children $(\mathrm{N}<7923,50.6 \%$ males, 15 Communicative Development Inventories (CDI) and Denver Development Screening Test (DDST) measures and genome-wide data). Univariate and multivariate genetic architectures were analyzed using Genome-wide Complex Trait Analysis and Genetic-Relationship-Matrix Structural Equation modelling (GSEM; Cholesky decomposition). Identified SNP-heritability estimates ranged from $4 \%(\mathrm{SE}=0.06)$ to $20 \%$ (SE $=$ 0.05). Both, gross motor skills at 6 months and personal-social skills at 15 months of age were genetically correlated with later language abilities (e.g. expressive vocabulary 24 months: $r_{g}=0.65(\mathrm{SE}=0.23$, $\mathrm{p}=0.007)$ and $\mathrm{r}_{\mathrm{g}}=0.61(\mathrm{SE}=0.19, \mathrm{p}=0.001)$, respectively). GSEM analyses showed that expressive vocabulary (24 months) and word combination use (38 months) load on the latent genetic factor for gross motor skills (6 months), with standardised factor loadings of $0.25(\mathrm{SE}=0.09, \mathrm{p}=0.006)$ and $0.25(\mathrm{SE}=0.09, \mathrm{p}=0.006)$ respectively. Similarly, expressive vocabulary (24 months) and grammar (24 months) loaded on the latent genetic factor for personalsocial skills (15 months) with standardised factor loadings of 0.29 (SE $=0.09, \mathrm{p}=0.001)$ and $0.26(\mathrm{SE}=0.09, \mathrm{p}=0.004)$, respectively. Thus, both infant gross motor skills and personal-social skills are predictive of subsequent language performance, consistent with developmental priming of language abilities.

\section{Dissecting genetic from environmental associations for well-being}

Margot van de Weijer ${ }^{1,2}$, Bart Baselmans ${ }^{3}$, Eveline de Zeeuw ${ }^{1,2}$, Jouke-Jan Hottenga ${ }^{1,2}$, Eivind Ystrøm ${ }^{4,5,6}$ \& Meike Bartels ${ }^{1,2}$

${ }^{1}$ Department of Biological Psychology, Faculty of Behavioural and Movement Sciences, Vrije Universiteit Amsterdam, The Netherlands ${ }^{2}$ Amsterdam Public Health Research Institute, Amsterdam University Medical Centre, Amsterdam, The Netherlands.

${ }^{3}$ Institute for Molecular Bioscience, The University of Queensland, Brisbane, QLD, Australia.

${ }^{4}$ Department of Mental Disorders, Norwegian Institute of Public Health, Oslo, Norway.

${ }^{5}$ PROMENTA Research Center, Department of Psychology, University of Oslo, Oslo, Norway.

${ }^{6}$ School of Pharmacy, University of Oslo, Oslo, Norway.

Keywords Well-Being, Genetic Nurture, MZ differences, Genetic Association.

Meta-analyses have shown that approximately $40 \%$ of individual differences in well-being can be explained by genetic factors (Bartels 2015; Nes and Røysamb 2015). This leads to questions about the extent to which environmental and genetic influences for well-being are intertwined. We present two ongoing projects where we aim to get better insight into the complex interplay between genetic and environmental factors and well-being. First, we present an ongoing project where we use three methods (extended twin-family design, nontransmitted alleles polygenic scores and M-GCTA) to examine the potential presence of genetic nurture in Netherlands Twin Register (NTR) and Norwegian Mother, Father and Child Cohort Study $(\mathrm{MoBa})$ participants. Second, we present another ongoing project where we aim to examine potential genetic components in phenotypic associations between well-being and a range of social factors. For this project, we examine monozygotic within-pair difference scores, bivariate genetic models, and within-individual difference scores across two time-points in NTR adolescents. By combining the knowledge of these two projects, we aim to achieve two important goals. First, it allows us to gain more insight into how genetic and environmental factors combine to influence individual differences in well-being. Second, these analyses provide insight in the potential bias that occurs when genetic and environmental factors are not both taken into consideration when studying well-being.

Grant Support: This work was supported by the European Research Council (ERC-COG WELL-BEING 771057, PI Bartels)

\section{DNA methylation signatures of a broad spectrum of aggressive behavior: a meta-analysis of epigenome- wide studies across the lifespan}

Jenny van Dongen ${ }^{1}$, Fiona A. Hagenbeek ${ }^{1}$, Matthew Suderman ${ }^{2,3}$, Peter Roetman ${ }^{4}$, Karen Sugden ${ }^{5,6}$, Andreas G. Chiocchetti ${ }^{7}$, Khadeeja Ismail $^{8}$, Rosa H. Mulder ${ }^{9,10,11}$, Jonathan Hafferty ${ }^{12}$, Mark J. Adams ${ }^{12}$, Rosie M. Walker ${ }^{13}$, Stewart W. Morris,BSc ${ }^{13}$, Jari Lahti ${ }^{14,15}$, Leanne K. Küpers ${ }^{16}$, Georgia Escaramis ${ }^{17,18,19}$, Silvia Alemany ${ }^{20,21,22}$, Marc Jan Bonder ${ }^{23}$, Mandy Meijer ${ }^{24}$, Hill F. Ip ${ }^{1}$, Rick Jansen ${ }^{25}$, Bart M. L. Baselmans ${ }^{1}$, Priyanka Parmar ${ }^{26,27}$, Estelle Lowry ${ }^{26,28}$, Fabian Streit $^{29}$, Lea Sirignano ${ }^{29}$, Tabea Send ${ }^{30}$, Josef Frank ${ }^{29}$, Juulia Jylhävä ${ }^{31}$, Yunzhang Wang ${ }^{31}$, Pashupati Prasad Mishra ${ }^{32}$, Olivier F. Colins $^{4,33}$, David Corcoran ${ }^{6}$, Richie Poulton ${ }^{34}$, Jonathan Mill ${ }^{35}$, Eilis J. Hannon $^{35}$, Louise Arseneault ${ }^{36}$, Tellervo Korhonen ${ }^{8}$, Eero Vuoksimaa $^{8}$, Janine Felix ${ }^{11,37}$, Marian Bakermans-Kranenburg ${ }^{38}$, Archie Campbell, MA ${ }^{13}$, Darina Czamara ${ }^{14}$, Elisabeth Binder ${ }^{14}$, Eva Corpeleijn ${ }^{16}$, Juan Ramon González ${ }^{20,21,22}$, Regina Grazuleviciene ${ }^{39}$, Kristine B. Gutzkow ${ }^{40}$, Jorunn Evandt ${ }^{40}$, Marina Vafeiadi ${ }^{41}$, Marieke Klein ${ }^{24,42}$, Dennis van der Meer ${ }^{43,44}$, Lannie Ligthart ${ }^{1}$, Cornelis Kluft $^{45}$, Gareth E. Davies ${ }^{46}$, Christian Hakulinen ${ }^{15}$, Liisa KeltikangasJärvinen $^{15}$; Barbara Franke 24,47 , Christine M. Freitag 7 , Kerstin Kon$\operatorname{rad}^{48,49}$, Amaia Hervas ${ }^{50}$ Aranzazu Fernández-Rivas ${ }^{51}$, Agnes Vetro $^{51}$, Olli Raitakari ${ }^{53,54,55}$, Terho Lehtimäki ${ }^{32}$, Robert Vermeiren $^{4,56}$, Timo Strandberg ${ }^{57}$, Katri Räikkönen ${ }^{15}$, Harold Snieder ${ }^{16}$, Stephanie H. Witt ${ }^{29}$, Michael Deuschle ${ }^{30}$, Prof, Nancy L. Pedersen ${ }^{31}$, Sara Hägg ${ }^{31}$, Jordi Sunyer ${ }^{20,21,22,58}$, Lude Franke ${ }^{23}$, Jaakko Kaprio ${ }^{8}$, Miina Ollikainen ${ }^{8}$, Terrie E. Moffitt ${ }^{5,6,36,59}$, Henning Tiemeier ${ }^{10,60}$, Marinus H. van Ijzendoorn ${ }^{61,62}$, Caroline Relton ${ }^{2,3}$, Martine Vrijheid $^{20,21,22}$, Sylvain Sebert ${ }^{26,27,63}$, Marjo-Riitta Jarvelin ${ }^{26,27,64}$, Avshalom Caspi ${ }^{5,6,36,59}$, Kathryn L. Evans ${ }^{13}$, Andrew M. McIntosh ${ }^{12}$, Meike Bartels ${ }^{1}$, Dorret Boomsma ${ }^{1}$

${ }^{1}$ Department of Biological Psychology, Amsterdam Public Health Research Institute, Vrije Universiteit Amsterdam, Amsterdam, The Netherlands

${ }^{2}$ Population Health Sciences, Bristol Medical School, University of Bristol, Bristol, UK.

${ }^{3}$ MRC Medical Research Council Integrative Epidemiology Unit, University of Bristol, Bristol, UK.

${ }^{4}$ Curium-LUMC, Department of Child and Adolescent Psychiatry, Leiden University Medical Center in Oegstgeest, The Netherlands

${ }^{5}$ Department of Psychology and Neuroscience, Duke University, Durham, NC, USA.

${ }^{6}$ Center for Genomic and Computational Biology, Duke University, Durham, NC, USA

${ }^{7}$ University Hospital Frankfurt; Department of Child and Adolescent Psychiatry, Psychosomatics and Psychotherapy; Frankfurt am Main, Germany

${ }^{8}$ Institute for Molecular Medicine Finland, University of Helsinki, Helsinki, Finland

${ }^{9}$ Institute of Education and Child Studies, Leiden University, Leiden, the Netherlands. 
${ }^{10}$ Department of Child and Adolescent Psychiatry/Psychology, Erasmus MC, University Medical Center Rotterdam, Rotterdam, the Netherlands.

${ }^{11}$ Generation R Study Group, Erasmus MC, University Medical Center Rotterdam, Rotterdam, the Netherlands

${ }^{12}$ Division of Psychiatry, University of Edinburgh

${ }^{13}$ Centre for Genomic and Experimental Medicine, Institute of Genetics and Molecular Medicine, University of Edinburgh

${ }^{14}$ Turku Institute for Advances Studies, University of Turku, Turku Finland

${ }^{15}$ Department of Psychology and logopedics, Faculty of Medicine, University of Helsinki, Helsinki, Finland

${ }^{16}$ University of Groningen, University Medical Center Groningen, Department of Epidemiology, Groningen, The Netherlands

${ }^{17}$ CIBER Epidemiología y Salud Pública (CIBERESP), Barcelona, Spain

${ }^{18}$ Dpt. of Biomedical Science, Faculty of Medicine and Health Science, University of Barcelona, Barcelona, Spain

${ }^{19}$ Research Group on Statistics, Econometrics ans Health (GRECS), UdG

${ }^{20}$ ISGlobal, Barcelona, Spain.

${ }^{21}$ Universitat Pompeu Fabra (UPF), Barcelona, Spain.

${ }^{22}$ CIBER Epidemiología y Salud Pública (CIBERESP), Barcelona, Spain

${ }^{23}$ Department of Genetics, University of Groningen, University Medical Centre Groningen, Groningen, The Netherlands

${ }^{24}$ Department of Human Genetics, Donders Institute for Brain, Cognition and Behavior, Radboud University Medical Center, Nijmegen, The Netherlands

${ }^{25}$ Department of Psychiatry, Amsterdam UMC, Vrije Universiteit Amsterdam, Amsterdam, the Netherlands

${ }^{26}$ Center for Life Course Health Research, P.O. Box 5000, 90014, University of Oulu, Oulu, Finland.

${ }^{27}$ Biocenter Oulu, University of Oulu, P.O. Box 5000, 90014, Finland

${ }^{28}$ Queen's University Belfast

${ }^{29}$ Department of Genetic Epidemiology in Psychiatry, Central Institute of Mental Health, Medical Faculty Mannheim, University of Heidelberg, Mannheim, Germany

${ }^{30}$ Department of Psychiatry and Psychotherapy, Central Institute of Mental Health, Medical Faculty Mannheim, University of Heidelberg, Germany

${ }^{31}$ The Department of Medical Epidemiology and Biostatistics, Karolinska Institutet, Nobels väg 12A, 17165 Stockholm, Sweden

${ }^{32}$ Department of Clinical Chemistry, Fimlab Laboratories, and Finnish Cardiovascular Research Center - Tampere, Faculty of Medicine and Health Technology, Tampere University, Tampere 33520, Finland

${ }^{33}$ Department of Special Needs Education, Ghent University, Ghent, Belgium

${ }^{34}$ Dunedin Multidisciplinary Health and Development Research Unit, Department of Psychology, University of Otago, Dunedin, New Zealand

${ }^{35}$ University of Exeter Medical School, University of Exeter, Exeter, UK

${ }^{36}$ Social, Genetic and Developmental Psychiatry Centre, Institute of Psychiatry, Psychology and Neuroscience, King's College London, London, UK

${ }^{37}$ Department of Pediatrics, Erasmus MC, University Medical Center Rotterdam, Rotterdam, the Netherlands

${ }^{38}$ Clinical Child \& Family Studies, Vrije Universiteit Amsterdam, Amsterdam, the Netherlands

${ }^{39}$ Department of Environmental Sciences, Vytautas Magnus University, K. Donelaicio str. 58, 44248 Kaunas, Lithuania

${ }^{40}$ Norwegian Institute of Public Health

${ }^{41}$ Department of Social Medicine, University of Crete, Greece
${ }^{42}$ University Medical Center Utrecht, UMC Utrecht Brain Center, Department of Psychiatry, Utrecht, The Netherlands

${ }^{43}$ NORMENT, Division of Mental Health and Addiction, Oslo University Hospital \& Institute of Clinical Medicine, University of Oslo, Oslo, Norway

${ }^{44}$ School of Mental Health and Neuroscience, Faculty of Health, Medicine and Life Sciences, Maastricht University, Maastricht, The Netherlands

${ }^{45}$ Good Biomarker Sciences, Leiden, the Netherlands

${ }^{46}$ Avera Institute for Human Genetics, 3720 W. 69th Street, Sioux Falls, SD, 57108, USA

${ }^{47}$ Department of Psychiatry, Donders Institute for Brain, Cognition and Behavior, Radboud University Medical Center, Nijmegen, The Netherlands

${ }^{48}$ University Hospital, RWTH Aachen, Child Neuropsychology Section, Department of Child and Adolescent Psychiatry, Psychosomatics and Psychotherapy, Aachen, Germany

${ }^{49}$ JARA-Brain Institute II, Molecular Neuroscience and Neuroimaging (INM-11), RWTH Aachen \& Research Centre Juelich, Germany ${ }^{50}$ Hospital Universitario Mutua de Terrassa, Child and Adolescent Mental Health Service, Barcelona, Spain

${ }^{51}$ Bilbao University Hospital, Psychiatric Service, Osakidetza, Bilbao, Spain

${ }^{52}$ Szeged University, Department of Pediatrics and Pediatrics health center, Child and Adolescent Psychiatry, Szeged, Hungary

${ }^{53}$ Centre for Population Health Research, University of Turku and Turku University Hospital, Turku, Finland

${ }^{54}$ Research Centre of Applied and Preventive Cardiovascular Medicine, University of Turku, Turku, Finland

${ }^{55}$ Department of Clinical Physiology and Nuclear Medicine, Turku University Hospital, Turku, Finland

${ }^{56}$ Youz, Parnassia Group, The Hague, the Netherlands

${ }^{57}$ Helsinki University Central Hospital, Geriatrics, Helsinki, Finland.

${ }^{58}$ IMIM (Hospital del Mar Medical Research Institute), Barcelona, Spain

${ }^{59}$ Department of Psychiatry and Behavioral Sciences, Duke University School of Medicine, Durham, N.C., U.S.A

${ }^{60}$ Department of Social and Behavioral Science, Harvard TH Chan School of Public Health, Boston USA

${ }^{61}$ Department of Psychology, Education and Child Studies, Erasmus University Rotterdam, Rotterdam, the Netherlands.

${ }^{62}$ School of Clinical Medicine, University of Cambridge, Cambridge, UK

${ }^{63}$ Section of Genomics of Common Disease, Department of Medicine, Imperial College. Paddington, W2 IPG, London, United Kingdom London, Hammersmith Hospital Campus, Burlington Danes Building, Du Cane Road, London, W12 0NN, UK

${ }^{64}$ MRC-PHE Centre for Environment and Health, Imperial College London, Praed Street Wing, St Mary's Campus, Paddington, W2 IPG, London, United Kingdom London, Hammersmith Hospital Campus, Burlington Danes Building, Du Cane Road, London, W12 0NN, UK

Keywords Aggression, DNA methylation, EWAS, aggressive behavior, epigenetics

Here we report an EWAS meta-analysis (EWAMA) of broadly defined aggressive behavior in peripheral blood samples for 14,434 participants from 18 cohorts with mean ages ranging from 7 to 68 years, and cord blood samples for 2,425 children from 5 cohorts with aggression assessed at ages ranging from 4 to 7 years. In an additional group of 1235 9-year old children and 172 children enrolled in a childhood psychiatric clinic, buccal cell-derived DNA methylation profiles were available.

In blood samples, 13 methylation sites were significantly associated with aggression after Bonferroni correction. No epigenome-wide significant sites were found in cord blood samples, but $83 \%$ of these 
sites showed the same direction of association in cord blood with aggression in childhood $(\mathrm{r}=0.74, \mathrm{p}=0.006)$. Regression coefficients based on analyses in buccal cells and blood overall showed no directional consistency.

Top-sites (FDR < 0.05) from the analysis of blood samples in children and adults were previously reported for association with chemical exposures, smoking, cognition, metabolic traits, and mQTLs. Three genes whose expression levels were associated with top-sites were previously linked to schizophrenia and general risk tolerance, pointing to loci that are correlated with substance use and chemical exposures, as well as genetic variation. At some loci, DNA methylation variation in blood mirrors variation in the brain.

Epigenetic profiles may capture lifetime cumulative effects of genetic, stochastic, and environmental influences associated with aggression. Our findings may serve as potential biomarkers and possibly indicate causal relationships with aggression and form a basis for future projects.

Grant Support: This work was supported by ACTION. ACTION receives funding from the European Union Seventh Framework Program (FP7/2007-2013) under grant agreement no 602768. JvD is supported by the NWO-funded X-omics project (184.034.019).

\section{Exploring the etiology of resilience to neighborhood disadvantage}

Alexandra Y. Vazquez ${ }^{1}$, Elizabeth A. Shewark ${ }^{1}$, David A. Clark ${ }^{2}$, Kelly L. Klump ${ }^{1}$, Luke W. Hyde ${ }^{3}$, and S. Alexandra Burt ${ }^{1}$

${ }^{1}$ Department of Psychology, Michigan State University, East Lansing, Michigan, USA

${ }^{2}$ Department of Psychiatry, University of Michigan, Ann Arbor, Michigan, USA

${ }^{3}$ Department of Psychology, University of Michigan, Ann Arbor, Michigan, USA

Keywords Neighborhood Disadvantage, Resilience, Twins, Common Pathway

Early-life exposure to disadvantage predicts numerous health and academic disparities. Despite this, studies demonstrate that upwards of $40-60 \%$ of youth living in disadvantaged contexts evidence resilient outcomes (Vanderbilt-Adriance and Shaw 2008). Although these youth provide a model of successful adaptation to adversity, relatively little is known about the factors enabling their positive outcomes. The current study utilizes a sample of twin pairs $(\mathrm{N}=417)$ living in disadvantaged contexts (i.e. family poverty, neighborhood poverty, community violence) to elucidate the etiology of resilience within and across domains. Three univariate ACE models were utilized to first explore the etiology of distinct domains of resilience: social resilience, academic resilience, psychiatric resilience. The $\mathrm{A}, \mathrm{C}$, and $\mathrm{E}$ variances were all statistically significant for social resilience $(\mathrm{A}=$ $22 \%, \mathrm{C}=61 \%, \mathrm{E}=17 \%)$ and psychiatric resilience $(\mathrm{A}=40 \%, \mathrm{C}=$ $28 \%, \mathrm{E}=32 \%)$, but only the $\mathrm{A}$ and $\mathrm{E}$ variances were statistically significant for academic resilience $(A=65 \%, C=0 \%, E=35 \%)$. A common pathway model was then estimated to examine the ACE contributions of these domains to a latent resilience factor. Variance in latent resilience was predominantly explained by $\mathrm{A}$ and $\mathrm{E}(\mathrm{A}=$ $50 \%, \mathrm{C}=15 \%, \mathrm{E}=35 \%$ ), although only $\mathrm{E}$ was statistically significant (follow up Monte-Carlo analyses suggest power was too low to detect statistically significant contributions). These results suggest that although different domains of resilience are differentially heritable, there is evidence of at least moderate genetic influence on resilience of neighborhood disadvantage.

References
Vanderbilt-Adriance, E., \& Shaw, D. S. (2008). Conceptualizing and re-evaluating resilience across levels of risk, time, and domains of competence. Clinical Child and Family Psychology Review, 11(1-2), $30-58$.

\section{Using neuroticism as a model phenotype to compare and contrast genome-wide associations from structural equation models}

Brad Verhulst ${ }^{1}$, John Hettema ${ }^{1}$

${ }^{1}$ Department of Psychiatry and Behavioral Health, Texas A\&M University, College Station, Texas, USA

\section{Keywords Neuroticism, GWAS, GW-SEM, genomicSEM, TATES}

Several methods have emerged to conduct genome wide association studies using structural equation modeling (SEM). We will use the moderately heritable personality trait Neuroticism as a model phenotype to compare and contrast the SEM methods, focusing on potential differential interpretations to elucidate the strengths and weaknesses of each method. Specifically, we will compare GW-SEM (Verhulst et al. 2017), genomicSEM (Grotzinger et al. 2018), and TATES (van der Sluis et al. 2013).

Participants: Analyses will be conducted on up to 379,153 unrelated respondents with northern European ancestry from the UK Biobank.

Statistical Analyses: We will conduct univariate GWAS analyses on the 12 Neuroticism items and use the summary statistics for the genomicSEM and TATES analyses. We will compare these results with those using raw data conducted with GW-SEM.

We expect the pattern of genetic associations from different software will be broadly consistent, however possible differences may emerge based on methodological variation. These potential methodological differences are not currently understood but may have considerable implications for the utility of the specific methods.

\section{References}

Verhulst, et al., (2017). GW-SEM: A statistical package to conduct genome-wide structural equation modeling. Beh Gen, 47(3), 345-359.

Grotzinger, et al. (2019). Genomic structural equation modelling provides insights into the multivariate genetic architecture of complex traits. Nat Hum Behav, 3(5), 513-525.

van der Sluis et al (2013) TATES: Efficient Multivariate Genotype-Phenotype Analysis for Genome-Wide Association Studies. PLOS GEN.

Grant Support: R01 MH113665

\section{Exploring genetic relationships between musicality and social behavior}

Janne R Vermeulen ${ }^{1,4}$, Fenja Schlag ${ }^{1}$, Ellen Verhoef ${ }^{1}$, Celeste Figaroa $^{1}$, Jeffrey van der Ven ${ }^{1,4}$, Simon E Fisher ${ }^{1,2}$, Beate St Pourcain ${ }^{1,2,3}$

${ }^{1}$ Language and Genetics, MPI for Psycholinguistics, Nijmegen, The Netherlands

${ }^{2}$ Donders Institute for Brain, Cognition and Behaviour, Radboud University, Nijmegen, The Netherlands

${ }^{3}$ Bristol Medical School, University of Bristol, Bristol, UK

${ }^{4}$ Master Medical Biology, Radboud University, Nijmegen, The Netherlands 
Keywords Genetic Correlations, Structural Equation Modelling, Musicality, Social Behavior

The ability to produce and perceive music (musicality) plays a significant role in all human societies and the participation in musical events promotes friendships, emotional sensitivity and social skills. Musicality and social behavior are complex heritable phenotypes, although their shared genetic aetiology is not well understood. Here, we investigate (i) evidence for genetic overlap between musicality and social behavior and (ii) whether genetic relationships remain stable during the course of development. Analyses were conducted in unrelated children from the ALSPAC-cohort. We examined two musicality-related parent reports (at 5 and 6 years, $51.3 \%$ males, $\mathrm{N} \leq$ $5881)$ where at least $1 \%$ of children reported problems $(\mathrm{N}=232$ (3.9\%) and $\mathrm{N}=136(1.7 \%)$, respectively), two longitudinal social traits, low prosociality and peer problems (Rutter parent scale and Strengths and Difficulties Questionnaire, 4 to 17 years, $50.2 \%$ males, $\mathrm{N} \leq 6135$ ), as well as genome-wide data. SNP-heritability and genetic correlations were assessed with genome-wide complex trait analysis (GCTA) and the genetic stability of social phenotypes during development explored using genetic-relationship-matrix structural equation modelling (GSEM). The multiple testing threshold was 0.0125; SNPheritability estimates ranged from $3 \%$ ( $\mathrm{SE}=0.06$ ) to $37 \%$ ( $\mathrm{SE}=$ 0.09). Our results showed that problems in musical abilities (e.g. 'Child cannot hum a tune' at 5 years) were positively correlated with mother-reported peer problems across mid-childhood (at 7 years: GCTA- $r_{g}=1.00(\mathrm{SE}=0.50), \mathrm{p}=0.004$; at 10 years: GCTA- $\mathrm{r}_{\mathrm{g}}=0.75$ $(\mathrm{SE}=0.34), \mathrm{p}=0.005)$. Longitudinal GSEM analysis of peer problems identified evidence for a shared genetic factor that remains stable during development. Thus, children with more developed musical abilities in early childhood may have consistently better peer relationships in later life.

\section{Testing for moderation of mid-life cognitive performance by sleep duration}

Tina Vo ${ }^{1}$, Shandell Pahlen ${ }^{1}$, William Kremen ${ }^{2}$, Matt Mc Gue ${ }^{3}$, Chandra A. Reynolds ${ }^{1}$

${ }^{1}$ Department of Psychology, University of California, Riverside, Riverside, California, USA ${ }^{2}$ Department of Psychiatry, University of California, San Diego, San Diego, California, USA ${ }^{3}$ Department of Psychology, University of Minnesota, Minneapolis, Minnesota, USA

Keywords Sleep, Cognitive Aging, Sleep Duration, Cognition, Moderation

Sleep duration is associated with cognition at midlife, a key period predictive of late-life cognitive aging, however whether sleep duration moderates the etiology of cognitive performance is unclear. We performed initial analyses using an informative twin design, drawing from Minnesota Twin Study of Adult Development and Aging $\left(\right.$ MTSADA; $X_{\text {age }}=58.7, \mathrm{~N}=918, \mathrm{~F}=59.5 \%, 540 \mathrm{MZ}$ individuals, $378 \mathrm{DZ}$ individuals) one of the participating studies within the Interplay of Genes and Environments across Multiple Studies (IGEMS) consortium. Measures for this study included sleep duration and three cognitive functioning measures (Digit Symbol, Vocabulary, and Block Design subtests). All analyses included adjustments for age and sex. Phenotypically, the correlations between sleep duration and cognitive performance were modest $(r=.03-.08)$. Twin correlations for sleep duration, controlling for age and sex, were $r \mathrm{MZ}=.36, r \mathrm{DZ}=$ .27 indicating substantial environmental influence. Univariate ACE moderation models were fitted accounting for both self and co-twin sleep duration to evaluate moderation of variability in cognitive performance by sleep duration. Age moderation was simultaneously fitted. Full models suggested that genetic variance in cognition decreased as sleep duration increased on the cognitive task of block design $\left(a_{5 \mathrm{hrs}}^{2}=.50, a_{9 \mathrm{hrs}}^{2}=.04\right)$, while remaining fairly stable for digit symbol $\left(a_{5 \mathrm{hrs}}^{2}=.21, a_{9 \mathrm{hrs}}^{2}=.23\right)$ and vocabulary $\left(a_{5 \mathrm{hrs}}^{2}=.58, a_{9 \mathrm{hrs}}^{2}=\right.$ .33). Shared common environment contributions $\left(\mathrm{c}^{2}\right)$ in cognition increased as sleep duration increased on all cognitive tasks $\left(c_{5 \mathrm{hrs}}^{2}=\right.$ $\left..14-.25, c_{9 \text { hrs }}^{2}=.43-.58\right)$. Patterns of unique environmental contributions $\left(\mathrm{e}^{2}\right)$ differed by cognitive task. These preliminary findings may add to the growing behavioral genetic literature, possibly indicating that sleep may moderate health related outcomes including cognitive functioning at midlife.

Grant Support: R01 AG059329, R01 AG060470, R0I AG0688

\section{Genetic and environmental influences on non-ability- based confidence}

Randi L. Vogt ${ }^{1}$, Anqing Zheng ${ }^{1}$, Daniel A. Briley ${ }^{1}$, Margherita Malanchini $^{2}$, K. Paige Harden ${ }^{3}$, and Elliot M. Tucker-Drob ${ }^{3}$

${ }^{1}$ Department of Psychology, University of Illinois at Urbana-Champaign, Champaign, Illinois, USA

${ }^{2}$ Department of Psychology, Queen Mary University of London, London, UK

${ }^{3}$ Department of Psychology and Population Research Center, University of Texas at Austin, Austin, Texas, USA

Keywords Non-Ability-Based Confidence, Overconfidence, Self-Perception, Personality, Cognitive Ability

Non-ability-based confidence is the extent to which an individual's confidence is not calibrated to their ability. Many people misperceive their abilities in some area of their lives and have more or less confidence than their abilities reflect, leading to differences in competitiveness, persistence, and perceived prestige. Despite the ubiquity and consequential nature of non-ability-based confidence, there is relatively little research on its correlates. In this study, we use the Texas Twin Project, a cross-sectional, genetically informed dataset of 3rd to 8 th grade children $(N=1588$ individuals, $N=814$ sibling pairs) to investigate non-ability-based confidence. Non-ability-based confidence was assessed using self-reported intellectual self-concept and standardized measures of crystalized intelligence, fluid intelligence, and processing speed. First, we find that non-ability-based confidence is strongly positively correlated with need for cognition, mastery goal orientation, grit, openness to experiences, and emotional stability. Then, we decompose non-ability-based confidence into its genetic and environmental components and find that it is $15-28 \%$ heritable with the remaining variance primarily due to the nonshared environment. Finally, we find that genetic factors account for approximately $59 \%$ of the association with other personality measures. Non-ability-based confidence is present in early childhood and shares developmental inputs with a variety of psychological characteristics due to both genetic and environmental processes.

Grant Support: Daniel A. Briley and Elliot M. Tucker-Drob were supported by Jacobs Foundation Research Fellowships. Tucker-Drob and Harden are Faculty Research Associates of the Population Research Center at the University of Texas at Austin, which is supported by NIH grant P2CHD042849. Data collection for the Texas Twin Project has been supported by NIH grants R01HD083613, R01HD092548, R21HD081437, R21AA023322, and R21AA020588. 


\section{The impact of genetic nurture on educational attainment: a meta-analysis}

Biyao Wang ${ }^{1}$, Jessie Baldwin ${ }^{1}$, Tabea Schoeler ${ }^{1}$, Jean-Baptiste Pingault ${ }^{1,2}$

${ }^{1}$ Division of Psychology and Language Sciences, University College London, London, United Kingdom

${ }^{2}$ Social, Genetic and Developmental Psychiatry Centre, Institute of Psychiatry, Psychology \& Neuroscience, King's College London, London, United Kingdom

Keywords Educational Attainment; genetic nurture; polygenic score; family-based study; meta-analysis

Research on the intergenerational transmission of complex traits such as Educational Attainment (EA) is often confounded by the mixture of genetic effects and environmental effects as parents both transmit genes and create environments for their offspring. The phenomenon of parental non-transmitted alleles affecting offspring outcomes is called "genetic nurture" (Kong et al. 2018). Genotyped families of parent-child pairs or mother-father-child trios allow us to identify the effect of genetic nurture by testing whether parents' genetics predict child outcomes over and above children's genetics. Here, we conducted the first meta-analysis to quantify the effect of genetic nurture on EA. Multilevel random-effects models and meta-regression were employed to (a) estimate the associations between parental genotype and child genotype with children's EA, (b) estimate the effect of genetic nurture on the associations between genotype and EA. We identified 52 estimates from 9 studies of genotyped families $(\mathrm{N}=$ 19,316). We found that child EA was predicted by both parental genotype (beta $=0.20,95 \%$ CI $[0.16,0.25]$ ) and child genotype (beta $=0.24,95 \%$ CI $[0.20,0.28])$. Genetic nurture had a small effect on child EA, as parental genotype predicted child EA over and above child genotype $\left(\right.$ beta $_{\text {adjusted }}=0.08,95 \%$ CI $[0.06,0.10]$ ), while the effect of child genotype attenuated after controlling for genetic nurture $\left(\right.$ beta $_{\text {adjusted }}=0.15,95 \%$ CI $\left.[0.11,0.20]\right)$. These results demonstrate a consistent effect of genetic nurture on child EA across studies and highlight the impact of intergenerational transmission through environmental pathways.

\section{Reference}

Kong et al. (2018). The nature of nurture: Effects of parental genotypes. Science, 359(6374), 424-428.

\section{Using genetic correlations and polygenic risk prediction to analyze shared genetics between sleep deficits and cannabis use behaviors}

Evan A. Winiger ${ }^{1,2}$, Jarrod M. Ellingson ${ }^{1,3}$, Claire L. Morrison ${ }^{1,2}$, Robin P. Corley ${ }^{1}$, Joëlle A. Pasman ${ }^{4}$, Tamara L. Wall ${ }^{5}$, Christian J. Hopfer $^{3}$, John K. Hewitt ${ }^{1,2}$

${ }^{1}$ Institute for Behavioral Genetics, University of Colorado Boulder

${ }^{2}$ Department of Psychology and Neuroscience, University of Colorado Boulder

${ }^{3}$ Department of Psychiatry, University of Colorado School of Medicine

${ }^{4}$ Behavioural Science Institute, Radboud University Nijmegen, The Netherlands

${ }^{5}$ Department of Psychiatry, University of California, San Diego, CA

Keywords Cannabis, Cannabis Use Disorder, Sleep Duration, Insomnia, Chronotype, Genetics
Increased cannabis frequency is associated with shorter sleep duration, increased rates of insomnia, and an eveningness chronotype. It is thought that the endocannabinoid system is involved in the regulation of sleep processes and that various sleep and circadian rhythm related genes are associated with cannabis use. We used summary statistics from the most recent European derived sleep and cannabis behavior GWAS to analyze genetic correlations between the domains of cannabis use and sleep deficits using Linkage Disequilibrium Score Regression (LDSC). We also generated polygenic risk scores (PRS) of sleep deficits to predict cannabis use traits in a self-identifying allwhite target sample consisting of high-risk participants and participants from twin/family community-based studies $(\mathrm{n}=796$, male $=$ $66 \%$; mean age $=26.81$ ). Using LDSC, we found significant genetic correlations between lifetime cannabis use and an eveningness chronotype $(\mathrm{rG}=0.24)$ as well as genetic correlations between cannabis use disorder and both short sleep duration $(\mathrm{rG}=0.23)$ and insomnia $(\mathrm{rG}=0.20)$. Controlling for sex, age, ancestral principal components (PCs 1-10), current depression symptoms, and past 180-day alcohol and tobacco use, an insomnia PRS predicted earlier age of first cannabis use $(\beta=-0.09, p=0.02)$ and increased lifetime CUD symptom count $(\beta=0.07, p=0.03$ ). Ultimately, we found evidence of shared genetics between sleep deficits and increased cannabis use behaviors, implying shared genetic risk and liability between these domains.

Grant Support: DA035804, DA032555, DA042755, DA017637, and DA011015.

\section{Association of family history of schizophrenia with clinical features in eating disorders}

Ruyue Zhang ${ }^{1}$, Ralf Kuja-Halkola ${ }^{1}$, Cynthia M. Bulik ${ }^{1,2,3}$, Sarah E. Bergen $^{1}$

${ }^{1}$ Department of Medical Epidemiology and Biostatistics, Karolinska Institutet, Stockholm, Sweden

${ }^{2}$ Department of Psychiatry, University of North Carolina School of Medicine, Chapel Hill, NC, USA

${ }^{3}$ Department of Nutrition, University of North Carolina at Chapel Hill, Chapel Hill, NC, USA.

Keywords Schizophrenia, Family History, Eating Disorders, Clinical Features.

Large-scale family studies on the co-aggregation of eating disorders (EDs) and schizophrenia have revealed shared genetic and environment factors between these conditions. However, the etiological and clinical relationships between schizophrenia and EDs remain unclear. To determine whether a family history of schizophrenia influences the clinical course of EDs, we conducted a cohort evaluation of family histories of schizophrenia in individuals born in Sweden between 1977 and 2003 who have ever received diagnoses for anorexia nervosa (AN) or other EDs (OED: bulimia nervosa, binge-eating disorder, and EDs not otherwise specified). In individuals with OED, the presence of schizophrenia in first degree relatives was associated with an increased risk for comorbid anxiety (hazard ratio[HR] $=1.36$; $95 \% \mathrm{CI}=1.11-1.67)$ and obsessive-compulsive disorder $(\mathrm{HR}=1.59$; $95 \% \mathrm{CI}=1.08-2.36)$. Similarly, in individuals with AN, schizophrenia in first degree relatives was associated with an increased risk for anxiety $(\mathrm{HR}=1.47 ; 95 \% \mathrm{CI}=1.08-2.01)$ but not OCD $(\mathrm{HR}=1.01 ; 95 \% \mathrm{CI}=0.51-2.01)$. Individuals with OED who have first degree relatives with schizophrenia tend to receive more total diagnoses (incidence rate ratio $[$ IRR] $=1.26 ; 95 \% \mathrm{CI}=$ $1.07-1.48)$ and unique diagnoses $(\mathrm{IRR}=1.24 ; 95 \% \mathrm{CI}=1.08-1.41)$ and have more suicide attempts (IRR $=1.70 ; 95 \% \mathrm{CI}=1.05-2.76)$. However, this was not observed in individuals with AN. Having any 
first-, second-, or third degree relative with schizophrenia resulted in a similar pattern of associations but of lesser magnitude compared with having schizophrenia in first degree relatives. These findings may provide new insights into the different manifestations of EDs and potentially influence treatment for individuals with first degree relatives with schizophrenia. Further analyses will explore whether molecular genetic risk for schizophrenia influences ED course.

Grant Support: Chinese Scholarship Council (CSC201700260258), Swedish Medical Association \#777621 (PI: SB), Swedish Research Council: 538-2013-8864 (PI: CB)

\section{Identification and characterization of common genetic risk factors for chronic pain and psychopathology}

Katerina Zorina-Lichtenwalter ${ }^{1,2}$, Marta Čeko $^{2}$, Subrata Paul ${ }^{1}$, Naomi P Friedman $^{1}$, Matthew C. Keller ${ }^{1}$, Tor D. Wager ${ }^{2,3}$

${ }^{1}$ Department of Psychology and Neuroscience and Institute for Behavioral Genetics, University of Colorado Boulder, Boulder, CO, USA

${ }^{2}$ Department of Psychology and Neuroscience and Institute of Cognitive Science, University of Colorado Boulder, Boulder, CO, USA ${ }^{3}$ Department of Psychological and Brain Sciences, Dartmouth College, Hanover, NH, USA

Keywords Chronic Pain, Psychological Conditions, GWAS, Genomic SEM

Chronic pain patients often suffer from multiple pain disorders and other forms of psychopathology (particularly anxiety and depression).
Changes in overlapping brain pathways and response to the same therapeutic interventions suggest shared psychological and physiological risk factors for multiple psychiatric and neurological disorders. A number of studies have likewise reported significant genetic correlations between chronic pain and several forms of psychopathology. Nevertheless, the underlying genetic architecture and specific risk variants are still largely unknown. Here, we aim to elucidate the genetic landscape and characterize molecular pathways that lie at the intersection of these disorders. Our analyses are organized into 4 stages: (1) A genome-wide association study (GWAS) on all available pain and psychological conditions in the UK Biobank cohort; (2) Phenotype factor analysis and genomic structural equation modeling (genomicSEM) using association statistics from stage 1; (3) GWAS on factors extracted from stage 2 to identify single nucleotide polymorphisms (SNPs) associated with genetic predisposition to multiple forms of chronic pain and psychopathology; and ((4) Gene enrichment and pathway analysis of associated SNPs. We hope that our findings will reveal (a) whether one or more common factors underlie chronic pain and psychopathology; (b) whether these common factors are related to specific pain disorders and psychiatric diagnoses; (c) whether causes of pain related to brain and psychological health can be dissociated from those related to peripheral injury and tissue characteristics; and (d) genetic markers for nonspecific and tissue-specific risk factors for chronic pain, to be used for pain prognosis and counselling of at-risk patients.

Grant Support: NIDA R01 DA046064 (Friedman, Wager).

Publisher's Note Springer Nature remains neutral with regard to jurisdictional claims in published maps and institutional affiliations. 